\title{
Two decades of DNA barcoding in the genus Usnea (Parmeliaceae): how useful and reliable is the ITS?
}

\author{
Robert Lücking ${ }^{1 *}$, Miko Rain Abraham Nadel${ }^{2}$, Elena Araujo 3 \& Alice Gerlach ${ }^{4}$
}

\section{Article info}

Received: 7 Aug. 2020

Revision received: 11 Nov. 2020

Accepted: 11 Nov. 2020

Published: 29 Dec. 2020

\section{Associate Editor}

María de los Angeles Herrera Campos

\begin{abstract}
We present an exhaustive analysis of the ITS barcoding marker in the genus Usnea s.lat., separated into Dolichousnea, Eumitria, and Usnea including the subgenus Neuropogon, analyzing 1,751 accessions. We found only a few low-quality accessions, whereas information on voucher specimens and accuracy and precision of identifications was of subpar quality for many accessions. We provide an updated voucher table, alignment and phylogenetic tree to facilitate DNA barcoding of Usnea, either locally or through curated databases such as UNITE. Taxonomic and geographic coverage was moderate: while Dolichousnea and subgenus Neuropogon were well-represented among ITS data, sampling for Eumitria and Usnea s.str. was sparse and biased towards certain lineages and geographic regions, such as Antarctica, Europe, and South America. North America, Africa, Asia and Oceania were undersampled. A peculiar situation arose with New Zealand, represented by a large amount of ITS accessions from across both major islands, but most of them left unidentified. The species pair Usnea antarctica vs. U. aurantiacoatra was the most sampled clade, including numerous ITS accessions from taxonomic and ecological studies. However, published analyses of highly resolved microsatellite and RADseq markers showed that ITS was not able to properly resolve the two species present in this complex. While lack of resolution appears to be an issue with ITS in recently evolving species complexes, we did not find evidence for gene duplication (paralogs) or hybridization for this marker. Comparison with other markers demonstrated that particularly IGS and RPBI are useful to complement ITS-based phylogenies. Both IGS and RPBI provided better backbone resolution and support than ITS; while IGS also showed better resolution and support at species level, $R P B 1$ was less resolved and delineated for larger species complexes. The nuLSU was of limited use, providing neither resolution nor backbone support. The other three commonly employed protein-coding markers, TUB2, RPB2, and MCM7, showed variable evidence of possible gene duplication and paralog formation, particularly in the $M C M 7$, and these markers should be used with care, especially in multimarker coalescence approaches. A substantial challenge was provided by difficult morphospecies that did not form coherent clades with ITS or other markers, suggesting various levels of cryptic speciation, the most notorious example being the $U$. cornuta complex. In these cases, the available data suggest that multimarker approaches using ITS, IGS and RPBI help to assess distinct lineages. Overall, ITS was found to be a good first approximation to assess species delimitation and recognition in Usnea s.lat., as long as the data are carefully analyzed, and reference sequences are critically assessed and not taken at face value. In difficult groups, we recommend IGS as a secondary barcode marker, with the option to employ more resource-intensive approaches, such as RADseq, in species complexes involving so-called species pairs or other cases of disparate morphology not reflected in the ITS or IGS. Attempts should be made to close taxonomic and geographic gaps especially for the latter two markers, in particular in Eumitria and Usnea s.str. and in the highly diverse areas of North America and Central America, Africa, Asia, and Oceania.
\end{abstract}

Key words: accuracy in GenBank, incorrect sequence labeling, paralogs, species delimitation; species richness

\footnotetext{
${ }^{1}$ Botanischer Garten und Botanisches Museum, Freie Universität Berlin, Königin-Luise-Straße 6-8, 14195 Berlin, Germany

2 San Francisco State University, Department of Biology, 1600 Holloway Ave., San Francisco, CA 94132, USA

3 Departamento de Biología Vegetal II, Facultad de Farmacia, Universidad Complutense de Madrid, 28040 Madrid, Spain

${ }^{4}$ Conservatoire et Jardin botaniques de la Ville de Genève, $1 \mathrm{ch}$. de l'Impératrice, 1292 Chambésy/GE, Switzerland

* Corresponding author e-mail: r.luecking@bgbm.org
} 


\section{Introduction}

DNA barcoding refers to the use of a particular marker to identify species relative to a set of identified reference sequences. DNA-based delimitation and identification of fungi was discussed already in the mid-1980s (Kurtzman 1985), and molecular phylogenetic work on fungi including lichens took off in the 1990s (Bruns et al. 1991; Gargas \& Taylor 1995; Gargas et al. 1995). The internal transcribed spacer (ITS) of the ribosomal DNA cistron was one of the first markers used to reconstruct fungal phylogenies (White et al. 1990; Lee \& Taylor 1992; Rehner \& Uecker 1994; Feibelman et al. 1994; Kusaba \& Tsuge 1995; Goffinet \& Bayer 1997), including the pioneering work on lichen photosymbiodemes by Daniele Armaleo and Philippe Clerc (Armaleo \& Clerc 1991). Molecular identification of fungi including lichens using the ITS also emerged in the early 1990s (Kasuga et al. 1993; Tisserat et al. 1994; Groner \& LaGreca 1997; Lohtander et al. 1998), and ITS was employed for population genetics in conservation assessments in the model taxon Lobaria pulmonaria (L.) Hoffm. (Zoller et al. 1999). However, only less than a decade ago, the ITS was proposed as the universal DNA barcoding marker for fungi (Schoch et al. 2012).

Usnea Dill. ex Adans. is one of the largest genuslevel clades of lichenized fungi, currently listed among the ten most speciose genera, with approximately 350 taxa (Thell et al. 2012; Lücking et al. 2017a). The classification of usneoid lichens has been in flux, besides Protousnea (Motyka) Krog either recognizing a single genus Usnea s.lat., with several subgenera and sections (Motyka 1936, 1938; Ohmura 2002; Ohmura \& Kanda 2004; Wirtz et al. 2006; Truong \& Clerc 2013; Ohmura \& Kashiwadani 2018; Temu et al. 2019) or separating Dolichousnea (Y. Ohmura) Articus, Eumitria Stirt., and Neuropogon Nees \& Flot. (Fig. 1), as smaller genera from Usnea s.str. (Articus 2004). Given the underlying data, we follow Divakar et al. (2017) and Kraichak et al. (2017) to recognize Dolichousnea and Eumitria as separate genera, while retaining Neuropogon within Usnea at the level of subgenus. Besides considerations of evolutionary age, this reflects the fact that both Dolichousnea and Eumitria have consistently been recovered as monophyletic groups in all phylogenetic studies, whereas neuropogonoid taxa are polyphyletic and the neuropogonoid core group was mostly found nested within Usnea s.str., rendering the latter paraphyletic (Ohmura 2002; Articus 2004; Ohmura $\&$ Kanda 2004; Wirtz et al. 2006). The only study rendering Neuropogon s.str. and Usnea s.str. reciprocally monophyletic, based on six markers, had a limited taxon sampling (Divakar et al. 2017; Kraichak et al. 2017). Since neuropogonoid taxa such as $U$. acanthella (I.M. Lamb) F.J. Walker and U. durietzii Motyka are nested within Usnea s.str. (Wirtz et al. 2006), there are also no clearcut morphological characters to support Neuropogon as a separate genus. Rather, the production of melanoid substances in the thalline cortex and the apothecial discs has been considered an adaptation to harsh environmental conditions, as neuropogonoid taxa are typically found in habitats with high UV radiation (Lumbsch \& Wirtz 2011). Likewise, the phenotypic distinction of Dolichousnea and Eumitria from Usnea s.str. is not straightforward (see below).

Species identification in Usnea s.lat. is notoriously difficult. Usnea was therefore among the first genera where DNA-based identification via the ITS was tested. The first ITS sequence generated in this genus was for a specimen of $U$. florida (L.) F.H. Wigg from Sweden (AF117996; Mattson 4001, UPS), although the sequence was not published at the time because it was considered '... too preliminary to be submitted to GenBank ...' (Mattson \& Wedin 1998: 465). It was published one year later (Wedin et al. 1999), thus losing the race for the first ITS sequence of Usnea registered with GenBank to U. strigosa (Ach.) Pers. (AF112990), used as outgroup in a study on Ramalina (LaGreca 1999).

Shortly after the turn of the millennium, the first studies emerged that used the ITS to assess species delimitation in Usnea s.lat. Initially, focus was laid on the taxonomic status of so-called species pairs, forms with identical morphology, but differing reproductive strategy, such as the apotheciate $U$. florida vs. the sorediate U. subfloridana Stirt. (Articus et al. 2002). At the time, the authors concluded that the two morphs represented a single species. Another objective was the delimitation of natural groupings within Usnea s.lat. based on the ITS (Ohmura 2002; Articus 2004; Ohmura \& Kanda 2004; Wirtz et al. 2006). Based on Japanese taxa, Ohmura (2002) resolved Dolichousnea as sister to a clade with Eumitria sister Usnea s.str., recognizing the three clades at subgenus level. Articus (2004) recovered Dolichousnea plus Eumitria as sister to Neuropogon plus Usnea and proposed to recognize the four groups at genus level, although both ITS alone and a three-locus dataset (ITS, nuLSU, $\beta$-tubulin $=T U B 2)$ rendered Usnea s.str. paraphyletic with respect to Neuropogon. Ohmura \& Kanda (2004), again based on ITS, recovered Eumitria as sister to the other three groups and Dolichousnea as sister to Neuropogon plus Usnea, also with the latter forming a paraphyletic grade relative to Neuropogon. Wirtz et al. (2006) found an ITS-based topology similar to that of Articus (2004), with Neuropogon polyphyletic and nested within Usnea s.str., and proposed to recognize a single, large genus Usnea s.lat., also subsuming Dolichousnea and Eumitria within the latter.

Most subsequent works utilizing the ITS, without or with additional markers, focused on species taxonomy, with emphasis on neuropogonoid taxa (Seymour et al. 2007; Wirtz et al. 2008, 2012; Lumbsch \& Wirtz 2011). Several studies worked on other groups within Usnea s.lat., such as Eumitria (Temu et al. 2019), Usnea s.str. with pigmented cortex (Ohmura 2008), and the U. cornuta Körb. aggregate (Gerlach et al. 2019a). Geographic focal studies included Usnea from North America and Europe (Mark et al. 2016a; Clerc \& Naciri 2021), European shrubby species (Saag et al. 2011), the Iberian Peninsula (Araujo 2016), Taiwan (Shen et al. 2012), Africa (Nadel 2016), South America (Truong et al. 2013a; Truong \& Clerc 2016; Gerlach et al. 2017, 

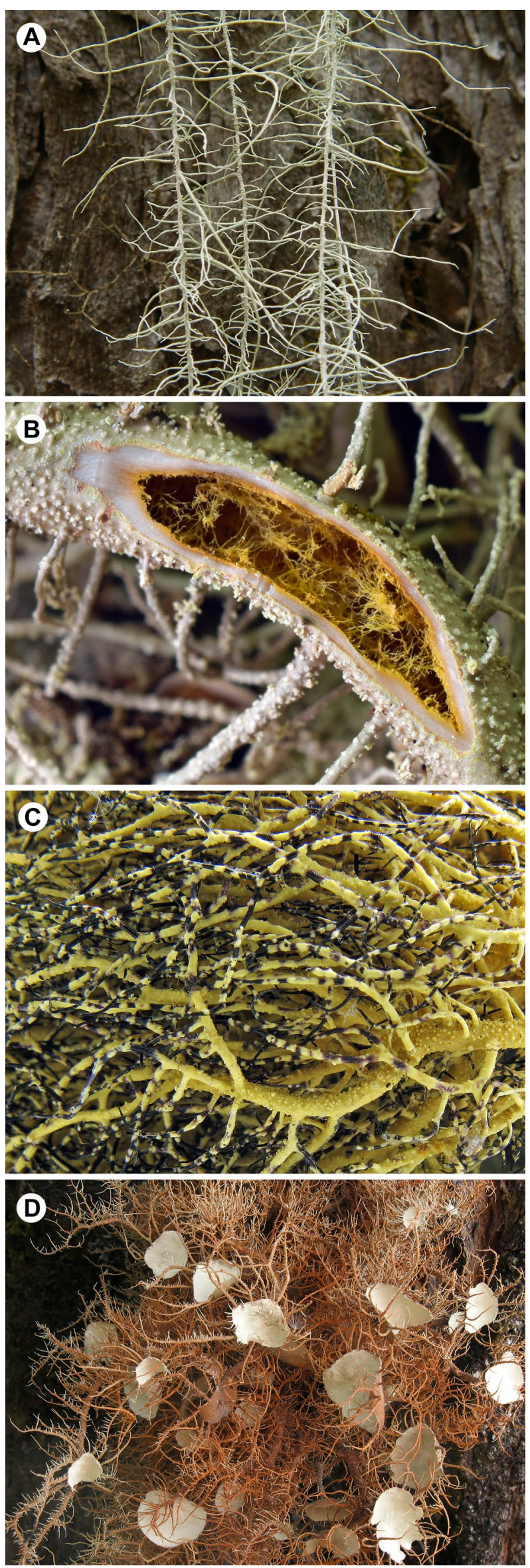

Figure 1. Representatives of the four main groups of Usnea s.lat. A Dolichousnea (D. longissima); B - Eumitria (E. baileyi); C - subgenus Neuropogon (U. sphacelata); D - Usnea s.str. (U. erinacea). Photographs by RL except (A), kindly provided by Y. Ohmura. 2019a, b), and urban New York (Dorey et al. 2019). The thesis works by E. Araujo (Araujo 2016) and M. Nadel (Nadel 2016) have not been published and the underlying sequences have not been deposited in GenBank previously, but are included in the present study with newly issued accession numbers.

Broader DNA barcoding studies also included data on Usnea or in part focused on the latter, particularly in New Zealand (Kelly et al. 2011), but also Switzerland (Mark et al. 2016b), Norway (Marthinsen et al. 2019), and Cameroon (Orock et al. 2012). A large amount of ITS data was generated for analyses of mycobiont-photobiont associations of Usnea lichens and their corresponding ecology and morphology in New Zealand and Antarctica (Rafat 2014; Buckley et al. 2014; Cao et al. 2015, 2018; Park et al. 2015; Rafat et al. 2015). Another broad study in the Northern Hemisphere focused on genetic variation in Dolichousnea longissima (Ach.) Articus (Rolstad et al. 2013). These data were subsequently used to employ niche distribution modeling to predict geographic outliers not representing that species using a novel approach to visualize ecogeographic barriers (Smith et al. 2016). Millanes et al. (2014) analyzed relationships between lichenicolous Biatoropsis Räsanen species and their Usnea hosts. The level of resolution of the ITS was tested in two studies on the species pair $U$. antarctica Du Rietz and U. aurantiacoatra (Jacq.) Bory using newly developed microsatellite markers (Lagostina et al. 2018) and RADseq (Grewe et al. 2018). Both studies supported the two taxa as separate species, although they were not resolved by ITS.

Using DNA sequences for the identification of fungi has been criticized early on (Seifert et al. 1995), but has since become established as an indispensable component of integrative taxonomy of fungi (Lücking et al. 2020a). However, the application of ITS as a universal fungal barcoding marker (Schoch et al. 2012) has been challenged by lack of resolution in certain fungal lineages and by potential artifacts due to intragenomic variation reflecting either hybridization and introgression or gene duplication (Nilsson et al. 2008; Linder \& Banik 2011; Pino-Bodas et al. 2013; Stielow et al. 2015; Li et al. 2017; Badotti et al. 2018; Lücking et al. 2020a). Another notorious problem pertains to data quality, both the quality of the underlying sequence data and the quality of metadata, in particular sequence identifications (Nilsson et al. 2006, 2018; Bidartondo 2008; Kozlov et al. 2016; Schoch et al. 2017; Meiklejohn et al. 2019; Lücking et al. 2020a, b; Clerc \& Naciri 2021). Here, we use the example of the genus Usnea s.lat. to quantitatively assess these issues, by analyzing all 1,610 ITS sequences deposited under the label Usnea in GenBank between 1999 and 2020 (cut-off date: 15 June 2020), plus the 147 previously unregistered sequences from Araujo (2016) and Nadel (2016). We want to emphasize that this work is not to be understood as a phylogenetic revision of Usnea s.lat. The latter would require careful examination of all underlying vouchers in the context of the historic taxonomy of this genus. Rather, we attempted to establish a vetted phylogenetic framework for DNA barcoding approaches, given the data at hand, which are still far from complete. 
We dedicate this work to our esteemed colleague, mentor and friend, Philippe Clerc, on the occasion of his retirement from active duty as Head of the Herbarium at the Conservatoire et Jardin Botaniques de la Ville de Genève. Since his first papers on the topic 36 years ago (Clerc 1984a, b), Philippe has been instrumental in shaping the taxonomy of Usnea s.lat., one of the largest and taxonomically most difficult genera of lichenized fungi, through his own, numerous contributions, through fruitful international collaborations, and through training of various generations of usneologists around the world. In these modern times, where taxonomy is moving towards a misguided concept of automatism, Philippe's work demonstrates the requirement for individual dedication and the motivation to learn, gain experience, and transfer this knowledge, to achieve long-lasting impact in taxonomy and in cataloguing our planet's biodiversity. We wish Philippe our best for his future endeavors, which hopefully will include a good deal of 'usneology'. We humbly recognize that he is the one person missing as expert co-author in this study dedicated to him, and we hope he will forgive the errors we may have made in trying to make sense of our findings in this complex genus.

\section{Material and methods}

\section{Species concept}

The present study focuses on molecular data used to formulate species hypotheses. As far as phenotype data (including secondary chemistry) were available, we integrated these into the assessment of species delimitations on an ad hoc basis in the discussions under each clade. However, our primary approach was to delimit clades using a combination of branch support and branch length. We thereby established phylogenetic species hypotheses using either reciprocal monophyly or, in exceptional cases, nested relationships leading to the recognition of paraphyletic species. Paraphyletic species are a reality of evolution (Crisp \& Chandler 1996; Funk \& Omland 2003; Kuchta et al. 2018) and we accepted such instances under the following conditions: (1) the nested clade had a substantially longer stem branch than other terminal branches in the including clade; (2) the stem branch of the nested clade was strongly supported; (3) phenotypic and/or geographic differences were associated with the nested clade $v s$. its paraphyletic residual.

We should also emphasize that while accurate species hypotheses are critical to the DNA barcoding approach, the performance of barcoding markers is first and foremost lineage-based, regardless at what taxonomic level these lineages are recognized.

\section{Molecular data analyses}

Data assembly. We downloaded all available sequence data for the genus Usnea from GenBank and added unpublished sequences from the thesis works of Araujo (2016) and Nadel (2016). After separating sequences that combined several markers (e.g., nuSSU, ITS,
nuLSU), the data set was automatically aligned using MAFFT 7.244 with the [--auto] and the [--reorder] function (Katoh \& Standley 2013). The entire block of ITS sequences (1,757 total) was extracted from this data set, automatically realigned again in MAFFT, and manually inspected and adjusted in BioEdit 7.2.5 (Hall 1999, 2011). The alignment was subsequently refined by first removing ambiguously aligned regions, reconstructing a tree, and then resorting the original sequences based on the tree so that more variable regions could be directly compared between closely related species. Sequence names were then batch-edited to reflect the format $>$ Genus_species_Accession_Country_sampleID, by saving the FASTA file as a text file, formatting it for manipulation in MICROSOFT Excel 10, extracting the names block and filtering and editing the corresponding name elements. Three sequences of Protousnea dusenii were used as outgroup.

After reconstructing a maximum likelihood tree (see below), the sequences were resorted following their arrangement in the tree. This was accomplished by extracting the names in corresponding order from the PDF-format tree file, opening them in MICROSOFT Excel 10 and numbering them consecutively. The underlying FASTA alignment was also opened in MICROSOFT Excel 10 after preformatting in MICROSOFT Word 10 to achieve placement of elements in separate columns, and the sequences were resorted using the numbers from the tree file. After this step, which sorted sequences according to their phylogenetic relationships instead of similarity, the alignment was again manually inspected and adjusted. This process was repeated three times to obtain the final alignment with a length of 636 bases (File S1). The sequence metadata were then edited in table form in MICROSOFT Excel 10 and the corresponding original publications, where available, were tracked down for each sequence (Table S1).

In order to assess the performance of the ITS in terms of resolution and clade fidelity, we analyzed six additional markers for comparison for which sufficient data were available, namely nuLSU, IGS, $\beta$-tubulin (TUB2), $R P B 1, R P B 2$, and $M C M 7$, including unpublished $R P B 1$ and $M C M 7$ sequences from the thesis work of Araujo (2016) and from the study by Gerlach et al. (2019a). The data sets were extracted from the originally downloaded data set and treated in the same way as the ITS (see above), resulting in six additional alignments (Files S2-S7), with the following sizes and lengths: nuLSU = 154 terminals, 872 bases; IGS $=488$ terminals, 410 bases; $T U B 2=204$ terminals, 342 bases; $R P B 1=723$ terminals, 710 bases; $R P B 2=154$ terminals, 776 bases; $M C M 7=$ 398 terminals, 541 bases. These markers were analysed without outgroup and rooted with the Dolichousnea-Eumitria clade (e.g., Divakar et al. 2017).

Overall, ITS was best represented among the seven markers with more than two times as many terminals as the second most frequently sequenced marker $(R P B 1)$. The length of the ITS alignment was in the median range, surpassed by nuLSU, $R P B 1$, and $R P B 2$, but substantially longer than $M C M 7$, IGS, and TUB2. The total size of the 
characters $\times$ terminals matrix over all markers was nearly 2.4 million data points.

Phylogenetic analysis. For the ITS and each of the other six markers, a best-scoring maximum likelihood tree was reconstructed in RAxML 8 (Stamatakis 2014), in case of the ITS and RPB1 with RAxML-HPC Blackbox 8.2.12 in the CIPRES Science Gateway (Miller et al. 2010) and for the other markers locally with RAxML 8.2.0. For all markers, we used the universal GTR-Gamma model with either 1,000 bootstrap replicates (locally) or with an automatically determined number of non-parametric bootstrap replicates (456 in case of the ITS) using a saturation criterion (CIPRES).

In the assessment of the resulting phylogenies, we used the term clade for monophyletic and the term grade for paraphyletic groupings.

Pairwise identity threshold analysis. For selected species complexes, we performed an assessment of phylogenetic resolution in the ITS by computing the pairwise BLAST identity matrix in BioEdit 7.2.5 (Hall 1999, 2011). The symmetrical matrices were then shaded using $98.5 \%$ as threshold, following the common use of this value in reference databases (Irinyi et al. 2015; Jeewon \& Hyde 2016; Kõljalg et al. 2019; Nilsson et al. 2019), as well as evidence from empirical studies (Lücking et al. 2014; Garnica et al. 2016; Vu et al. 2016, 2019; Moncada et al. 2020a).

While there are no fixed similarity thresholds between species, the $98.5 \%$ level is useful to assess potential structure in the sequence data of a complex clade. Thus, phylogenetically defined lineages within species complexes, including nested clades opposed to paraphyletic residuals (see above), were considered to potentially represent distinct species when pairwise identity values between members of different lineages were consistently $98.5 \%$ or lower.

\section{Metadata assessment}

Using the ITS voucher table (Table S1), we assessed various categories of metadata, including geographic origin (country or region), original paper where sequences were first published, year of publication, alternative identifications when differing between GenBank submission and original paper, and either the revised identification after studying the voucher material or the likely identification based on phylogenetic context and published data.

In order to assess the taxonomic and geographic representativity of the known ITS sequences, we assembled a global checklist for species of Usnea s.lat., mostly based on recent publications (after 1970), including monographic revisions and biotic surveys and in plausible cases also non-taxonomic studies, such as biochemical analyses of selected Usnea species. All names were corrected for established synonymies following most recent studies. The final list had 5,066 entries (Table S2), corresponding to a total of 735 names including currently accepted synonyms, based on 260 references.

\section{Results and discussion}

\section{Data quality}

Sequence quality. Among the 1,757 ITS sequences initially compiled, four had to be removed as they did not represent species of that genus, but other Parmeliaceae. All four are from an unpublished study from South Korea submitted by S.-H. Jang et al. in 2018 (MH258910, MH258911, MH258912, MH258913). All other sequences were confirmed as belonging to Usnea s.lat., resulting in an accuracy of $99.8 \%$ in terms of correct labeling at genus level (Table 1). Two further, also apparently unpublished sequences deposited by C. R. A. Stewart et al. in 2018 (MH887539, MH887540) had to be removed, as they originated from combined nuSSU-ITS-nuLSU tandem repeats, but did not contain the variable ITS1 and ITS2 portions. This left a total of 1,751 ingroup sequences.

Twelve sequences were determined as limited to low quality, due to a higher number of ambiguous base calls $(>3)$, often associated with additional odd base calls (File S1; Table S1), including Usnea sp. (KM369422: 15 ambiguous sites), U. florida (AF117996: 14), U. intermedia (A. Massal.) Jatta (JN009731: 8), U. cladocarpa Fée (KY021905: 4), U. silesiaca Motyka (KU352630: 4), and U. subparvula A. Gerlach \& P. Clerc (KY021928: 4). Particularly notable were four sequences of $U$. articulata (L.) Hoffm. (JN086277: 6; JN086278: 19; JN086279: 13; JN086280: 14) and two labeled $U$. dasaea Stirt. (JN086283: 16; JN086284: 15). In general, low quality did not affect the correct placement of the affected sequences, but often considerably reduced support for the containing clades (Fig. S1). In the case of the two sequences labeled $U$. dasaea from Portugal, the ambiguous base calls occurred principally in the second portion of the ITS2 and were associated with numerous odd base calls, resulting in an extremely long stem branch (see below). These were the only two instances where the underlying topology was considered unreliable due to potential issues with sequence quality.

Table 1. Quality assessment for ITS sequences and their metadata in Usnea s.lat. We used the following grading system: excellent $(\geq 90 \%$ and $\leq 100 \%$ ); very good $(\geq 80 \%$ and $<90 \%)$; good $(\geq 70 \%$ and $<80 \%$ ); satisfactory $(\geq 50 \%$ and $<70 \%)$; moderate $(\geq 30 \%$ and $<50 \%)$; poor $(\geq 10 \%$ and $<30 \%$ ); bad $(\geq 0 \%$ and $<10 \%)$.

\begin{tabular}{lcl}
\hline Category & Value & Assessment \\
\hline Genus identification & $99.8 \%$ & excellent \\
Sequence quality & $95.9 \%$ & excellent \\
Voucher information & $76.0 \%$ & good \\
Precision of identifications & $77.5 \%$ & good \\
Taxonomic diversity & $26.4 \%$ & poor \\
Weighted taxonomic diversity & $55.1 \%$ & satisfactory \\
Geographic coverage & $41.7 \%$ & moderate \\
Taxon-region records & $18.4 \%$ & poor \\
Group-region representativity & $34.4 \%$ & moderate \\
Topical diversity & - & excellent \\
Identification success & $70.6 \%$ & good \\
\hline
\end{tabular}


Occasionally, odd base calls in otherwise conserved regions within a clade were observed in 34 sequences, including two of low quality (File S1; Table S1). These also did not seem to affect phylogenetic placement as indicated by the underlying identifications and other metadata, but in some instances clade support. A total of 103 sequences exhibited conspicuous short gaps particularly in the second portion of the ITS1, including two of low quality (File S1; Table S1). The gaps appeared in unrelated clades in the same positions and were not lineage-specific. Their causes are unknown, but likely represent artifacts of the corresponding sequencing approach. Except for a lack of potentially synapomorphic signal in the gappy regions, the phylogenetic position of the sequences was not affected by these gaps. The same applied to ten short sequences missing larger portions at the $3^{\prime}$ or 5 ' end (File S1; Table S1).

Using a scoring scheme of $3=$ high quality $(1,598$ sequences), 2 = gappy or short (103), 1 = odd base calls (34), and $0=$ low quality (12), with a given sequence receiving the lowest possible score when two or more limiting criteria applied, the 1,751 sequences thus received an overall quality score of 5,054 out of a maximum of 5,271 , or $95.9 \%$. Therefore, sequence quality of available ITS data in the genus Usnea was considered very high overall (Table 1).

Wirtz et al. (2008) gave eight ITS sequences in subgenus Neuropogon as potential recombinants (Table S1). This suggests they were composed of portions representing at least two distinct lineages in a chimeric combination, e.g. in the ITS1 vs. ITS2. However, we could not detect issues with these eight sequences that would indicate them as potentially chimeric. Chimeric sequences composed of portions of two different taxa would be expected to fall on long, yet unsupported branches, which was not the case for any of these eight sequences (Table S1; Fig. S1). Rather, they appeared to represent variants comparable to those in other instances.

Voucher information. Several studies had issues with incomplete or erroneous voucher information or lacked voucher information altogether. In Thell et al. (2002: 341-342, Table 2), two specimens had the same accession number (AF451739) for the ITS, namely Flavocetraria cucullata (Bellardi) Kärnefelt \& A. Thell (DNA-932) and Usnea florida (DNA-840). The latter corresponded to the deposition in GenBank, whereas the erroneous accession number for the Flavocetraria was a lapsus for AF451793. Twelve accessions of Neuropogon (EF179795-EF179806) were indicated in GenBank to correspond to the study by Wirtz et al. (2006), but were not used or cited in that work; therefore, their geographic origin could mostly not be ascertained.

Wirtz et al. (2008) generated 71 new ITS sequences for subgenus Neuropogon, but instead of a detailed voucher table, the accession numbers were cited as a single text string: 'All sequences, including the haplotype information, were submitted to GenBank [accession nos: EF492146-EF492216 (ITS) ...' (Wirtz et al. 2008: 477). In the morphological species assignment (Wirtz et al. 2008:
474 , table 1), with detailed voucher information, the haplotypes were indicated, but not the isolate numbers, so in some cases, the GenBank accessions could not be unambiguously linked to voucher information. For instance, haplotype 17 corresponded to five accessions (EF492152, EF492158, EF492181, EF492198, EF492202), whereas in the table this haplotype was indicated for six samples from four localities, one from Ecuador, three from Argentina, and two from two different places in Antarctica. The same applied to haplotype 8. For six accessions (EF492146, EF492147, EF492153, EF492161, EF492213, EF492216), no haplotypes were indicated. These were given in the tree figure (Wirtz et al. 2008: 478, fig. 1) as 'potential recombinants' (see above). Their geographic origin could be inferred by comparing the isolate numbers with those of haplotypes indicated in the table. Two further 'potential recombinants' given in the tree figure (Wirtz et al. 2008: 478, fig. 1), with the isolate numbers 208-19 and 221-1, were apparently not included in the GenBank submissions. Upon request, we received the complete voucher table from the original author (N. Wirtz, pers. comm. July 2020), which enabled us to fill in most of the missing data, except geographic origin in 16 cases (Table S1).

Ohmura (2008) generated 24 new ITS sequences from 24 specimens for a study of Usnea rubicunda Stirt. and $U$. rubrotincta Stirt. However, only six unique sequences were submitted to GenBank, presumably subsuming specimens with sequences representing identical haplotypes under a single accession. However, each GenBank accession only included a single voucher and not all specimens corresponding to that haplotype. A similar approach was taken by Ohmura \& Clerc (2019), with three accessions (LC479120, LC479122, LC479123) representing a total of 15 specimens of $U$. cornuta, but in GenBank only citing one specimen each. For taxonomic studies, this practice is not recommended. Ideally, specimen-based Sanger sequences should relate to voucher specimens in a 1:1 ratio. If multiple specimens share the same haplotype and are represented in a single accession, all specimens should be cited with that accession in GenBank. The current submission tools do not actually allow this for structured data, but the additional specimens could be listed in the title of the representative sequence.

The voucher table submitted as a supplementary file by Millanes et al. (2014) contained detailed voucher information, but in the final version the authors missed to replace the ' $\mathrm{X}$ ' with the actual accession numbers of the newly generated sequences, including 26 of the ITS. In that case, it was possible to associate the accession numbers with the vouchers using the isolate numbers. In their broad study on mycobiont-photobiont associations of New Zealand Usnea, Buckley et al. (2014) did not provide a voucher table for the 265 samples. The GenBank accession numbers were subsequently added in a correction, but only indicating the ranges, without any specific locality information associated with each sequence.

A series of 52 accessions (JN943506 through JN943562 p.p.) was indicated in GenBank as corresponding to the barcoding studies by Kelly et al. (2011) and Schoch et al. (2012). The accessions were not cited in either work, but 
the biomaterial numbers indicate that these were duplicate submissions of sequences already submitted in Kelly et al. (2011) under another number series (e.g., JN943506 $=$ FR799053, JN943562 = FR799085, etc. $)$. Four additional accessions (JN943507, JN943508, JN943512, JN943514) were published twice as newly generated sequences. In the Swiss barcoding study by Mark et al. (2016b), seven accessions, corresponding to the genus Usnea (KX132919, KX132920, KX132921, KX132928, KX132929, KX132930, KX132932), were published with incorrect voucher information as the entries were apparently shifted in the voucher table (Mark et al. 2016b: 689, table 1).

Excluding the 147 ITS sequences provided by the second and third author of this paper (MRAN, EAC), which were submitted to GenBank as part of this study, as well as 143 unpublished sequences, 438 out of the remaining 1,461 accessions $(30.0 \%)$ had issues with proper voucher information. For 99 of these, the information could be inferred by cross-comparison with metadata, whereas for $339(77.4 \%$ of the problematic cases and $23.2 \%$ of all accessions), important voucher information such as locality data could not be resolved from published information alone.

Using a scoring scheme of $3=$ voucher information complete in original publication ( 1,023 sequences), $2=$ incomplete or erroneous but could be reconstructed from GenBank metadata or other sources (99), 1 = incomplete or erroneous and could not be reconstructed (62), and $0=$ absent (277), the 1,461 sequences originally published in corresponding scientific papers thus received a total score of 3,329 out of a maximum of 4,383 , or $76.0 \%$. Therefore, the quality of published voucher information for these sequences could overall be considered good when using a standard grading scheme (Table 1). While 'good' might seem a desirable result for basic voucher information, such as sample ID and geographic origin, one would, however, expect no less than $100 \%$, so in relative terms $76 \%$ is a poor result.

A very positive aspect in some studies was that the secondary chemistry of the underlying vouchers was listed, either in the voucher table (Truong et al. 2013a; Gerlach et al. 2017) or in other sources of information (e.g., Nadel 2016; Mark et al. 2016a: 510-511, fig. 1; Gerlach et al. 2019a: 131, table 1; Temu et al. 2019: 256, fig. 3). Given that current taxonomy in Usnea for many taxa assumes chemical variability and so the applied name is not necessarily a reflection of the underlying chemistry, the inclusion of the chemotype in the voucher information is strongly recommended for phylogenetic and DNA barcoding studies.

Identifications. Of the 1,604 Usnea sequences deposited in GenBank (excluding those provided by EAC and MRAN submitted as part of this study), 1,168 were identified to species, 64 provisionally to species using the prefix 'aff.' (60) or 'cf.' (4), 19 provisionally to species using the designation 'sp.' plus a number, and 353 were only identified to genus. The 19 provisionally identified species using 'sp.' plus a number were from four taxonomic studies (Truong et al. 2013a, 2016; Gerlach et al. 2017, $2019 \mathrm{a}$ ), in anticipation of further work to resolve the taxonomic status of this material (e.g., Gerlach et al. 2020). Of the 353 unidentified sequences, 33 were also from taxonomic studies (Thell et al. 2004; Wirtz et al. 2006, 2008, 2012; Shen et al. 2012; Gerlach et al. 2019a). Three further sequences were from a study on lichenicolous Biatoropsis species on Usnea, with the host lichens left unidentified (Millanes et al. 2014), and another seven from environmental or general barcoding studies (Kelly et al. 2011; Jaouen et al. 2019; Canini et al. 2020). A total of 32 unidentified sequences deposited in GenBank have apparently not been published, their dates of deposition ranging between 2010 and 2020 (Table S1). We assume that the more recently deposited sequences are currently in the process of being published in peer-reviewed studies. The bulk of the unidentified depositions originated from studies on mycobiont-photobiont associations of New Zealand Usnea (Rafat 2014; Buckley et al. 2014; Rafat et al. 2015).

For 112 accessions, the identification given with the accession in GenBank did not match the identification in the corresponding original publication (Table S1). In 24 cases of accessions labeled Usnea sp., precise identifications were provided in the actual papers (Shen et al. 2012; Buckley et al. 2014; Rafat et al. 2015), but not updated in the GenBank accessions. In six instances, the label Usnea sp. was either specified as Usnea sp. 1 or as Usnea lineage 14A in the corresponding publication, i.e., denoting specific taxa without a formal name awaiting further work (Wirtz et al. 2006; Gerlach et al. 2019a, 2020). In three cases (MF669881, MF669883, MN080241), the full identification provided in the publication deviated from that of the GenBank accession (Gerlach et al. 2019a; Temu et al. 2019). For 65 accessions, the labels given in the principal components of the published work (voucher specimens, phylogenetic trees) differed from those submitted with the accessions. In this case, the labels on the accessions were the correct ones, but the corresponding identifications were published in the paper in somewhat cryptic form at the end of the discussion (Wirtz et al. 2008: 482): "The following species could be identified within a collection of specimens, which were a priori assigned to two species, U. perpusilla and U. sphacelata: (1) Usnea sp. 1, (2) Usnea sp. 3, (3) Usnea ushuaiensis, (4) U. perpusilla, and (5) U. lambii (Usnea sp. 2).' Most of these issues have been rectified in the updated voucher table assembled for this study (Table S1), in part with assistance by the original authors. Overall, these issues resulted in a conflict between submitted and published label information, or in reduced precision in GenBank accessions compared to published voucher identifications for 41 entries.

Excluding the 147 ITS sequences provided by EAC and MRAN that were submitted to GenBank as part of this study, we used a scoring scheme of $3=$ precise identification to species, identical in submission and publication ( 1,133 sequences); 2 = imprecise identification to species (aff., cf., sp. 1, etc.), identical in submission and publication (83); 1 = identification different in submission and 
publication (41); and $0=$ no identification to species (sp.), identical in submission and publication (306). Applying this scheme, the total for all 1,604 sequences was 3,729, out of a maximum of 4,812 , or $77.5 \%$, resulting in an assessment of 'good' for the quality of the precision of the identifications (Table 1). It should be noted that this does not extend to their implied accuracy, which was assessed in detail a phylogenetic context (see below).

Overall, the Usnea data set underlined the common problem of mismatch between identification labels on submitted accessions versus published information, both in the original papers or regarding subsequently published taxonomic or nomenclatural changes, with original submitters often not updating their records (Schoch et al. 2017), although in the present case, the proportion of such cases was low.

\section{Metadata diversity}

Taxonomic diversity and coverage. The 1,751 ITS accessions corresponded to a total of 118 different names, 105 precise names in the accession labels, four additional names as components of imprecise identifications in the accession labels, and nine further names in the corresponding publications based on subsequently published corrections compared to the original accessions (Table S1). While Thell et al. (2012) and Lücking et al. (2017a) gave the total number of accepted species for Usnea s.lat. as 350 , our global survey revealed a total of 447 currently accepted names, a figure much higher than previously estimated. Of these, three corresponded to Dolichousnea, 21 to Eumitria, 24 to subgenus Neuropogon, and 399 to Usnea s.str. excluding subgenus Neuropogon (Table S2). Thus, overall the names originally applied to the ITS sequences represented $26.4 \%$ of the currently recognized species richness in Usnea s.lat. (Table 1, Fig. 2). Dolichousnea was the only group represented by all (three) currently recognized species (Ohmura 2002; Articus 2004).

The number of species corresponding to Eumitria is difficult to assess. A total of 14 names have been

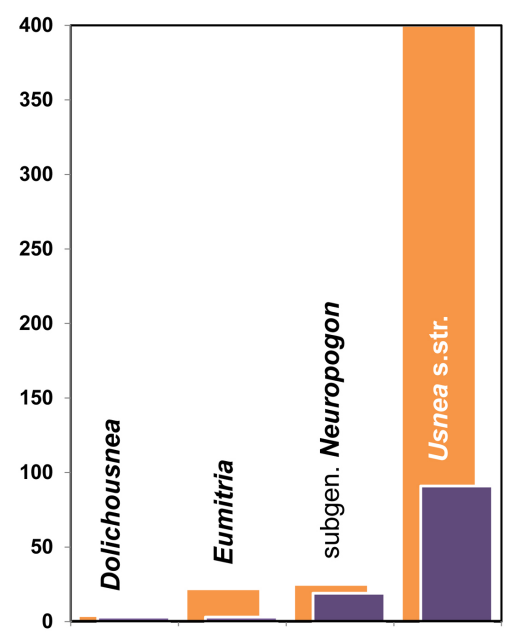

Figure 2. Number of species per genus or genus group in Usnea s.lat. (orange) and number of species sequenced by comparison (purple). formally associated with this genus. However, seven of these names (besides several others under Usnea) are considered synonyms of E. baileyi Stirt.: E. antillarum Vain., E. asperrima (Müll. Arg.) Vain., E. endochroa Vain., E. endorhodina Vain., E. formosa Stirt., E. implicita Stirt., E. perrubescens Vain., and E. tasmanica (Müll. Arg.) Vain. (Rogers \& Stevens 1988; Truong \& Clerc 2013; Buaruang et al. 2017). At least four names represent distinct species in Eumitria, namely E. firmula Stirt., E. liechtensteinii (J. Steiner) Vain., E. pectinata (Taylor) Articus, and E. trullifera (Nyl.) Vain. (Swinscow \& Krog 1974, 1986; Ohmura 2002; Articus 2004; Ohmura \& Kanda 2004; Nadel 2016; Buaruang et al. 2017; Temu et al. 2019).

More than a dozen additional names have been associated with Eumitria, but without formal combinations into that genus, mostly from Africa, but also from North America and Asia, including Usnea antigua Swinscow \& Krog, U. brunnescens C.W. Dodge, U. cervicornis C.W. Dodge, U. congdonii Krog, U. cristata Motyka, U. elata Motyka, U. flaveola Motyka, U. perplectata Motyka, U. pulvinulata C.W. Dodge, U. recurvata J.D. Chao, L.W. Hsu \& Z.M. Sun, U. subcristata C.W. Dodge, U. subflaveola Truong \& P. Clerc, U. subrectangulata J.D. Chao, L.W. Hsu \& Z.M. Sun, U. uluguruensis Krog, U. vainioi Motyka, U. welwitschiana Motyka, and U. zombensis Krog (Chao et al. 1975; Swinscow \& Krog 1986; Rogers \& Stevens 1988; Krog 1994; Truong \& Clerc 2013; Temu et al. 2019). The Eumitria pectinata complex consists of pendulous species with an almost solid, brown-pigmented axis that only partially becomes fistulate, including also $U$. mexicana Vain. and its relatives or putative synonyms, U. chloreoides Motyka, U. duriuscula Motyka, U. gigas Motyka, and U. himantodes Stirt. (Swinscow \& Krog 1988; Herrera-Campos et al. 1998; Ohmura 2001; Truong et al. 2013b; Nadel 2016; Temu et al. 2019). Our global survey indicates that Eumitria currently contains about two dozen named species, only two of which had ITS sequence accessions in GenBank, namely E. baileyi and E. pectinata (Ohmura 2002; Orock et al. 2012; Jaouen et al. 2019; Temu et al. 2019). One unidentified species from China had GenBank accessions that have not yet been published. Whether this species corresponds to a new or a known species is unclear. In addition, the unpublished thesis work on Usnea from São Tomé and Príncipe (Nadel 2016) included ITS sequences for E. firmula (Table S1). Thus, as a whole, only around $12 \%$ of the species of this genus currently have ITS sequence data (Fig. 2).

Walker (1985) accepted 15 species under the concept of subgenus Neuropogon. Of these, U. acanthella and $U$. durietzii do not belong to Neuropogon (Wirtz et al. 2006). Eight species represent the core clade of subgenus Neuropogon, including U. acromelana Stirt., U. antarctica, U. aurantiacoatra, U. patagonica F.J. Walker, U. perpusilla (I.M. Lamb) F.J. Walker, U. sphacelata R. Br., $U$. subantarctica F.J. Walker, and U. trachycarpa (Stirt.) Müll. Arg., to which the recently recognized $U$. lambii (Imshaug) Wirtz \& Lumbsch and U. ushuaiensis (I.M. Lamb) Wirtz, Printzen \& Lumbsch can be added (Articus 2004; Wirtz et al. 2006, 2008, 2012; Seymour et al. 


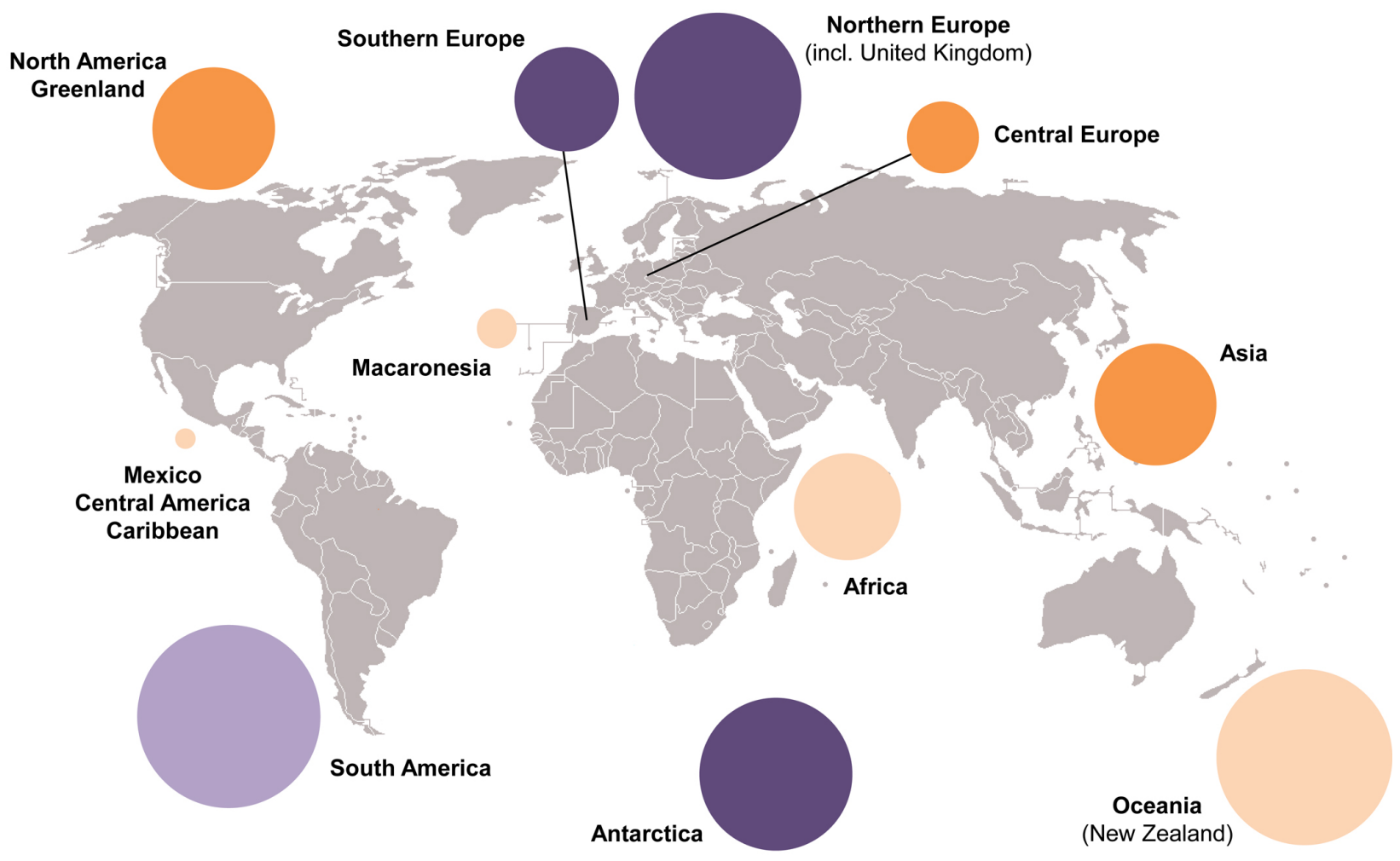

Figure 3. Geographic coverage of available ITS sequences in Usnea s.lat. The area of the circles is proportional to the number of sequences. Purple = well-sampled, lilac = moderately well-sampled; orange = moderately sampled; beige = poorly sampled (including geographically biased sampling and/or high proportion of unidentified samples). Base map taken from Wikimedia Commons [https://commons.wikimedia.org/wiki/ File:BlankMap-World-v2.png].

2007; Cao et al. 2015, 2018; Lagostina et al. 2018). Two species treated in Walker (1985) under subgenus Neuropogon clustered in a sister clade, namely Usnea ciliata (Müll. Arg.) Vain. and U. subcapillaris (D.J. Galloway) F.J. Walker (see also Wirtz et al. 2006). This clade of 148 terminals was almost exclusively formed by samples from New Zealand, with the exception of one specimen from Antarctica and another from Chile (Fig. S1). Most of the samples were not identified (Buckley et al. 2014), but the few identified specimens (Rafat et al. 2015) include two names also associated with subgenus Neuropogon by Walker (1985), namely U. inermis Motyka and U. torulosa (Müll. Arg.) Zahlbr. Three further identifications in this clade, U. ciliifera Motyka, U. pusilla (Räsänen) Räsanen and U. xanthopoga Nyl. (Rafat et al. 2015), have not been associated with Neuropogon by Walker (1985). Another three taxa accepted by Walker (1985), U. neuropogonoides Motyka, U. pseudocapillaris F.J. Walker, and U. taylorii Hook. f., have not yet been sequenced. Thus, the core group of Neuropogon was found to be rather well represented by ITS sequence data (Fig. 2), both in terms of species and in the number of terminals, with a total of 446 (27.8\% of all Usnea ITS accessions). In contrast, the New Zealand sister clade, while also containing a large number of samples ( $9.2 \%$ of all accessions), will require substantial reassessment of the missing taxonomic identifications to allow for a reliable interpretation of its taxonomic composition.

After the removal of species in Dolichousnea and Eumitria, and separating species in subgenus or sect.
Neuropogon, Usnea s.str. retained 399 taxa in our global checklist (Table S2), representing the bulk of the known species richness in Usnea s.lat. Of these, 91 names (23.5\%) were represented among ITS accessions (Fig. 2). Overall, taxonomic diversity among available ITS sequences was biased in favor of Dolichousnea (100\% of known species sequenced) and subgenus Neuropogon (82.6\%), whereas relatively few of the known species have been sequenced in Usnea s.str. (23.5\%) and Eumitria (14.2\%). Giving each group equal weight, the weighted average of the taxonomic diversity represented in sequence data amounted to $55.1 \%$ (Table 1 ).

Geographic coverage. ITS sequence data for Usnea were available from all major geographic regions, but with strong bias towards Antarctica, Europe, and South America (Fig. 3). Compared to area size, by far most sequences have been generated for Oceania; however, this figure is misleading for two reasons: (1) all available sequences were from New Zealand only, none for other parts of Oceania; and (2) only a few of these sequences were actually identified. Overall, best represented was Antarctica, both regarding the amount of sequence data and the level of identifications. Many sequence data were also available for Northern Europe and South America, whereas North America, the rest of Europe, and Asia had a limited amount of data, in the latter case biased towards Japan. Central America including Mexico and the Caribbean, as well as Africa, were strongly underrepresented (Fig. 3). 
We established the following scoring system, taking into account total number of sequences and within-region bias, as well as taxonomic resolution: $3=$ well-represented (Antarctica), 2 = moderately well-represented (all of Europe, South America), 1 = limited data (North America, Asia, Oceania), and $0=$ poorly represented or no data (Central America, Africa), we computed an overall score of 10 out of 24 maximum, or $41.7 \%$, for geographic coverage (Table 1). Considering the eight regions, we also counted the number of unique taxon-region records, both for taxonomic records (946 total; Table S2) and for ITS sequence data (174 total; Table $\mathrm{S} 1$ ), resulting in a proportion of $18.4 \%$ (Table 1).

We further subdivided the unique taxon-region records by geographic region and taxonomic group (genus or subgenus) to compute a group-dependent weighted proportion of available sequence data (Fig. 4). North America and Europe were best represented regarding Dolichousnea and subgenus Neuropogon, whereas South America was biased towards Neuropogon and Usnea, Africa towards Eumitria, and Asia towards Dolichousnea and Eumitria. Dolichousnea was best represented in terms of sequence data vs. recorded species richness in Asia, Europe, and North America, Eumitria best in Asia, Oceania, and Africa, subgenus Neuropogon best in Antarctica, North America, Europe, and South America, and Usnea s.str. best in Europe, followed by North and South America and Asia (Fig. 4). Overall, there was strong taxon- and regionbased bias with the average taxon-region representativity in ITS sequence data resulting in $34.4 \%$ (Table 1). These figures give guidelines for which geographic regions

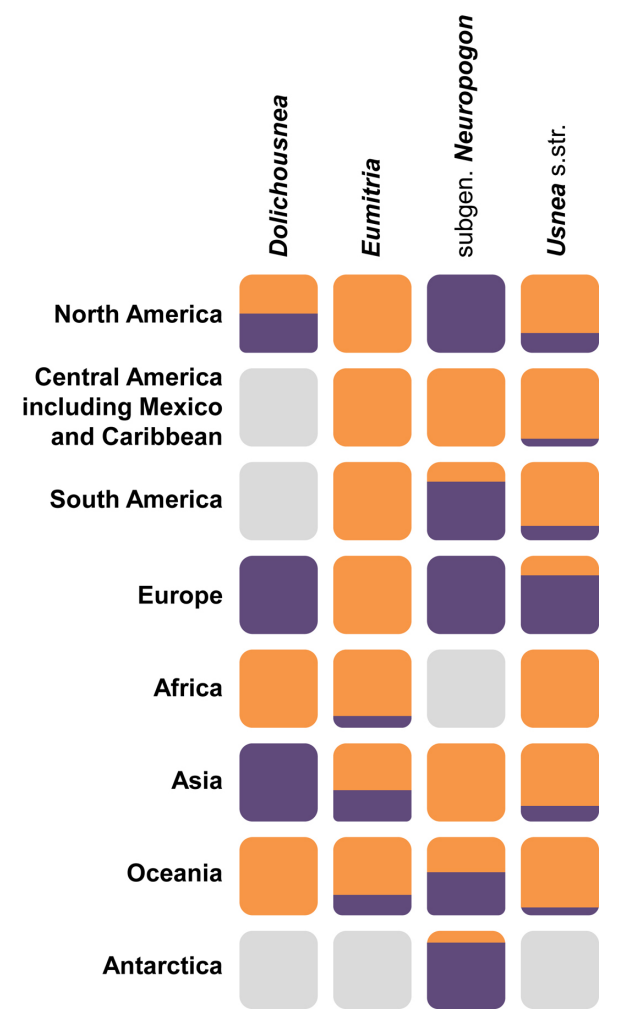

Figure 4. Group-by-region representativity of ITS sequence data for Usnea s.lat., in terms of sequenced vs. reported names per group and region. Orange $=$ reported names $($ set to $100 \%$ ), purple $=$ sequenced names (as proportion), grey = no names reported for group and region.

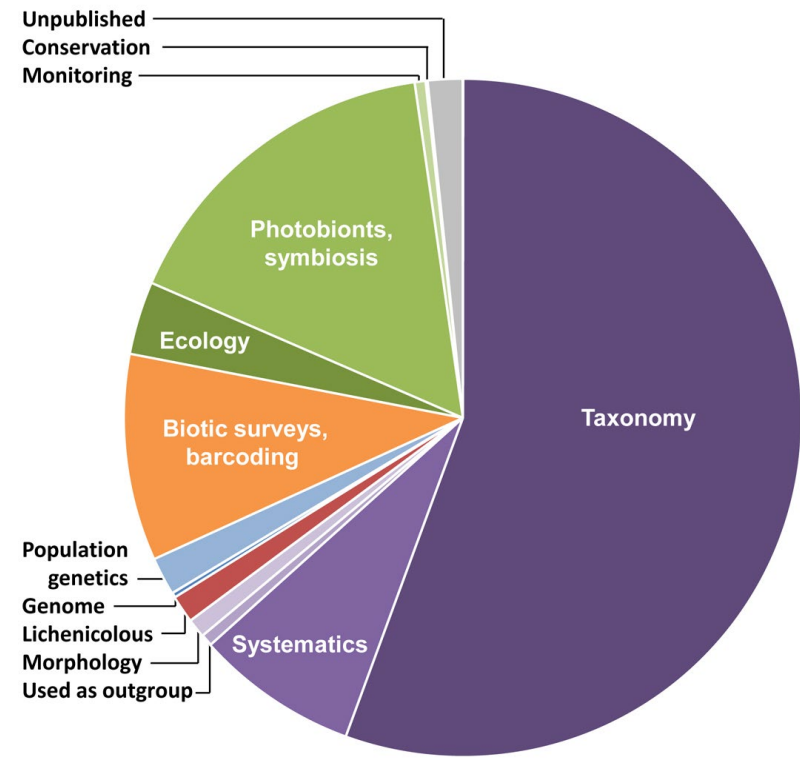

Figure 5. Topical diversity of studies producing ITS sequences in Usnea s.lat., based in the proportion of accessions pertaining to each topic.

(e.g., North and Central America, Africa, continental and tropical Asia) and taxonomic groups in those regions (e.g., Eumitria) should be preferentially targeted for further DNA-based inventories.

Topical diversity. The 1,751 ITS accessions for the genus Usnea were generated in 80 studies, 52 published, two corresponding to thesis works, and 26 representing (yet) unpublished submissions (Fig. 5). More than half (56\%) of the accessions stem from taxonomic studies focusing on Usnea s.lat. (Articus et al. 2002; Seymour et al. 2007; Ohmura 2008; Wirtz et al. 2008, 2012; Lumbsch \& Wirtz 2011; Saag et al. 2011; Shen et al. 2012; Truong et al. 2013a; Araujo 2016; Nadel 2016; Truong \& Clerc 2016; Mark et al. 2016a; Gerlach et al. 2017, 2019a, b; Lagostina et al. 2018; Ohmura \& Clerc 2019; Temu et al. 2019). A few additional papers focused on generic delimitation either within Usnea s.lat. or within Parmeliaceae (Wedin et al. 1999; Ohmura 2002; Articus 2004; Ohmura \& Kanda 2004; Wirtz et al. 2006; Arup et al. 2007). Usnea sequences were also generated as outgroups for other taxa (LaGreca 1999; Thell et al. 2002; Schmull et al. 2011; Myllys et al. 2014).

Another large block (16\%) of ITS sequences for Usnea were generated for studies on mycobiont-photobiont associations (Buckley et al. 2014; Park et al. 2014, 2015; Rafat et al. 2015), including one accession for a taxonomically diverse culturing survey (McDonald et al. 2013). The mycobiont-photobiont studies focused exclusively on New Zealand (Buckley et al. 2014; Rafat et al. 2015) and Antarctica (Park et al. 2014, 2015). The third major group of accessions (11\%) originated from floristic and (meta-)barcoding surveys (Hur et al. 2005; Kim et al. 2006; Kelly et al. 2011; Orock et al. 2012; Schoch et al. 2012; Šoun et al. 2015; Mark et al. 2016b; Jaouen et al. 2019; Lendemer et al. 2019; Marthinsen et al. 2019).

Other diverse topics included lichenicolous fungi (Biatoropsis) on Usnea hosts (Millanes et al. 2014), 
morphological variation and population genetics (Rolstad et al. 2013; Cao et al. 2018), genome sequencing (as yet unpublished), ecology (Cao et al. 2015; Greenwood et al. 2016; Canini et al. 2020), and monitoring and conservation (Ohmura 2011; Dorey et al. 2019). Each of these accounted for $1-3 \%$ of the accessions (Fig. 5). Considering that voucher-based fungal ITS sequences are almost exclusively generated for taxonomic purposes, the diversity of topics leading to ITS accessions in Usnea s.lat., and consequently the broad application of ITS barcoding also for ecological and other studies, was here considered excellent (Table 1).

Years published. As mentioned above, the first ITS sequence for Usnea s.lat. was published in 1999, with a marked increase in the generation of sequence data from 2002 onwards. However, by far, most of the available sequence data were generated in the past decade, particularly in the first half (Fig. 6).

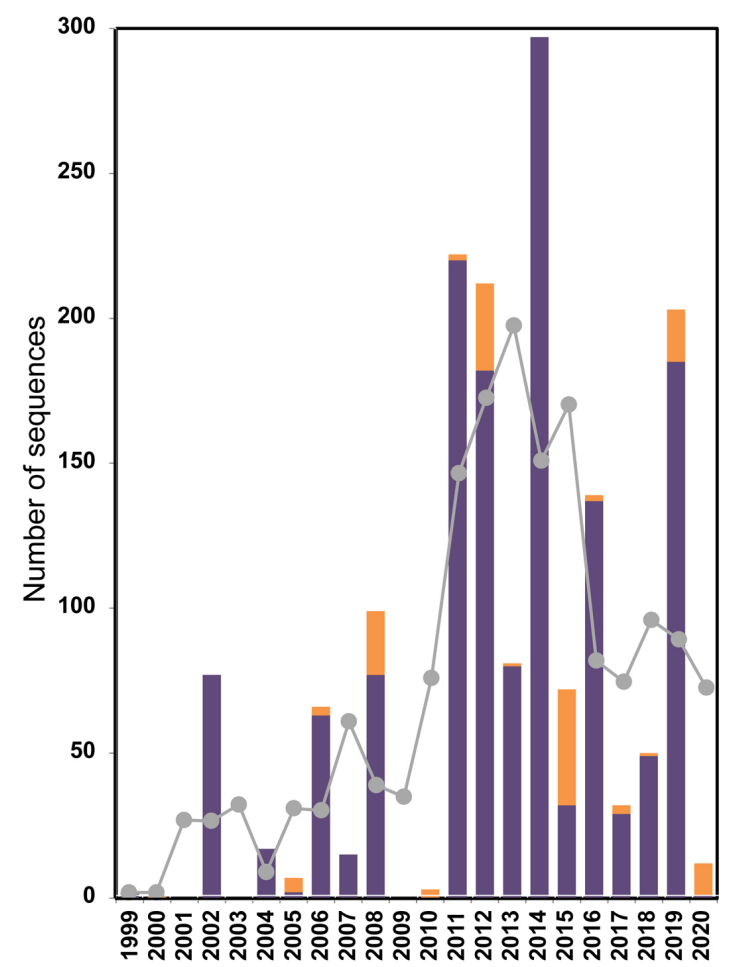

Figure 6. Temporal pattern of the generation of ITS sequence data in Usnea s.lat. Purple = published sequences (with date corresponding to publication date), orange $=$ unpublished sequences (with date corresponding to submission date). Grey dots and line denote 3-year moving average.

\section{ITS-based phylogeny}

Overall topology and genus concept. The ITS-based maximum likelihood tree of 1,751 ingroup sequences mirrored the topology obtained in other recent studies (Nadel 2016; Divakar et al. 2017; Kraichak et al. 2017), with Dolichousnea and Eumitria forming a monophyletic clade sister to Usnea including Neuropogon, and Neuropogon s.str. forming a monophyletic clade nested within Usnea s.str. (Fig. 7; Fig. S1). The sister group relationship of Dolichousnea and Eumitria was not supported, but each of the two genera was strongly supported on long stem branches (94-96\%), matching other findings that recovered both genera as supported clades, but with varying positions as early diverging lineages within Usnea s.lat. (Ohmura 2002; Articus 2004; Ohmura \& Kanda 2004; Wirtz et al. 2006). As other markers agree in these patterns, in particular the long stem branches leading to both groups (see below), we therefore follow Articus (2004) and Divakar et al. (2017) to recognize Dolichousnea and Eumitria as separate genera. Dolichousnea is thereby characterized by annular pseudocyphellae and an amyloid central axis, whereas Eumitria generally features a hollow, often fistulate central axis (Swinscow \& Krog 1974; Ohmura 2001, 2002; Articus 2004; Temu et al. 2019). Given that these features are not always easily discernable and are not fully consistent with the recognized genera (e.g., Eumitria pectinata complex, Usnea durietzii, see below), one could also defend the recognition of a single genus Usnea, maintaining the subdivision into three subgenera (e.g., Ohmura 2002; Ohmura \& Kanda 2004; Wirtz et al. 2006; Truong \& Clerc 2013; Truong et al. 2013b; Ohmura \& Kashiwadani 2018; Temu et al. 2019). However, we consider a three-genus solution a good compromise between limited phenotypic distinctiveness and the long history of independent evolution undergone by these three clades (Divakar et al. 2017).

The Usnea-Neuropogon clade was supported in our ITS-based tree, albeit at low level (71\%). The species originally included in subgenus Neuropogon by Walker (1985) were found in three positions. U. acanthella and $U$. durietzii formed two separate clades within Usnea s.str., whereas all other species clustered in an unsupported, terminal clade. Articus (2004) had recovered Neuropogon as one of four supported clades within Usnea s.lat. based on ITS data, but without backbone resolution, proposing to separate Neuropogon at genus level, although it was not reciprocally monophyletic in relation to Usnea s.str. Ohmura \& Kanda (2004), also using ITS data, obtained a more resolved backbone, recognizing three subgenera: Dolichousnea, Eumitria, and Usnea, with Neuropogon nested within the latter, and therefore proposed to treat Neuropogon as a section within subgenus Usnea. Wirtz et al. (2006), again based on ITS, found Neuropogon to be polyphyletic, the two species $U$. acanthella and $U$. durietzii, which had not been sampled in earlier studies, falling outside the monophyletic core clade. Their topology was congruent with ours. Overall, this would support the formal inclusion of neuropogonoid species within Usnea s.str., although Divakar et al. (2017), based on a six-marker data set (ITS, nuLSU, mtSSU, RPB1, MCM7, TSR 1), found the Neuropogon core clade to form an early diverging lineage with supported reciprocal monophyly in relation to Usnea s.str. These authors did not recognize Neuropogon as a separate genus, because the time of divergence was too recent under their temporal banding scheme (see also Kraichak et al. 2017, 2018). While this may not be considered a sole reason to define taxa (Lücking 2019), here we agree to maintain Neuropogon within Usnea, as the sampling in the study by Divakar et al. (2017) was limited and it cannot be assumed that 
reciprocal monophyly of the Neuropogon would be maintained in an expanded taxon set using multiple markers. To simplify our taxonomic approach, we consider $\mathrm{Neu}$ ropogon a subgenus within Usnea s.str., although the precise rank to be applied to this taxon, whether subgenus or section, remains open to interpretation, and henceforth, we use the name Neuropogon when referring to subgenus Neuropogon. Species of this group mostly have a patchy black pigmentation in the cortex and a mostly brown to black apothecial disc (Ohmura 2001, 2002; Articus 2004; Ohmura \& Kanda 2004; Wirtz et al. 2006, 2008, 2012; Lumbsch \& Wirtz 2011).

In our global ITS phylogeny, the Neuropogon clade was divided into two unsupported subclades: one was almost exclusively from New Zealand (147 out of 149 samples), plus one specimen from Antarctica and another from Chile, both forming early diverging lineages in that subclade, whereas the other, considered the Neuropogon core clade, with 446 terminals, included samples from Antarctica, South America, North America (U. lambii, U. sphacelata), and Europe (U. sphacelata), but not a single one from New Zealand. This topology was also recovered in other studies (Wirtz et al. 2006), but the impressive, albeit unsupported division of a New Zealand vs. a non-New Zealand subclade had apparently not been noticed before. Rafat et al. (2015) obtained a different topology, with the Neuropogon core clade nested within the New Zealand clade, but this topology was not supported and likely biased by the unusual outgroup selection (Ramalina; Ramalinaceae) that may have resulted in alignment artifacts. The division of Neuropogon into two large, geographically separated clades was also remarkable because one species in the Neuropogon core clade, $U$. acromelana, was originally described from New Zealand, and two further species, U. antarctica and U. sphacelata, were reported from there (Walker 1985; Galloway 2007). Yet, although both New Zealand and Antarctica have been extensively sampled for molecular data (Wirtz et al. 2006, 2008, 2012; Seymour et al. 2007; Buckley et al. 2014; Park et al. 2015; Rafat et al. 2015; Cao et al. $2015,2018)$, these three species have not been demonstrated to be present in New Zealand based on molecular data, but have been frequently identified in sequenced samples from Antarctica and/or southern South America (Table S1). If this pattern is confirmed, it provides a taxonomic challenge, as the numerous samples from Argentina identified as U. acromelana (Wirtz et al. 2006, 2012; Seymour et al. 2007) may not belong to that species (see also below). On the other hand, given the confirmed wide distribution of $U$. sphacelata with sequence data from Antarctica, throughout South and North America and northern Europe (Table S1, Fig. S1), its occurrence in New Zealand appears likely.

The backbone of the paraphyletic Usnea s.str. grade was not supported in the ITS, although some larger subclades received support. Ohmura (2002) and Ohmura \& Kanda (2004) proposed to divide Usnea s.str. into two sections, sect. Usnea and sect. Ceratinae, neither of the two supported in that work. In the limited sampling of that study, sect. Usnea included U. fulvoreagens (Räsänen)
Räsänen, U. glabrescens (Nyl. ex Vain.) Vain., U. subfloridana, and U. wasmuthii Räsänen, whereas sect. Ceratinae contained $U$. aciculifera Vain., U. ceratina Ach., U. dasaea, U. himalayana C. Bab., U. intumescens Asahina, U. merrillii Motyka, U. mutabilis Stirt., U. nipparensis Asahina, U. pangiana Stirt., U. pygmoidea (Asahina) Y. Ohmura, U. rubicunda, and U. rubrotincta. Based on a smaller and partly different sampling, Articus (2004) recovered sect. Usnea by inclusion of U. wasmuthii, but also found additional taxa clustering in that clade, namely U. barbata (L.) F.H. Wigg., U. chaetophora Stirt. (now included in U. dasopoga), U. dasopoga (Ach.) Nyl. (as U. filipendula Stirt.), U. florida, and U. rigida (Ach.) Röhl. ex Zahlbr. (nom. illeg.; =U. intermedia). Sect. Ceratinae was only represented by $U$. ceratina. A third clade was formed by $U$. articulata, $U$. fragilescens Hav. ex Lynge, and $U$. hirta (L.) Weber, species not included in Ohmura (2002). Wirtz et al. (2006) resolved sect. Usnea, with the same species as in Ohmura (2002), Ohmura \& Kanda (2004) and Articus (2004), as well-supported clade. However, sect. Ceratinae was not recovered, forming an unresolved paraphyletic residual of early diverging lineages within subgenus Usnea. In our own global ITS-based phylogeny, neither section was recovered, although one large, unsupported subclade largely corresponded to sect. Usnea. Overall, Usnea s.str. was divided into numerous, mostly unsupported subclades with smaller, supported clades within, which are outlined and discussed in detail below.

Comparison between ITS and other markers. The nuclear large subunit of the ribosomal DNA (nuLSU) was available for three of the four groups (Eumitria, subgenus Neuropogon, Usnea s.str.). As with ITS, Eumitria (data mostly from Temu et al. 2019) was supported as early diverging lineage, whereas Neuropogon (data mostly from Lee et al. 2008) was nested within Usnea s.str. (Fig. S2). The Usnea s.str. backbone (data mostly from Schoch et al. 2012 and Millanes et al. 2014) was largely unresolved with even less structure than in the ITS. A substantial number of nuLSU sequences exhibited quality issues, particularly the occurrence of random single and contiguous gaps (File S2).

The second ribosomal DNA marker, the intergenic spacer (IGS), included sequences of Dolichousnea, Neuropogon, and Usnea s.str. As with ITS, Dolichousnea (data from Rolstad et al. 2013) was supported as an early diverging clade and Neuropogon (data from Lumbsch \& Wirtz 2011 and Wirtz et al. 2012) was nested within Usnea s.str. (Fig. S3). In contrast to ITS and nuLSU, IGS offered a surprisingly high level of supported resolution in the Usnea s.str. backbone: while U. ceratina was supported as an early diverging clade, the remainder of Usnea s.str. formed a supported sister group relationship with Neuropogon, and sect. Usnea was resolved as a large, well-supported clade. Terminal resolution also surpassed that found in ITS for the same clades and taxa (see details below). While the observed backbone support for sect. Usnea may in part be due to most of the data focusing on this particular group (Mark et al. 2016a), it appears 
that IGS surpasses ITS in performance both at deep and terminal nodes in this genus.

In addition to the two rDNA markers, four protein-coding markers were analyzed. Of these, $\beta$-tubulin (TUB2) is a classic marker used in many fungal studies, but is known to cause potential problems with paralogs (Landvik et al. 2001; Zhao et al. 2014). The available data, mostly covering Usnea s.str. and few for Dolichousnea and Neuropogon, recovered Dolichousnea (data from Articus 2004) as strongly supported early diverging lineage and Neuropogon (data from Articus 2004) nested within Usnea s.str., albeit not in a terminal position, as in the three ribosomal markers. Usnea s.str. (data mostly from Saag et al. 2011 and Mark et al. 2016a) was divided into five strongly supported clades, with Neuropogon situated in between (Fig. S4). Notably, sect. Usnea was recovered, while sect. Ceratinae sensu Ohmura (2002) and Ohmura \& Kanda (2004) was not, with U. ceratina clearly separated from the remainder of this group. There was no indication of artifactual topologies due to potential paralogs in TUB2 in the backbone, but terminal positions particularly in sect. Usnea showed some aberrant placements (see below for details) and the terminal resolution was lower than in the ITS.

Data for RPB1 were available for Eumitria, Neoropogon and Usnea s.str., again resolving Eumitria (data from Temu et al. 2019) as supported, early diverging clade (Fig. S5). For Neuropogon (data from Lumbsch \& Wirtz 2011 and Wirtz et al. 2012), backbone solution was overall lower than for ITS, and reciprocal monophyly of the largely New Zealand-based clade was not recovered, as this clade was nested within the Neuropogon core clade. Terminal resolution in RPB1 was lower than in ITS. Within Neuropogon, we observed numerous aberrant terminal placements with regard to species otherwise well-delimited in the ITS and IGS (see details below). Notably, Neuropogon and Usnea s.str. were recovered in supported reciprocal monophyly, with Usnea s.str. including all major groupings (data mostly from Schoch et al. 2012, Wirtz et al. 2012, and Mark et al. 2016a). Both sect. Ceratinae and sect. Usnea were recovered in supported clades, together with some smaller groups. Only limited data for Neuropogon (data from Spatafora et al. 2006) and Usnea s.str. (data largely from Mark et al. 2016a) were available for $R P B 2$, and backbone resolution was much lower than for $R P B 1$. There was also indication of paralogs, such as the split of $U$. cavernosa Tuck. into two separate clades, compared to single, homogeneous clades in ITS and RPB1 (Fig. S6; see more details below).

Data for $M C M 7$ were generated in a number of studies (e.g., Truong et al. 2013a; Mark et al. 2016a; Gerlach et al. 2017, 2019a). Dolichousnea and Eumitria were resolved as early diverging lineages in a supported sister group relationship (Fig. S7), whereas data for Neuropogon were not available. Usnea s.str. formed a highly dissected, unsupported backbone and there was indication of paralog formation, as for instance in the split of $U$. parafloridana K. Mark, S. Will-Wolf \& T. Randlane $[=U$. praetervisa (Asahina) P. Clerc; see below] and $U$. wasmuthii into separate, unrelated clades, quite different from patterns seen with ITS and $R P B 1$ for these species (see more details below).

Overall, the ITS-based topology was largely congruent with that of IGS and RPBI in the delimitation of terminal clades, while backbone resolution and support were superior in the IGS and RPB1. With few exceptions detailed below, we did not observe aberrant patterns in the ITS topology, these being more frequent in $T U B 2, R P B 2$, and particularly $M C M 7$. We therefore consider the ITS a suitable marker for a first approach to molecular delimitation and identification of Usnea species with additional terminal (and backbone) resolution provided by IGS and $R P B 1$. Regarding generic delimitation within Usnea s.lat., all markers invariably resolved Dolichousnea and Eumitria as early diverging lineages, although only one marker besides ITS (MCM7) had data for both, lending strong support to their separation at genus level (see above). All markers except RPB1 gave Neuropogon as nested within Usnea s.str. The reciprocal monophyly of the two groups in the $R P B 1$ tree was not an artifact of taxon sampling and so is presumed to be genuine to this marker. Inclusion of $R P B 1$ was therefore likely responsible for the reciprocal monophyly between Usnea s.str. and Neuropogon in the multimarker study by Divakar et al. (2017).

Given the observed patterns, we interpret the aberrant topologies for particular taxa in TUB2, RPB2, and MCM7, as potential paralogs. An alternative explanation for topological conflict between markers would be hybridization and introgression through complex reticulate evolution in the recent past (Abbott et al. 2016; Widhelm et al. 2019). However, in such cases, we would expect the aberrant copies at least in part to correspond to potential 'parent' lineages (e.g., Boluda et al. 2019), which we did not observe here (see details below). Rather, the copies appear to explore new phylospace, which would be in accordance with gene duplication. Alternatively, one could imagine that these copies correspond to not yet sampled or extinct lineages (Bradshaw et al. 2020); however, we do not consider this likely as the best documented cases were detected in well-sampled species complexes. Gene duplication does not necessarily mean that orthologs and paralogs are actually present in the genome, because paralogs may eventually replace orthologs as functional genes, leading to complex situations of deviating single-copy orthologs and paralogs in different lineages of a species (Linder \& Rieseberg 2004).

Potential paralogs should not be confused with the phenomenon of incomplete lineage sorting. The latter refers to instances where, within a given clade, different markers diverge at different speed and even in different patterns, so there might be a lack of congruence between markers when it comes to delimit lineages within a clade. However, all markers should still delimit the same larger clade, i.e., there should be a deeper node of congruent ancestry. In contrast, non-homologous copies will cluster in separate clades, typically with counterparts representing the homologous copies of related clades after gene duplication occurred. Some examples are illustrated below. Given the potential presence of paralogs in TUB2, RPB2 and $M C M 7$, these markers are not recommended to assess 
species delimitation in Usnea s.lat. The best markers for this purpose appear to be IGS and RPBI in addition to ITS. We have not provided a multimarker phylogeny, as this was not the scope of this study and unfortunately, the different markers were largely generated in different studies for different purposes and taxa, and so there was overall little overlap between samples. However, broader sequencing of the IGS and $R P B 1$ as a routine approach for the phylogeny of Usnea s.lat. seems a promising approach, including for multispecies coalescence approaches as in Mark et al. (2016a) and Gerlach et al. (2019a).

In the following, the various clades and groups are discussed in detail in terms of species delimitation and the usefulness of ITS-based DNA barcoding, also including comparison between ITS and other markers.

\section{ITS-based species delimitation and DNA barcoding}

Eumitria. This strongly supported genus, which is formally accepted here following Divakar et al. (2017), formed four supported subclades based on the ITS (Fig. 7; Fig. S1), corresponding to one as yet unnamed species composed of unpublished sequences from China, deposited by S. Guo et al. in 2019 and L. Han et al. in 2020, as well as E. firmula, E. pectinata s.lat., and E. baileyi s.lat. (Ohmura 2002; Nadel 2016; Jaouen et al. 2019; Temu et al. 2019). The E. pectinata clade contained one sample (MN080241) deposited under the name E. baileyi, but correctly labeled E. pectinata in the corresponding publication (Temu et al. 2019). The unnamed species from China was internally homogeneous. The supported subclade corresponded to two consistent substitutions, resulting in a similarity of $99.6 \%$ with the other three terminals. Eumitria firmula, based on sequenced material from São Tomé and Príncipe (Nadel 2016) was also homogeneous, with minor internal variation (File S1).

The Eumitria baileyi clade formed two rather well-supported ( $88 \%$ and $89 \%$ ), reciprocally monophyletic subclades, indicating the presence of at least two species (Fig. 7; Fig. S1). This was further supported by the identity matrix (JQ673442 excluded due to reduced length), which resulted in values below $98.5 \%$ for all cross-comparisons between the two clades (Fig. 8). In addition, the early diverging singleton sequence (MW267138) in the first subclade was revealed as an additional candidate species based on the identity matrix, whereas a strongly supported $(97 \%)$ subclade in the second subclade, comprising five terminals, exhibited partial similarity overlap with the remainder of the second subclade (Fig. 8) and could at best be considered a candidate infraspecies.

Eumitria baileyi was originally described from Australia (Stirton 1881), but has since been reported from all tropical and subtropical regions (Fig. 9; Swinscow \& Krog 1988; Mies 1989; Elix \& McCarthy 1998, 2008; Stevens 1999; Aptroot \& Seaward 1999; Ohmura 2001, 2002; Galloway 2007; Mercado-Díaz 2009; Schumm \& Aptroot 2010; Singh \& Sinha 2010; Gumboski \& Eliasaro 2011; Orock et al. 2012; Truong \& Clerc 2013; Aptroot 2016; Herrera-Campos 2016; Nadel 2016; Sipman \& Aguirre-C. 2016; Rodríguez-Flakus et al. 2016; Galinato et al. 2017;
Ohmura \& Kashiwadani 2018; Bungartz et al. 2018; Esslinger 2019; Temu et al. 2019; Lücking et al. 2020c). In our analysis, the first subclade included specimens from Africa and Asia/Oceania, the latter clustering in a supported subclade. The second clade exclusively comprised African samples. The presence of geographic signal and supported phylogenetic structure within a single area, together with the notion that most of its geographic range has not yet been sampled for molecular data, indicates that E. baileyi represents a complex of several species. This is also underlined by the existence of unique phenotypes, such as an unusual specimen from the Colombian Amazon with a brightly orange inner axis (Fig. 1A). The latter may morphologically correspond to E. baileyi f. endocrocea Zahlbr. described from Taiwan (Zahlbruckner 1933: 60), although it is unclear whether the author referred to the medulla in the proper sense or the fistulate inner axis with 'Medulla ... crocea'. The secondary chemistry of $E$. baileyi s.lat. appears to be rather uniform, containing norstictic plus mostly salazinic and sometimes diffractatic acids, as well as eumitrins throughout its reported range (Ohmura 2001; Truong \& Clerc 2013; Nadel 2016; Temu et al. 2019). Some aberrant chemotypes have been reported, such as thamnolic acid, corresponding to the type of Usnea eizanensis Asahina (Ohmura 2001), and protocetraric acid in certain varieties (Swinscow \& Krog 1974, 1988). These latter are candidates possibly representing distinct species, although these chemotypes do not appear to have been sequenced yet.

Regarding the other markers for which data were available for Eumitria baileyi (nuLSU and RPB1, MCM7) all from Tanzania; Temu et al. 2019), the three available $R P B 1$ sequences (SGT110, SGT120, SGT156) were uniform (Fig. S5), although in the ITS tree the corresponding samples appeared in the two different subclades, indicating lack of resolution in the RPB1. With nuLSU, E. baileyi also formed two supported clades, but the clades were partially in supported conflict with those formed with ITS (Fig. S2). Thus, specimens SGT63 and SGT65 formed one of the two subclades in the nuLSU, but were found in the two separate subclades in the ITS. Likewise, the other subclade in the nuLSU contained eight samples (SGT110, SGT112, SGT118, SGT119, SGT120, SGT122, SGT156, SGT157), which were found in the ITS either in the first (SGT110, SGT112, SGT119) or the second subclade (SGT118, SGT120, SGT122, SGT156, SGT157). Few data were available for MCM7 (from Temu et al. 2019), with one supported conflict for sample SGT65 (Fig. S7).

The Eumitria pectinata clade exhibited internal structure, but no distinct division into reciprocally monophyletic clades. The backbone was structured into various singletons and several smaller, partly supported subclades, including one subclade with the three samples from Southeast Asia (Ohmura 2002), but without separation in the identity matrix at the $98.5 \%$ level (Fig. 10). However, three nested, well-supported subclades on longer branches appeared as candidate species in the identity matrix, including samples from São Tomé and Príncipe and/or Tanzania (Nadel 2016; Temu et al. 2019). The largely unresolved backbone had a variable chemistry including 


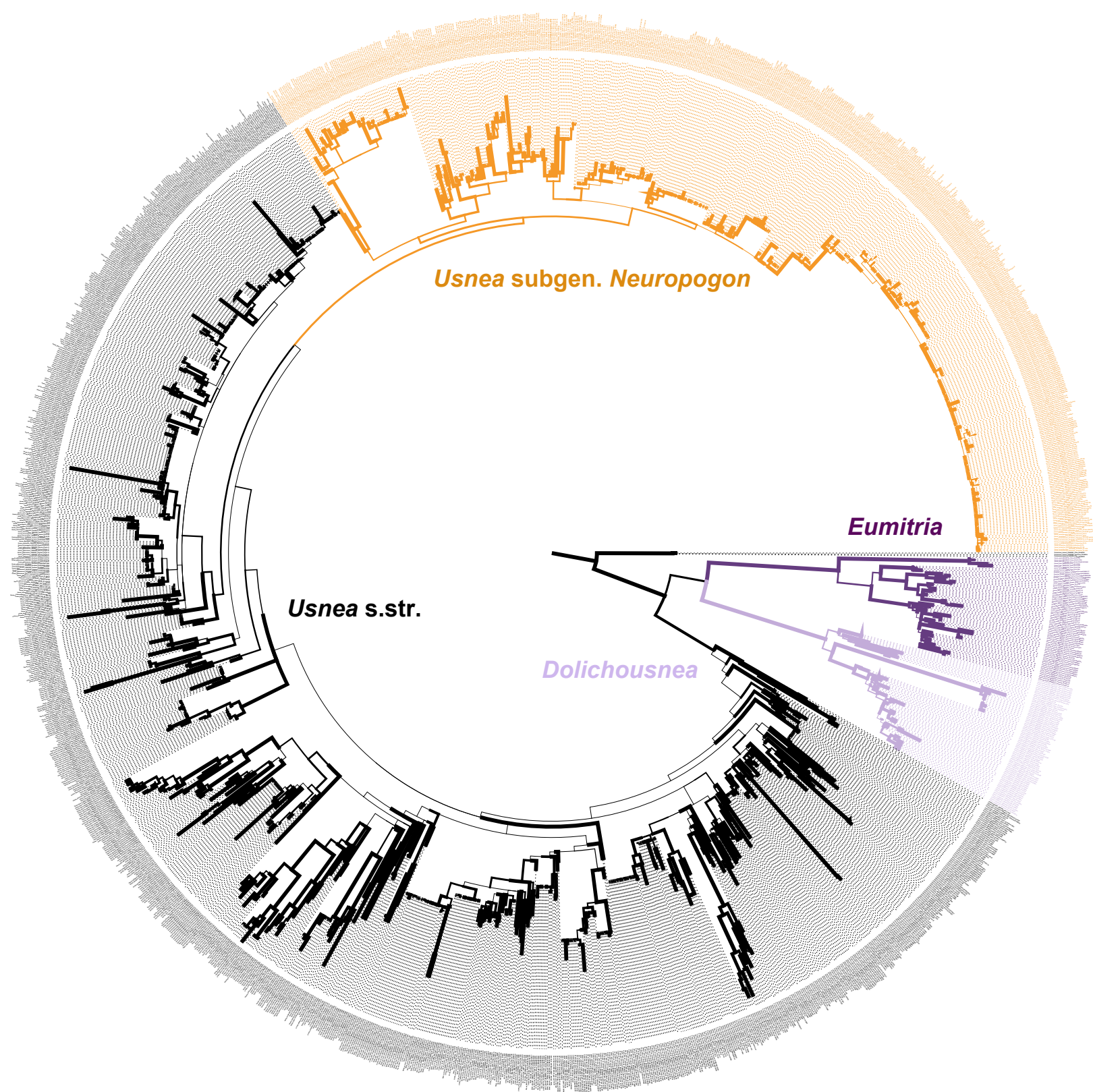

Figure 7. Global tree (best scoring maximum likelihood tree) of Usnea s.lat. based on the fungal ITS DNA barcoding marker. Figure can be enlarged to discern labels. For separate phylogram including bootstrap support values, see File S10.

constictic, salazinic, protocetraric, and/or diffractaic acids in various combinations, but never with salazinic acid and very rarely with protocetraric acid as major compounds. The three nested, supported clades on longer branches had relatively uniform chemistries deviating from the variation in the backbone: the clade composed of MN080235, MW267152, MW267153, MW267154, and MW267155, from São Tomé and Príncipe and Tanzania (relabeled E. aff. pecinata 1; Table S1) had protocetraric acid as major compound; the clade containing MW267156, MW267157, MW267158, MW267159, MN080233, and MW267160, from São Tomé and Príncipe and Tanzania (relabeled E. aff. pectinata 2) featured mostly salazinic acid as major substance (with one exception), and the clade formed by MN080238, MN080237, and MN080239, from Tanzania (relabeled $E$. aff. pectinata 3 ), had constictic and diffractaic acid (Nadel 2016; Temu et al. 2019).
Eumitria pectinata has been described from Southeast Asia, the type containing norstictic, menegazziaic, stictic, and constictic acids (Ohmura 2001). The chemistry of eastern paleotropical material is uniform and consistent with that of the type (Ohmura 2001; Nadel 2016), which means that possibly only the small clade formed by the three Southeast Asian samples represents E. pectinata s.str. and the other terminals in the backbone were therefore relabeled $E$. cf. pectinata (Table S1). The small clade relabeled $E$. aff. pectinata 3 has a chemistry matching that of Usnea mexicana, which presumably only occurs in the Neotropics (Herrera-Campos et al. 1998; Truong et al. 2013b), but has not yet been sequenced from there. In addition, at least five specimens in the backbone feature that chemistry: three from São Tomé and Príncipe (MN0063, MN0583, MN0585) and two from Tanzania (MN080240, MN080241). Although Nadel (2016) and 


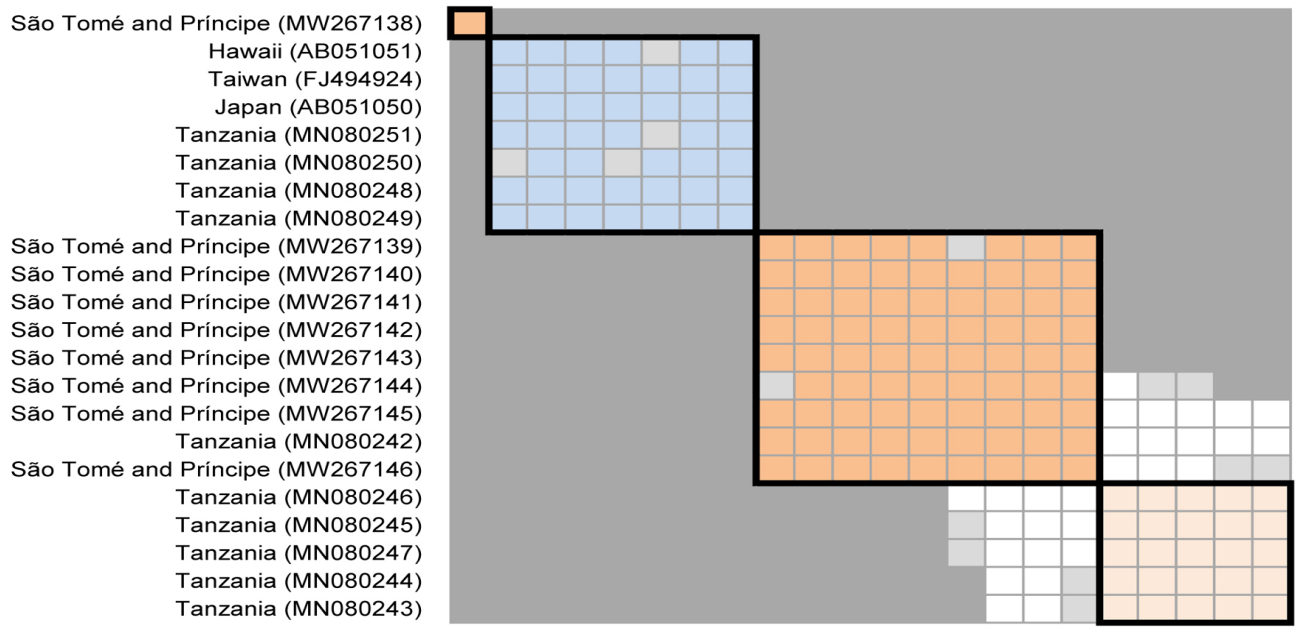

Figure 8. Pairwise identity matrix based on the ITS for the Eumitria baileyi clade. Dark grey $=$ identity below $98.5 \%$; light grey $=$ identity exactly $98.5 \%$; white, blue and beige $=$ identity above $98.5 \%$.

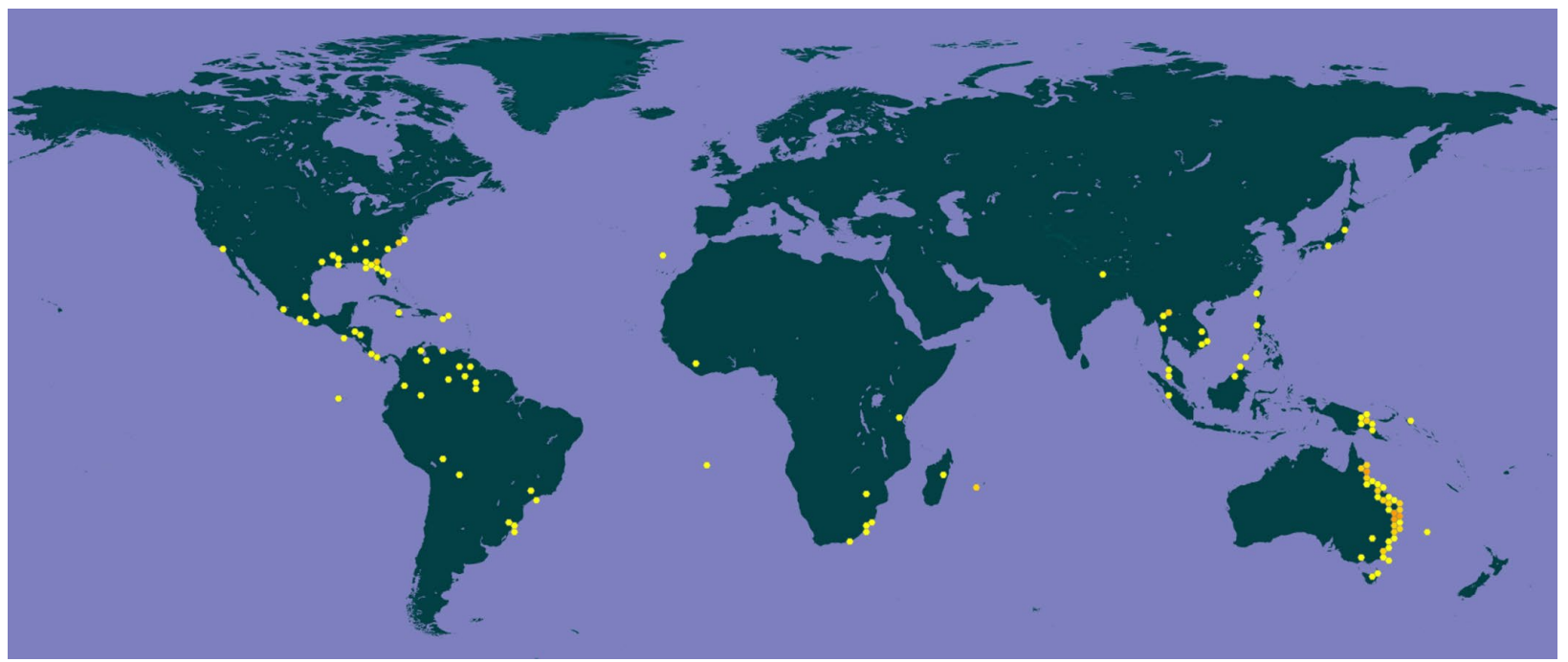

Figure 9. World distribution of Eumitria baileyi s.lat. according to the Global Biodiversity Information Facility, GBIF [https://www.gbif.org/ species/7247545].

Temu et al. (2019) do not discuss the name U. mexicana for their African material, either the small clade or part of the backbone may represent that species, implying a considerable range extension. Notably, GBIF has one record of $U$. mexicana from Cameroon, identified by P. Clerc (https://www.gbif.org/occurrence/1144796085), which would support our interpretation. A potential name for specimens containing protocetraric acid is $U$. duriuscula, described from Brazil (Truong et al. 2013b). Besides the larger subclade relabeled $E$. aff. pectinata 1 , this substance was also detected in one specimen of the other subclade (E. aff. pectinata 2) from Tanzania and a singleton in the backbone (MN080236) from Tanzania (Temu et al. 2019), so the possible application of this name remains unclear.

A sample from French Guiana (MK547011), originally identified as Usnea sp. as part of a broad fungal barcoding study (Jaouen et al. 2019), clustered with two specimens from São Tomé and Príncipe in an early diverging, supported lineage, its sequence identical to one of the two samples from São Tomé and Príncipe. Eumitria pectinata has so far only been reported from Africa and Asia
(Ohmura 2002, 2012; Singh \& Sinha 2010; Nadel 2016; Ohmura \& Kashiwadani 2018; Temu et al. 2019). Unfortunately, the chemistry of this material is not known, but given the fact that its ITS is identical to MW267148 from São Tomé and Príncipe and the latter has a mixed chemistry of protocetraric, salazinic, constictic, and diffractaic acids (Nadel 2016), whereas the specimens matching the chemistry of $U$. mexicana are separate (see above), we can exclude the possibility that the French Guianan specimen represents $U$. mexicana. It is also distinct from the clade representing E. pectinata s.str., so it is possible that this is another unrecognized candidate species.

Data available from the nuLSU (from Temu et al. 2019) were fully congruent with the ITS (Fig. S2), whereas the RPB1 (from Temu et al. 2019) provided less resolution and one supported conflict for sample SGT87, which clustered with a specimen (SGT117) representing subclade E. aff. pectinata 3 (Fig. S5).

Overall, Eumitria is in need of a much broader molecular sample of phenotypically defined species (mostly from Africa and Asia, but including species currently 


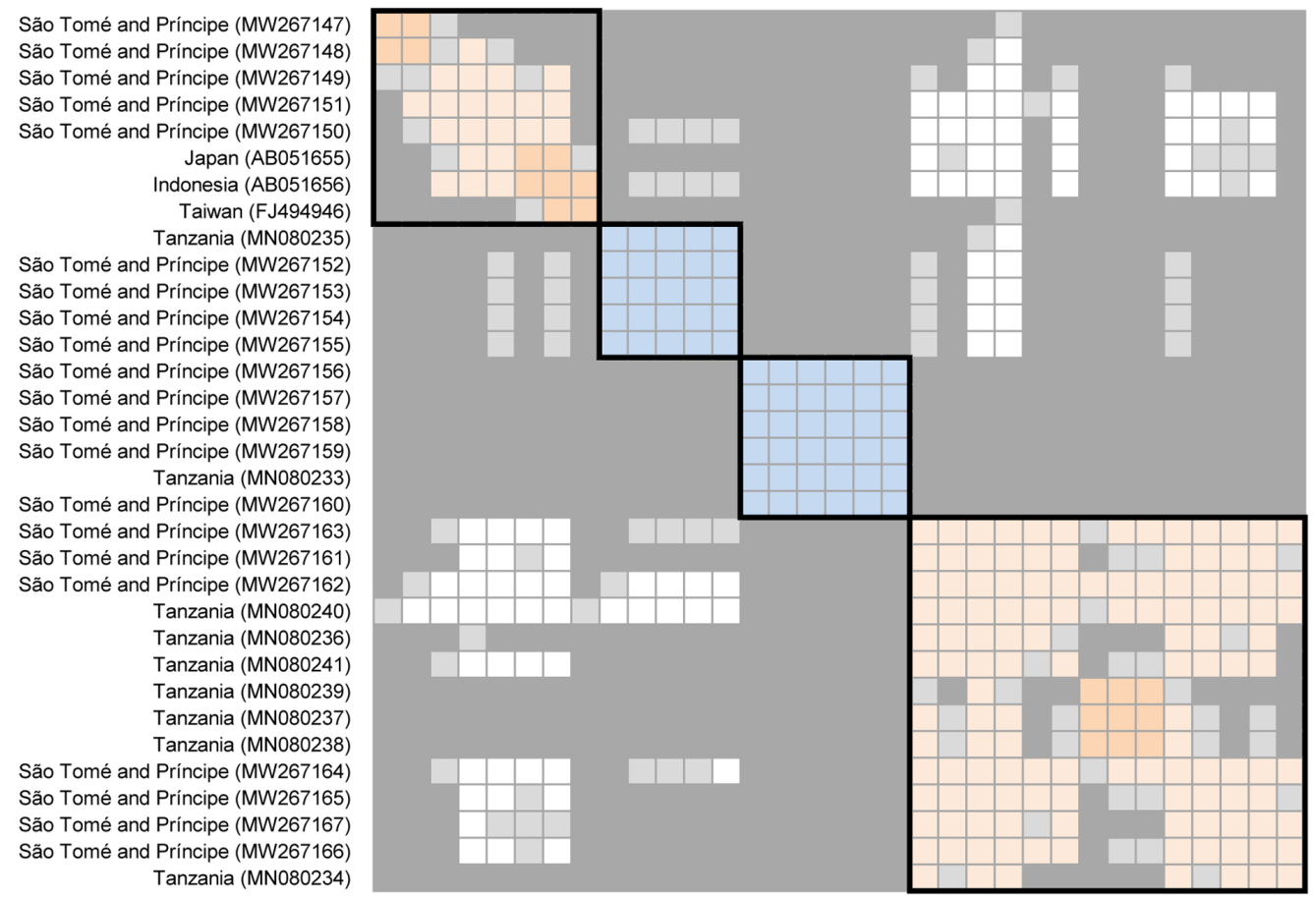

Figure 10. Pairwise identity matrix based on the ITS for the Eumitria pectinata clade. Dark grey $=$ identity below $98.5 \%$; grey $=$ identity exactly $98.5 \%$; white, blue and beige = identity above $98.5 \%$.

placed in Usnea from the Neotropics, in particular U. mexicana), as well as a detailed phylogenetic revision of species complexes such as E. baileyi s.lat. and E. pectinata s.lat., to obtain a better understanding of this genus and its species. The latter two do not seem to represent single species, but rather recently diversifying clades that can only be resolved with broad sampling using several markers. One may also argue that the observed ITS variation in these clades is overinterpreted, but given that in other instances, taxa that have identical ITS were shown to represent different species with approaches such as RADseq (Grewe et al. 2018), it seems inconsistent to assume that structured differences in the ITS, as observed in E. baileyi s.lat. and E. pectinata s.lat., may just be infraspecific variation in these cases.

Dolichousnea. Dolichousnea is by far the best sampled group in Usnea s.lat., with all three currently recognized taxa represented by ample sequence data (Figs 2, 7; Table S1; Fig. S1). The three taxa were all recovered in monophyletic clades, but exhibited different topological patterns. Dolichousnea diffracta (Vain.) Articus formed a strongly supported clade on a very long stem branch with little internal variation across its sampled range including, Japan, South Korea, and Taiwan (Articus 2004; Ohmura 2002; Park et al. 2014; and several unpublished accessions). The chemistry of this taxon appears to be complex, but rather uniform with regard to major compounds in most of the studied material with the exception of some extremely rare, aberrant chemotypes (Ohmura 2001, 2012).

In contrast, Dolichousnea trichodeoides (Vain. ex Motyka) Articus was not supported and exhibited substantial internal variation, with some strongly supported subclades on long stem branches, suggesting the presence of several lineages even within a single country (Japan; Ohmura 2002; Articus 2004; Truong et al. 2013a). This is another species that has only been reported from Africa, Asia and Oceania (Ahti et al. 2016; Aptroot 2016; Articus 2004; Galloway 2007; Jayalal et al. 2013; Ohmura 2002; Schumm \& Aptroot 2010; Stevens 1999; Swinscow \& Krog 1978, 1988; Truong et al. 2013a; Ohmura \& Kashiwadani 2018), but one unpublished accession (MN006813), deposited by M. L. Hale and J. Taylor in 2019, appears to be from California, although the geographic origin was not precisely indicated. That sequence formed part of a small, strongly supported subclade also including sequences from Japan and Taiwan (Fig. 7; Fig. S1). This taxon thus appears to represent an unresolved species complex, the various lineages rather well separated in the identity matrix (Fig. 11). Ohmura (2001, 2012) reported three chemotypes for D. trichodeoides, including salazinic or fumarprotocetraric acids or atranorin as major compounds. For one sequenced accession

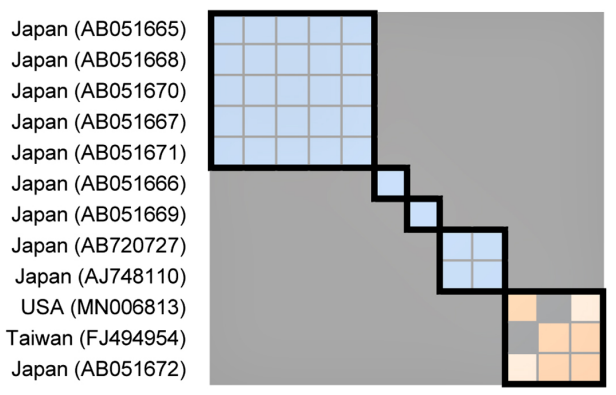

Figure 11. Pairwise identity matrix based on the ITS for the Dolichousnea trichodeoides clade. Dark grey = identity below $98.5 \%$; grey $=$ identity exactly $98.5 \%$; blue and beige = identity above $98.5 \%$. 
from Japan (AB051668), the chemotype (fumarprotocetraric acid) was given by Ohmura (2001), corresponding to the first diverging, larger subclade. Chemical data for the other specimens have apparently not been published, but should be available from the voucher material, so testing of a potential correlation between phylogeny and chemotypes should give further insight into this clade.

Considerable internal variation was also observed in the supported Dolichousnea longissima clade with samples from across the Northern Hemisphere (Figs 2, 7; Table S1; Fig. S1). The internal structure was largely reflected in a cascading backbone, without clearly discernable larger lineages. The corresponding identity matrix identified several subgroups at the $98.5 \%$ level, but pairwise comparison between samples across all subgroups also revealed partially high identity levels above $98.5 \%$ (Fig. 12). Thus, D. longissima apparently cannot currently be subdivided into more than one species based on ITS data, even if it exhibited considerable internal variation. Secondary chemistry may help to discern lineages, although it has not been reported for most of the sequenced specimens. The three most common chemotypes produce diffractaic, barbatic, or evernic acid as major compounds (Halonen et al. 1998; Ohmura 2001, 2012; Randlane et al. 2009). The diffractaic acid chemotype corresponds to D. longissima s.str. according to the type material (Ohmura 2001, 2012). Two sequenced Japanese samples for which the chemistry has been reported, AB051643 (barbatic acid major) and AB051645 (diffractaic acid major), cluster in distant portions of the clade.

Few data from other markers were available for Dolichousnea. IGS data for D. longissima (from Rolstad et al. 2013) also showed a high level of internal structure for that taxon (Fig. S3). Unfortunately, there was little overlap between the ITS and IGS data from the same study (Rolstad et al. 2013). Of the 48 samples with either ITS or IGS data, only ten had both markers. Based on these, the internal topology for D. longissima was largely congruent between both markers and did not exhibit supported conflict, although IGS provided a more resolved topology (Figs S1, S3). Due to a limited amount or lack of data, the other markers could not be compared with ITS.

\section{Usnea subgenus Neuropogon New Zealand clade.}

This large, unsupported clade of 150 terminals was almost exclusively composed of samples from New Zealand, with two exceptions: one unpublished sequence labeled $U$. aff. igniaria Motyka from Antarctica (DQ219307), deposited by K.-M. Kim and J.-S. Hur in 2005, and one unidentified sequence from Chile (KJ406284) from the study by Millanes et al. (2014). Both formed separate singletons likely representing separate species. Of the remaining 148 sequences, only nine were identified as GenBank deposits, representing $U$. ciliata and $U$. subcapillaris

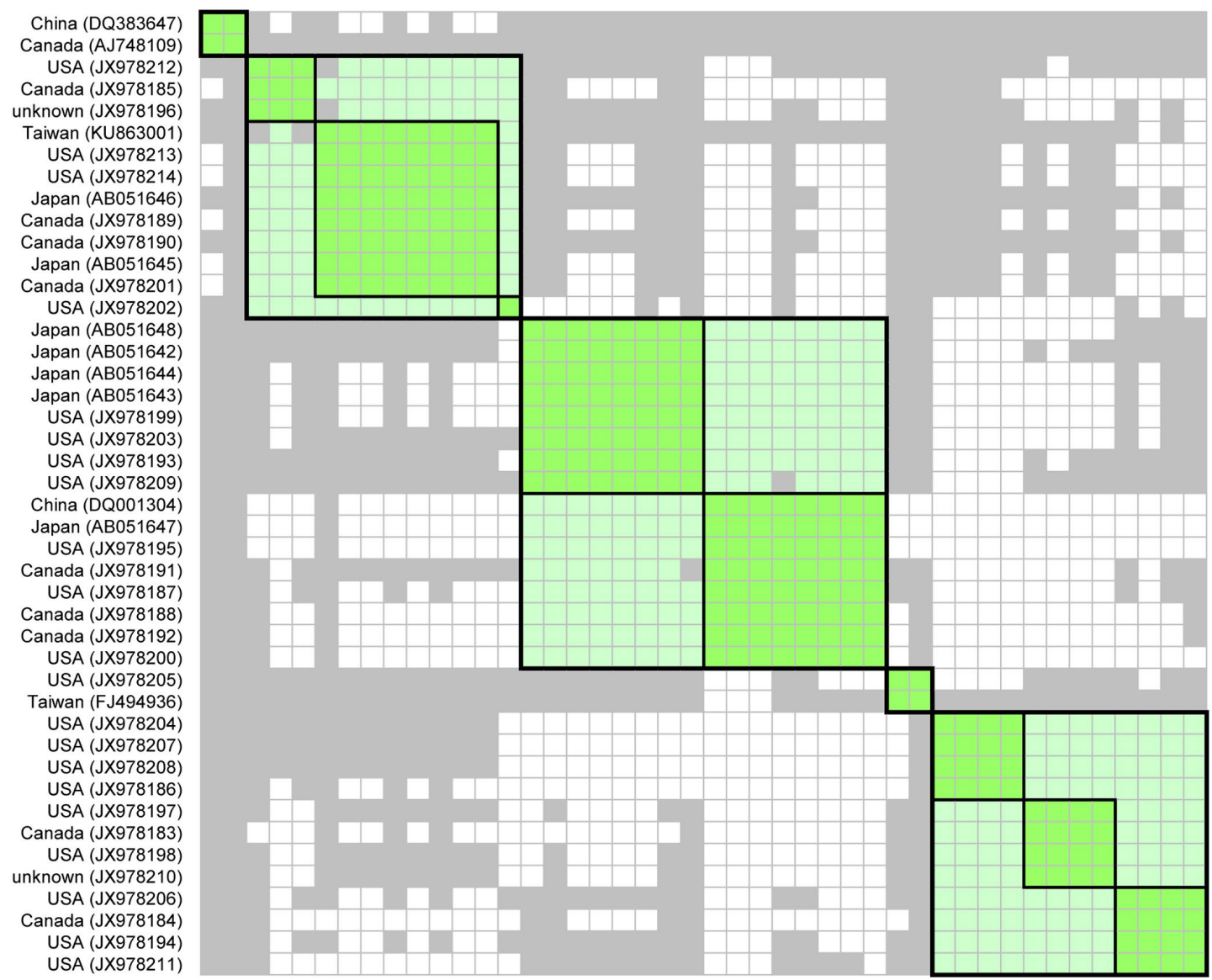

Figure 12. Pairwise identity matrix based on the ITS for the Dolichousnea longissima clade. Dark grey $=$ identity below $98.5 \%$; grey $=$ identity exactly $98.5 \%$; white and shades of green = identity above $98.5 \%$. 
(Wirtz et al. 2006, 2012; Lumbsch \& Wirtz 2011). The remaining 139 terminals almost exclusively stem from two studies (Buckley et al. 2014; Rafat et al. 2015). Of these, 18 specimens were identified in the corresponding papers, but left unidentified in the GenBank accessions.

Given this information, the entire clade can currently only be outlined provisionally (see Fig. 7; Fig. S1). Following three singletons as early diverging lineages, a paraphyletic grade of ten sequences (KM369335 through KM369241) included two subsequently identified as U. torulosa (Buckley et al. 2014; Rafat et al. 2015) and was here interpreted to represent that taxon. The grade contained an unsupported subclade of five sequences (KM369431 through KM369241), differing consistently in two substitutions in the ITS2, i.e. about $99.6 \%$ similarity with the other sequences and not sufficient to consider this a separate species. The first of these sequences (KM369431) included odd base calls (see above), accounting for the long branch. Another, strongly supported clade of five terminals (KM369197 through KM369435), containing one accession subsequently identified as U. torulosa (Buckley et al. 2014), differed consistently in five substitutions (99\% similarity) and was here considered a separate species ( $U$. aff. torulosa), as it fell elsewhere. A similar situation was found with five specimens identified as $U$. inermis (Buckley et al. 2014; Rafat et al. 2015), which fell into two larger, unsupported clades (Fig. 7; Fig. S1), the first considered U. inermis s.str. and the second $U$. aff. inermis. The first clade of 27 accessions (KM369276 through KM369332) was internally rather uniform, but contained several sequences with odd base calls (see above; Table S1). The second clade formed two subclades, each with one sequence subsequently identified as $U$. inermis. The first, smaller subclade (KM369369 through KM369249) was sister to two specimens subsequently identified as U. pusilla (Buckley et al. 2014; Rafat et al. 2015), here labeled $U$. aff. pusilla (see below). They differed from the second, larger, rather uniform subclade (KM369279 through JX144643) consistently in six substitutions ( $98.8 \%$ similarity) and so the two subclades were here labeled $U$. aff. inermis 1 and $U$. aff. inermis 2 .

Three specimens of Usnea subcapillaris (Wirtz et al. 2006, 2012; Lumbsch \& Wirtz 2011) formed a strongly supported clade, whereas a fourth accession fell separate (U. aff. subcapillaris). The same applied to U. ciliata (Wirtz et al. 2006, 2012; Lumbsch \& Wirtz 2011), where a single accession originally identified as $U$. cf. ciliata did not cluster with the remaining samples, which formed a paraphyletic grade basal to U. subcapillaris (Fig. 7; Fig. S1), including also one originally unidentified sample (Buckley et al. 2014). Four accessions (JX144646 through KM369371) formed a fully supported clade on a long branch; one was subsequently identified as $U$. articulata (Rafat et al. 2015) and was here labeled $U$. aff. articulata 4 , although this lineage was obviously not related to $U$. articulata (see below).

The remaining accessions largely represented three names subsequently identified in the corresponding studies (Buckley et al. 2014; Rafat et al. 2015): U. ciliifera,
U. pusilla, and U. xanthopoga. Twenty accessions (JX144648 through KM369173) formed an unsupported clade containing five terminals subsequently labeled $U$. ciliifera. These contained a well-unsupported subclade (88\%) of eight accessions (KR091723 through KM369173), two with odd base calls (see above) on long branches (Fig. 7; Fig. S1). This latter subclade had two consistent substitutions and one indel $(99.4 \%$ similarity) and so the two groups were labeled $U$. ciliifera 1 and $U$. ciliifera 2 . The Usnea pusilla clade (KM369188 through JX144650), including a single, subsequently identified accession (Rafat et al. 2015), was well-supported and internally variable, partly due to accessions with odd base calls, but did not exhibit supported internal structure. The large U. xanthopoga clade (KM369401 through KM369408) included two sequences subsequently identified with that name (Rafat et al. 2015). This clade was more or less supported (74\%) and included some partly supported subclades, in particular one consisting of three accessions (KM369248, KM369436, JX144644) with three consistent substitutions, and another of nine accessions (KM369409 through KM369408) with two consistent substitutions, all in the ITS1 (File S1). In addition to these subsequently identified or provisionally identified clades, the New Zealand clade of subgenus Neuropogon contained four separate lineages that remained unidentified (Fig. 7; Table S1; Fig. S1).

Unfortunately, few data are available on other markers for this clade. Only IGS and RPBI covered specimens of this clade, whereas data on other markers all corresponded to the Neuropogon core clade (see below). Both IGS and RPB1 data (Lumbsch \& Wirtz 2011; Wirtz et al. 2012) placed accessions identified as U. ciliata and U. subcapillaris in a strongly to fully supported clade within the supported Neuropogon core clade (Fig. S3), thus supporting the close relationship of the New Zealand clade with the species in the core clade, but leaving open the question whether both groups indeed form sister clades, as suggested by the unsupported ITS topology.

Walker (1985) reported six species corresponding to subgenus Neuropogon for New Zealand: U. acromelana, $U$. antarctica, U. ciliata, U. pseudocapillaris, U. sphacelata, and U. subcapillaris. Three of these (U. acromelana, $U$. antarctica, U. sphacelata) belong to the Neuropogon core clade (see below) and have not yet been confirmed to occur in New Zealand based on sequence data. Thus, of the seven names used to identify lineages in this clade (U. ciliata, U. ciliifera, U. inermis, U. pusilla, U. subcapillaris, U. torulosa, U. xanthopoga), only two agreed with Walker (1985) and one (U. pseudocapillaris) was missing, possibly corresponding to one of the unnamed or provisionally named clades here. This suggests that subgenus Neuropogon in New Zealand requires a thorough phylogenetic revision. Both $U$. inermis and $U$. torulosa were treated by Walker (1985) as somewhat similar to subgenus Neuropogon, but not related to the latter. If the identifications given by Buckley et al. (2014) and Rafat et al. (2015) are correct, then these taxa belong in subgenus Neuropogon. Usnea xanthopoga was mentioned by Walker (1985), but referred to subgenus Usnea and, based 
on the available data, it also belongs in subgenus Neuropogon. Usnea ciliifera and U. pusilla were not treated by Walker (1985) and not related to the Neuropogon group by Galloway (2007). Overall, the New Zealand clade of subgenus Neuropogon thus appears to include some species that do not share the typical characteristics of the Neuropogon core clade (see below).

Usnea subgenus Neuropogon core clade. Although containing a much larger number of terminals (446), this clade was genetically more uniform compared to the New Zealand clade (Fig. 7; File S1; Fig. 10). Species in this clade are generally characterized by annulate black pigmentation along the branches (Fig. 1C) and black apothecial discs (Walker 1985; Ohmura 2002; Ohmura \& Kanda 2004; Articus 2004; Wirtz et al. 2006, 2008, 2012; Seymour et al. 2007). Notably, this clade did not contain a single accession from New Zealand and was largely restricted to Antarctica and South America, with the exception of the more widely distributed $U$. lambii and $U$. sphacelata.

More than half of the Neuropogon core clade (247 accessions) was comprised of the species pair $U$. antarctica (sorediate) and U. aurantiacoatra (apotheciate), both with norstictic and salazinic acids as major compounds (Walker 1985). These accessions stem from a large number of studies (Articus 2004; Ohmura \& Kanda 2004; Kim et al. 2006; Seymour et al. 2007; Lumbsch \& Wirtz 2011; Schmull et al. 2011; Wirtz et al. 2006, 2012; Park et al. 2015; Cao et al. 2015, 2018; Lagostina et al. 2018; Canini et al. 2020), including numerous apparently unpublished sequences deposited by K.-M. Lim \& J.-S. Hur in 2005, N. Wirtz in 2006, H. Li \& Q.-M. Zhou in 2012, and S. Cao in 2015. Among Usnea s.lat., this is by far the best studied species-level clade in terms of taxonomy, phylogeny, ecology, and distribution. The strongly supported clade was comparatively uniform regarding the ITS, with some unsupported internal structure and no resolution into clades corresponding to either name, suggesting this species pair to represent a single taxon (Seymour et al. 2007; Wirtz et al. 2012). Data from the IGS (Wirtz et al. 2012) did not include sorediate forms (U. antarctica), but the apotheciate forms ( $U$. aurantiacoatra) formed several smaller, partly supported, nested subclades with $U$. acromelana nested within on a long, strongly supported branch (Fig. S3). RPB1 data (Seymour et al. 2007; Wirtz et al. 2012) showed a similar pattern as with ITS with $U$. antarctica samples intermingled with $U$. aurantiacoatra (Fig. S5). Using a RADseq approach, Grewe et al. (2018) demonstrated the separation of $U$. antarctica and $U$. aurantiacoatra into two strongly supported clades, suggesting the presence of two closely related species that ITS, IGS and $R P B 1$ were not able to resolve. This confirmed findings by Lagostina et al. (2018) using microsatellite markers, although in the latter study, some apotheciate specimens clustered with the sorediate $U$. antarctica, suggesting a more complex situation in which two species can be distinguished, one persistently fertile (apotheciate) and one usually sterile (sorediate) and sometimes fertile (apotheciate). This would mirror results from other works, such as the classic study by Kroken \& Taylor (2001) on the Letharia columbiana / L. vulpina species pair, which demonstrated the existence of six lineages, the sorediate ones sometimes also producing apothecia.

Wirtz et al. (2008) and Lumbsch \& Wirtz (2011) studied the Usnea perpusilla complex, resolving it into five species, besides $U$. perpusilla also distinguishing U. lambii, U. messutiae Wirtz \& Lumbsch, U. pallidocarpa Wirtz \& Lumbsch and U. ushuaiensis. The five taxa formed three groups in the ITS-based tree, one consisting of $U$. ushuaiensis, one representing the $U$. perpusilla-lambii aggregate, and one the U. messutiae-pallidocarpa aggregate. The $U$. ushuaiensis clade was not supported, likely due to an unidentified singleton sequence (AY251434) on a long branch nested within (Thell et al. 2004). A supported subclade included three accessions (EF492213, EF492167, EF492182), differing from the other sequences in three consistent substitutions. One of these accessions was regarded as 'potential recombinant' by Wirtz et al. (2008), but the isolated positions of the three substitutions ( $99.4 \%$ similarity) and the good support for this clade did not support this assumption; rather, it was here considered an infraspecific lineage. Medullary secondary chemistry in this clade is comparatively simple, lacking substances or containing psoromic acid as major and/or the dibenzofurane hypostrepsilic acid chemosyndrome as minor (Walker 1985; Elix et al. 2007; Lumbsch \& Wirtz 2011; Lumbsch et al. 2011). Wirtz et al. (2008) found a lack of correlation between clades and secondary chemistry, but unfortunately did not report the exact chemistry for each terminal.

Usnea perpusilla and $U$. lambii were not resolved as reciprocally monophyletic clades in the ITS and instead formed several supported and unsupported subclades (Fig. 7; Fig. S1). The same clades were also recovered in the IGS, although with higher support (Fig. S3). The two taxa are distinguished by their reproductive mode, U. perpusilla producing apothecia and U. lambii soredia. Wirtz et al. (2008) resolved $U$. perpusilla as paraphyletic relative to $U$. lambii, but this topology was likely caused by the inclusion of $R P B 1$, in addition to ITS and IGS. Our analysis showed that $R P B 1$ behaved erratically for this complex and other species in the Neuropogon core clade, mixing accessions of various taxa in various shallow grades and clades, an indication of the formation of paralogs (Fig. 13; Fig. S5). Lumbsch \& Wirtz (2011) recovered U. perpusilla and $U$. lambii as reciprocally monophyletic, but this was an artifact of limited sampling, representing one subclade each of the complex assemblage evident when joining all available data. Based on both ITS and IGS data, the situation in $U$. perpusilla versus $U$. lambii appears similarly complex as in Letharia columbiana (Nutt.) J.W. Thomson versus L. vulpina (L.) Hue (Kroken \& Taylor 2001; see above). Based on the identity matrix from the ITS data (not shown), the differences between the various subclades were very shallow with most pairwise similarity values at or above $98.5 \%$. However, considering the results from microsatellite and RADseq markers in the much shallower $U$. antarctica-aurantiacoatra complex (see above), it is 


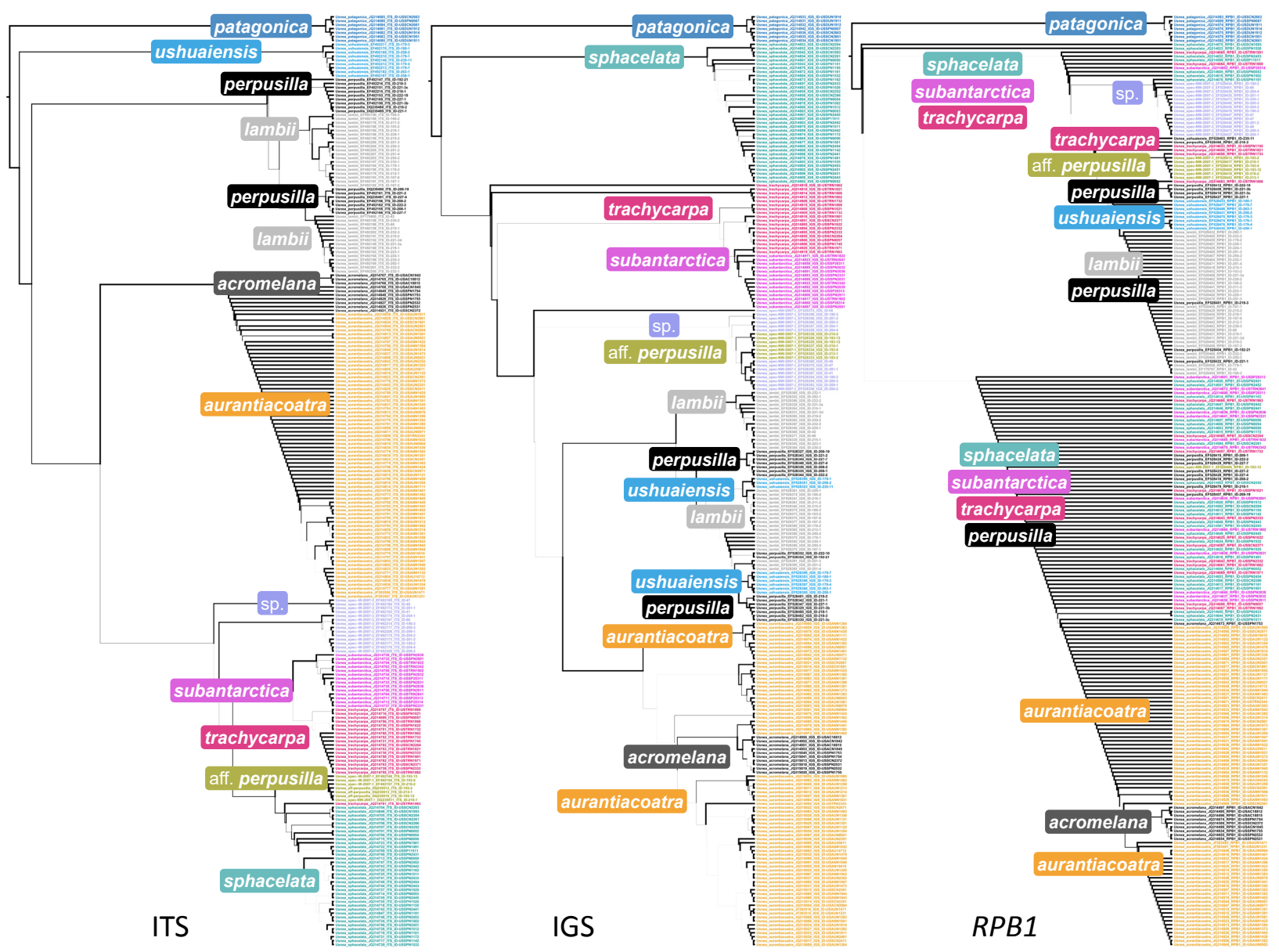

Figure 13. Comparison of topologies in the Neuropogon core clade between the ITS, IGS and RPB1 (cladograms). Each color corresponds to an accepted species. $R P B 1$ provided limited resolution or coherence for some species, but accessions of others were distributed across the two main clades.

possible that each of the clades formed in the U. perpusilla-lambii complex represents a separate species.

Usnea messutiae formed two unsupported subclades in the ITS, associated with a strongly supported clade representing U. pallidocarpa (Fig. 7; Table S1; Fig. S1), the latter including additional accessions from Wirtz et al. (2006). IGS data exhibited a partly conflicting topology. The second $U$. messutiae subclade was mirrored by a strongly supported subclade in the IGS, whereas the first subclade was not only dissected into a grade, a strongly supported subclade, and one singleton in the IGS, but the $U$. messutiae grade also included two accessions of U. pallidocarpa with identical sequences (Fig. S3), one of the rare instances of supported conflict between ITS and IGS data in Usnea. In contrast, RPB1 reflected both species well in two unsupported, homogeneous clades (Fig. S5).

The remaining five species in the Neuropogon core clade formed comparatively homogeneous groupings: $U$. patagonica and $U$. subantarctica were strongly supported each in monophyletic clades, $U$. sphacelata clustered in an unsupported clade, and $U$. trachycarpa and $U$. acromelana in paraphyletic grades, one basal to $U$. subantarctica, that relationship without support, and the other basal to the $U$. antarctica-aurantiacoatra complex, that relationship being strongly supported (Fig. 7; Fig. S1). With IGS, U. patagonica and U. sphacelata formed supported clades, whereas $U$. trachycarpa was again rendered paraphyletic and the $U$. subantarctica clade was not supported; U. acromelana formed a strongly supported clade, but was nested within the $U$. antarctica-aurantiaca complex (Fig. S3). As mentioned, RPB1 exhibited an erratic topology for taxa in this and the previous group, joining $U$. sphacelata, $U$. subantarctica, and $U$. trachycarpa in a homogeneous grade also including isolated accessions of U. pallidocarpa, U. perpusilla, and U. ushuaiensis (Fig. 13). Another, unsupported shallow clade was composed of accessions of $U$. aurantiacoatra, $U$. lambii, U. perpusilla, U. sphacelata, and U. ushuaiensis. A third group formed a shallow grade including $U$. acromelana, the $U$. antarctica-aurantiacoatra complex, and a single accession of $U$. sphacelata. Only $U$. patagonia formed a fully supported clade (Fig. S5). Given particularly the data of the ITS and IGS, there was thus at least partial support for U. acromelana, U. patagonica, U. sphacelata, $U$. subantarctica, and $U$. trachycarpa to form well-delimited species.

We detected only two instances of potentially misidentified accessions in the entire Neuropogon core clade (Table S1), both identified as $U$. subantarctica, but falling within U. trachycarpa. Five further accessions labeled U. sphacelata (Ohmura \& Kanda 2004; Seymour et al. 2007; Schmull et al. 2011) belonged in the U. perpusilla-lambii complex which was only subsequently defined by Wirtz et al. (2008) and Lumbsch \& Wirtz (2011). 
Two singleton sequences identified as U. trachycarpa (AJ748103, JQ314761; Articus 2004; Wirtz et al. 2012) fell at the base of the $U$. sphacelata clade and one of these (AJ748103) had odd base calls.

Usnea s.str. - Singletons. A total of 14 names were represented in the ITS-based tree by singleton clades only. These are considered putatively correct identifications representing separate species. They include $U$. angulata Ach. (JQ837291), U. aspera (Eschw.) Vain. (MF669805), U. aurantiaciparvula A. Gerlach \& P. Clerc (KY021902), U. cirrosa Motyka (KY021903), U. ghattensis G. Awasthi (KY021914), U. halei P. Clerc (MG252375), U. intumescens (AB051641), U. meridionalis Zahlbr. (KY021919), U. merrillii (AB051649), U. aff. sanguinea Swinscow \& Krog (MW267168), U. shimadae Asahina (FJ494952; deposited as Usnea sp.), U. sinensis Motyka (FJ494953, deposited as Usnea sp.), U. subglabrata Truong \& P. Clerc (JQ837312, deposited as Usnea sp. 6), and U. subrubicunda P. Clerc (JQ837332; Ohmura 2002; Shen et al. 2012; Truong et al. 2013a, 2016; Nadel 2016; Gerlach et al. 2017, 2019a).

Among these, two taxa are common and presumably widespread, namely $U$. angulata (Fig. 14) and U. merrillii (Marcano et al. 1996; Herrera-Campos et al. 1998; Elix \& McCarthy 1998, 2008; Stevens 1999; Ohmura 2002; Galloway 2007; Lin 2007; Singh \& Sinha 2010; Truong et al. 2013b; Herrera-Campos 2016; Sipman \& Aguirre-C. 2016; Truong \& Clerc 2016; Rodríguez-Flakus et al. 2016; Ohmura \& Kashiwadani 2018; Bungartz et al. 2018; Esslinger 2019; Lücking 2020c), and hence their sparse representation by ITS sequence data was surprising. Usnea angulata, which is particularly common in the Americas (Fig. 14), formed an early diverging lineage in our phylogeny, although this topology was not supported (Fig. 7; Table S1; Fig. S1). Usnea merrillii formed a separate lineage near $U$. clerciana Truong, but that relationship was also not supported. Another presumably widespread species is U. shimadae, reported from Mexico and Taiwan (Clerc 2007; Ohmura 2001, 2012; Shen et al. 2012; Herrera-Campos 2016). The only available sequence, that clustered with a supported, unidentified sequence from China (MT261826), was from Taiwan and corresponds to the taxon geographically, whereas the reports from Mexico need to be further investigated.

Five species are either widespread in the Americas or found across North or South America: U. aspera, U. cirrosa, U. halei, U. meridionalis, and U. subrubicunda (Hekking \& Sipman 1988; Marcano et al. 1996; Clerc \& Herrera-Campos 1997; Herrera-Campos et al. 2001; Calvelo \& Liberatore 2002; Clerc 2007; Truong et al. 2011, 2013a; Truong 2012; Herrera-Campos 2016; Truong \& Clerc 2016; Gerlach et al. 2017, 2019a; Rodríguez-R. et al. 2017; Esslinger 2019; Lücking et al. 2020c). For these taxa, the paucity of sequence data was surprising. Usnea aspera clustered with $U$. aff. cornuta (not representing that species, see below) from Brazil; U. cirrosa fell in the vicinity of $U$. cladocarpa and $U$. halei close to $U$. flammea Stirt., both relationships without support; U. meridionalis formed an unsupported clade with $U$. durietzii and $U$. glabrata; and $U$. subrubicunda was found, without support, basal to the U. barbata-dasopoga-wasmuthii clade (Fig. 7; Table S1; Fig. S1).

The remaining six species included two recently described taxa, U. subglabrata from Bolivia (Truong \& Clerc 2016) and U. aurantiaciparvula from Brazil (Gerlach et al. 2017), and four species with more restricted distribution in Africa and Asia, namely U. ghattensis, $U$. intumenscens, $U$. aff. sanguinea, and $U$. sinensis (Ohmura 2002; Lin 2007; Singh \& Sinha 2010; Shen et al. 2012; Gerlach et al. 2017; Ohmura \& Kashiwadani 2018). Usnea ghattensis fell close to $U$. articulata, while $U$. intumescens clustered with support with $U$. bismolliuscula and $U$. sinensis with $U$. nipparensis (Fig. 7; Table S1; Fig. S1). The accession originally identified

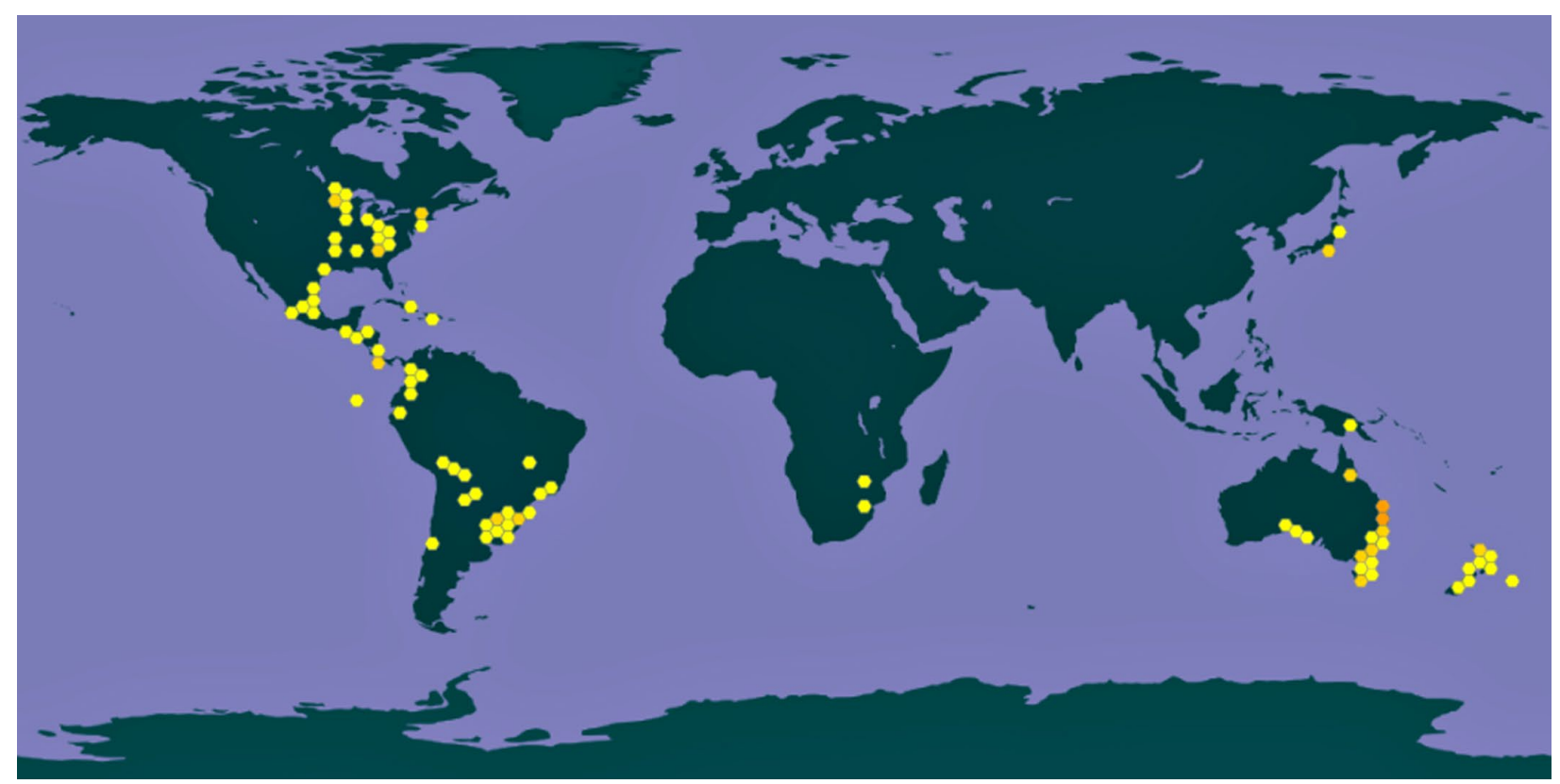

Figure 14. World distribution of Usnea angulata according to the Global Biodiversity Information Facility, GBIF [https://www.gbif.org/species/2606124], fitting well with published records in taxonomic revisions. 
as $U$. aff. sanguinea (Nadel 2016) formed a singleton clade unrelated to $U$. erinacea with which it has been synonymized (Clerc 2004, 2007, 2011a; see below). However, the voucher specimen, while lacking soredia, does not produce apothecia and has a deviating chemistry, so this identification is preliminary.

No IGS data were available for any of the singletons. In RPB1, two taxa, U. aurantiaciparvula and U. ghattensis, also formed singletons, whereas two accessions of $U$. cirrosa formed a paraphyletic grade at the base of U. cornuta s.str. (Fig. S5).

Usnea s.str. - Monophyletic species-level clades. A total of 30 taxa were recovered as monophyletic and internally more or less homogeneous and could be considered well-defined species. These are U. acanthella, U. aranea Truong \& P. Clerc, U. cavernosa, U. chilensis Motyka, $U$. cladocarpa, U. clerciana, U. densirostra Taylor, U. dodgei Motyka, U. durietzii, U. flammea, U. flavocardia Räsänen, U. fleigiae A. Gerlach \& P. Clerc, U. grandisora Truong \& P. Clerc, U. grandispora A. Gerlach \& P. Clerc, $U$. hirta, U. macaronesica P. Clerc, U. malmei Motyka, U. masudana Asahina, U. nidifica Taylor, U. nipparensis, U. oreophila A. Gerlach \& P. Clerc, U. pangiana, U. parvula Motyka, U. pseudogatae, U. rubriglabrata Truong \& P. Clerc, U. silesiaca, U. subaranea Truong \& P. Clerc, U. subdasaea Truong \& P. Clerc, U. subparvula, and U. wasmuthii (Fig. 7, Table S1, Fig. S1).

Usnea acanthella, described from Peru (Lamb 1939) and represented by various accessions from Ecuador and Peru (Wirtz et al. 2006, 2012), was one of two neuropogonoid species falling outside the Neoropogon clade (Wirtz et al. 2006). Usnea aranea was recently described based on a non-sequenced specimen from Bolivia as type, whereas the sequenced specimens originated from Ecuador and Peru (Truong et al. 2013a; Truong \& Clerc 2016). Given the complexity of species delimitations in the genus Usnea, this could cause problems unless a sequenced epitype is being designated. Usnea cavernosa was originally described from North America (Tuckerman in Agassiz 1850) and the fully supported clade, including specimens from the USA and Switzerland (Mark et al. 2016a), was consistent with the inferred distribution of the species (see below). As the epithet implies, U. chilensis was originally established based on material from Chile (Motyka 1938), but was here represented by two sequences from southern Brazil (Gerlach et al. 2019a). A similar situation applied to U. cladocarpa, a taxon described from Brazil (Fée 1825), with two sequences from Costa Rica (Gerlach et al. 2017). Given that presumably widespread neotropical or American species in other genera have recently been shown to represent complexes of species with more restricted distribution, as in the case of Neoprotoparmelia multifera (Nyl.) Garima Singh, Lumbsch \& I. Schmitt (Santos et al. 2019). Thus, such cases in the genus Usnea need to be further explored by attempting to collect samples from the type regions.

The recently established Usnea clerciana, a putative Galapagos endemic, is an example of a taxon including sexual and asexual morphs in a single species from the onset (Truong \& Clerc 2016). The four ITS accessions were rather uniform, but the case of $U$. antarctica vs. $U$. aurantiacoatra (see above) shows that species pairs may not necessarily be resolved with the ITS marker. The saxicolous, apotheciate species $U$. densirostra Taylor, described from Uruguay, was represented by three accessions from nearby southern Brazil (Gerlach et al. 2017, 2019a). Its sorediate counterpart, U. amblyoclada (Müll. Arg.) Zahlbr, also saxicolous and known from the same area (Rodriguez et al. 2011a), has not yet been sequenced. Usnea dodgei and $U$. durietzii represent cases similar to $U$. chilensis and $U$. cladocarpa, whereas $U$. dodgei was described from Costa Rica (Motyka 1938), with three accessions from Brazil (Gerlach et al. 2019a), U. durietzii represents a neuropogonoid taxon originally described from Chile (Motyka 1938), with two ITS accessions from Peru (Wirtz et al. 2006).

Phylogenetic inference for Usnea flammea, forming a well-supported clade of several accessions from Macaronesia and western Europe (Kelly et al. 2011; Saag et al. 2011; Schoch et al. 2012; Gerlach et al. 2019a; Marthinsen et al. 2019), was well in line with its type originating from Portugal (Stirton 1881). The situation was the opposite for $U$. flavocardia, described from Chile (Räsänen 1936), but all ITS accessions originating from Europe and North America (Kelly et al. 2011; Saag et al. 2011; Schoch et al. 2014; Millanes et al. 2014; Araujo 2016). The two presumed North American accessions are unpublished, deposited by M. L. Hale and J. Taylor in 2019, and did not have specific voucher information, so their exact origin remains unclear. A further accession from New Zealand (KJ406280) identified with this name (Millanes et al. 2014) fell outside the flavocardia clade, but clustered with an unidentified lineage from New Zealand (Buckley et al. 2014). Usnea fleigiae, U. grandisora, and $U$. grandispora are recently established species from South America (Truong \& Clerc 2016; Gerlach et al. 2017). Usnea grandisora was described from Galapagos and also reported from Venezuela (Truong \& Clerc 2016), whereas the available ITS sequences are from Brazil (Gerlach et al. 2017), so the status of this taxon remains unclear.

Usnea hirta is one of the presumably most widespread species in the genus with reports from all major areas in North and South America, Europe, Africa, Asia, and Oceania (Mies 1989; Halonen et al. 1998; Elix \& McCarthy 1998, 2008; Fos \& Clerc 2000; Calvelo \& Liberatore 2002; Articus et al. 2002; Bjerke et al. 2006; Clerc 2007; Randlane et al. 2009; Kelly et al. 2011; Saag et al. 2011; Shrestha et al. 2012; Noer et al. 2013; Santiago et al. 2013; Myllys et al. 2014; Burkin \& Kononenko 2015; Shukla et al. 2015; Herrera-Campos 2016; Paliya et al. 2016; Gagarina et al. 2017; Galinato et al. 2017, 2018; Bungartz et al. 2018; Esslinger 2019). All accessions in this strongly supported clade were from Europe (Articus et al. 2002; Kelly et al. 2011; Saag et al. 2011; Millanes et al. 2014; Araujo 2016). It is unclear whether this taxon has simply not been sequenced from other areas, but given the geographic coverage of ITS accessions for the genus and the documented main distribution of $U$. hirta in North 
America and Europe (Fig. 15), a possible explanation is that $U$. hirta is a holarctic taxon, absent from the tropics and the Southern Hemisphere, and has simply not yet been sequenced in its North American and temperate Asian range.

Of the remaining species, six were also recently established, namely $U$. macaronesica from Portugal (Clerc 2006), with two accessions from that country (Millanes et al. 2014), the saxicolous $U$. oreophila from Brazil (Gerlach et al. 2019b), U. rubriglabrata from Peru (Truong et al. 2013a; Truong \& Clerc 2016), U. subaranea from Colombia (Truong \& Clerc 2016), U. subdasaea from Galapagos (Truong et al. 2011, 2013a), and U. subparvula from Brazil (Gerlach et al. 2017). Usnea macaronesica clustered with the singleton $U$. subglabrata from Bolivia (see above) in a fully supported clade, but both were separated by seven consistent substitutions (File S3). When describing U. subglabrata, Truong \& Clerc (2016) did not discuss the new species with $U$. macaronesica, a species established only ten years prior (Clerc 2006), although both are remarkably similar. One would be tempted to subsume $U$. subglabrata as synonym under $U$. macaronesica, but the notable differences in the ITS, together with the geographic distance of the samples, suggest that this is a clade undergoing cryptic speciation.

Usnea rubriglabrata is another case where a species may have been based on a non-sequenced type specimen with sequences possibly originating from other specimens. Truong \& Clerc (2016) gave Truong 1877 as holotype and Truong 1625 and Truong 1894 as paratypes, whereas the sequenced vouchers were identified as isolate 2 (JQ837320) and isolate 135 (KP668968), so there is no way of knowing whether one of these corresponded to the holotype or not. In the case of $U$. subaranea, based on type material from Colombia (Truong \& Clerc 2016), the two ITS accessions were definitely non-type material from Ecuador and Peru (Truong et al. 2013a; Truong
\& Clerc 2016). Usnea subdasaea is one example of a species originally sequenced only from Galapagos, but with additional material reported from continental South America (Truong et al. 2011, 2013a), and later confirmed through sequence data from Brazil (Gerlach et al. 2019a). A deviating terpenoid chemotype originally identified as U. subdasaea (Truong et al. 2013a) clustered elsewhere in our ITS-based tree (JQ837328) and represents a separate lineage, as already stated by Truong et al. (2013a).

The identity of sequenced specimens identified as Usnea malmei is most certainly incorrect. The pendulous, sorediate species with conspicuous annular cracks and elongate pseudocyphellae, producing norstictic and salazinic acid and the unknown substances UP1 and UP2, was described from Brazil (Motyka 1938) and has since been reported from Mexico (Herrera-Campos et al. 1998; Herrera-Campos 2016), the northern Andes (Truong et al. 2013b) and Argentina (Calvelo \& Liberatore 2002). Yet, the four available sequences all stem from Taiwan. Two unpublished sequences (FJ494937, FJ494938) were deposited by Y.-M. Shen et al. in 2008 and two additional accessions (KU863002, KU863003) originated from a DNA barcoding approach (Greenwood et al. 2016), who apparently took up the identification as $U$. malmei based on these previously deposited, unpublished and almost certainly mislabeled sequences. This is an exemplar case of a 'snowballing effect' in DNA barcoding, where initially misidentified sequences caused subsequently matching depositions to perpetuate this error (Gilks et al. 2002). The sequenced samples in this case appear to represent an Asian endemic, as suggested by their nested placement within a larger, unsupported clade of Asian lineages (Fig. 7; Fig. S1).

In contrast, the following five Asian species were all represented by ITS accessions either from the type region or at least near the type region: U. masudana, described from Taiwan (Asahina 1970) and with sequences from that

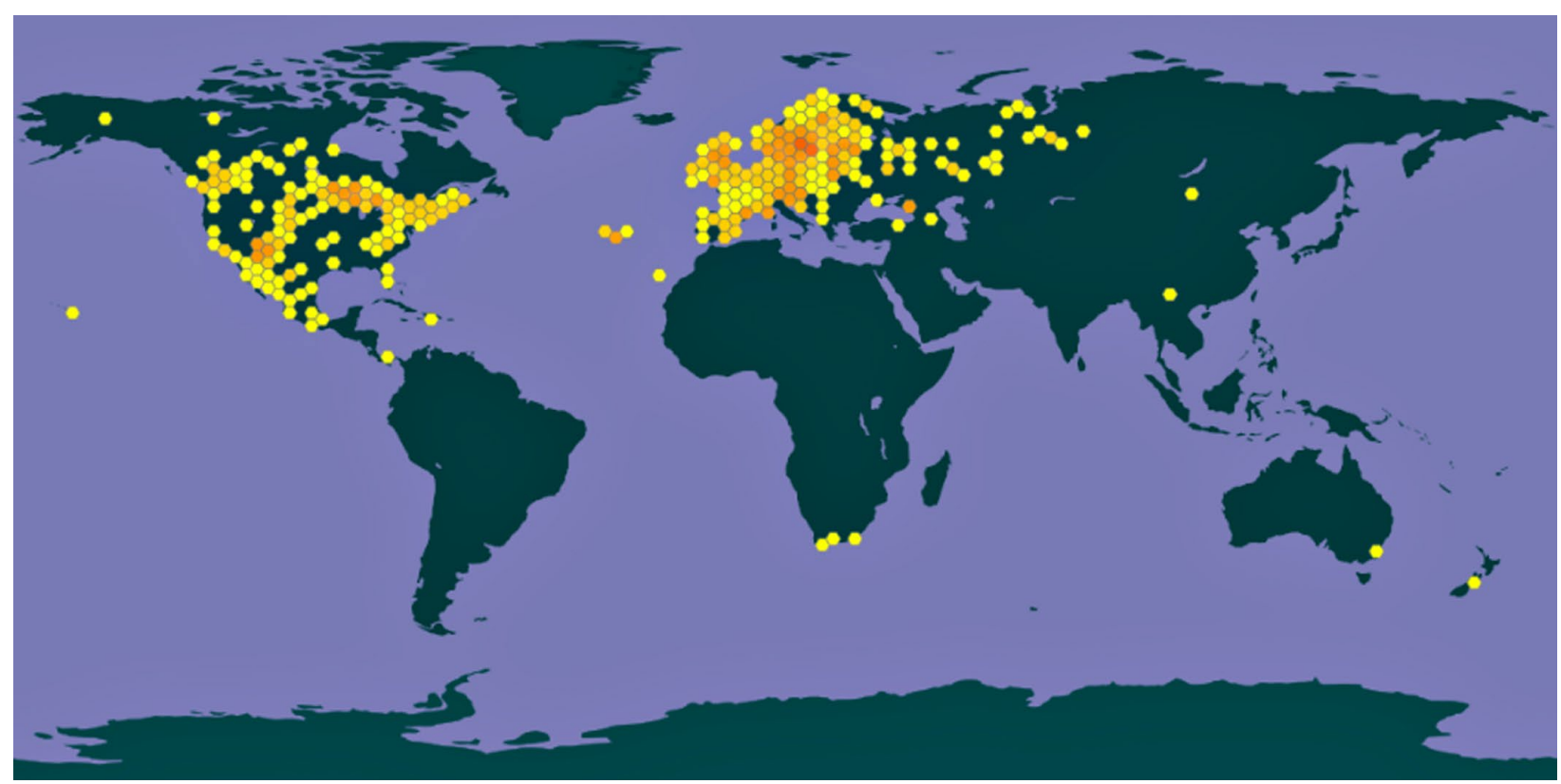

Figure 15. World distribution of Usnea hirta according to the Global Biodiversity Information Facility, GBIF [https://www.gbif.org/species/2606030]; the tropical and Southern Hemisphere records are not that species. 
region (Shen et al. 2012); U. nidifica, described from Oceania (Norfolk Island; Taylor 1847) and with two unpublished accessions from Taiwan; U. nipparensis, described from Japan (Asahina 1956), with accessions from Japan (Ohmura 2002, 2011) and unpublished sequences from China; U. pangiana, described from India (Stirton 1883), with sequences from Japan (Ohmura 2002) and China (unpubl.), and U. pseudogatae Asahina, described from Taiwan (Asahina 1970) and with sequences from the same area (Shen et al. 2012). In the cases of U. masudana and $U$. pseudogatae, the sequences were only identified in the corresponding paper (Shen et al. 2012), but not in the original depositions (see above). Usnea pangiana fell in a clade with several other sequences on long branches, including three additional unpublished accessions, deposited by S. Guo et al. in 2019 and one sequence under the name $U$. pangiana (AB051653; Ohmura 2002), but apparently not representing that species in the strict sense. This clade requires further study.

The remaining three species were historically described from the Americas or Europe: U. parvula from Argentina (Motyka 1938), with accessions from southern Brazil (Gerlach et al. 2017); U. silesiaca, from Poland (Motyka 1930), with sequences from Portugal and North America (Araujo 2016; Mark et al. 2016a) and an apparently misidentified outlier from Ecuador (Truong et al. 2013a); and U. wasmuthii Räsänen, described from Estonia (Räsänen 1931). The latter formed a more or less coherent clade of
34 accessions, including several from the type region, but was akin to a species complex, with considerable internal structure (Fig. 7; Fig. S1).

Data for other markers were available for some of these species, mostly $R P B 1$ and $M C M 7$, but also IGS, $T U B 2$, and RPB2 (Lumbsch \& Wirtz 2011; Saag et al. 2011; Wirtz et al. 2012; Mark et al. 2016a; Araujo 2016; Gerlach et al. 2017, 2019a). IGS data of four species rendered three of these monophyletic, although with internal structure, namely U. acanthella, U. cavernosa, and U. silesiaca. Usnea wasmuthii formed a paraphyletic grade and had one conflicting sequence (was-03) clustering with $U$. subfloridana (Fig. 16; Fig. S3). Data of TUB2 were available for six taxa, with two singletons (U. dasaea, U. flavocardia), two monophyletic clades lacking support and with considerable internal variation (U. cavernosa, U. hirta), and two polyphyletic assemblages, suggesting the potential formation of paralogs (U. silesiaca, U. wasmuthii; Fig. 16; Fig. S4).

$R P B 1$ offered data for 13 taxa, six of them singletons (U. cladocarpa, U. densirostra, U. fleigiae, U. grandispora, U. parvula, U. subparvula). Five further taxa resulted monophyletic and internally homogeneous, namely $U$. acanthella, U. erinacea, U. flammea, U. hirta, and U. silesiaca (Fig. S5). Usnea cavernosa exhibited some internal variation, whereas $U$. wasmuthii formed a paraphyletic grade with internal variation (Fig. S5). The situation for $R P B 2$ was different; of the three taxa with

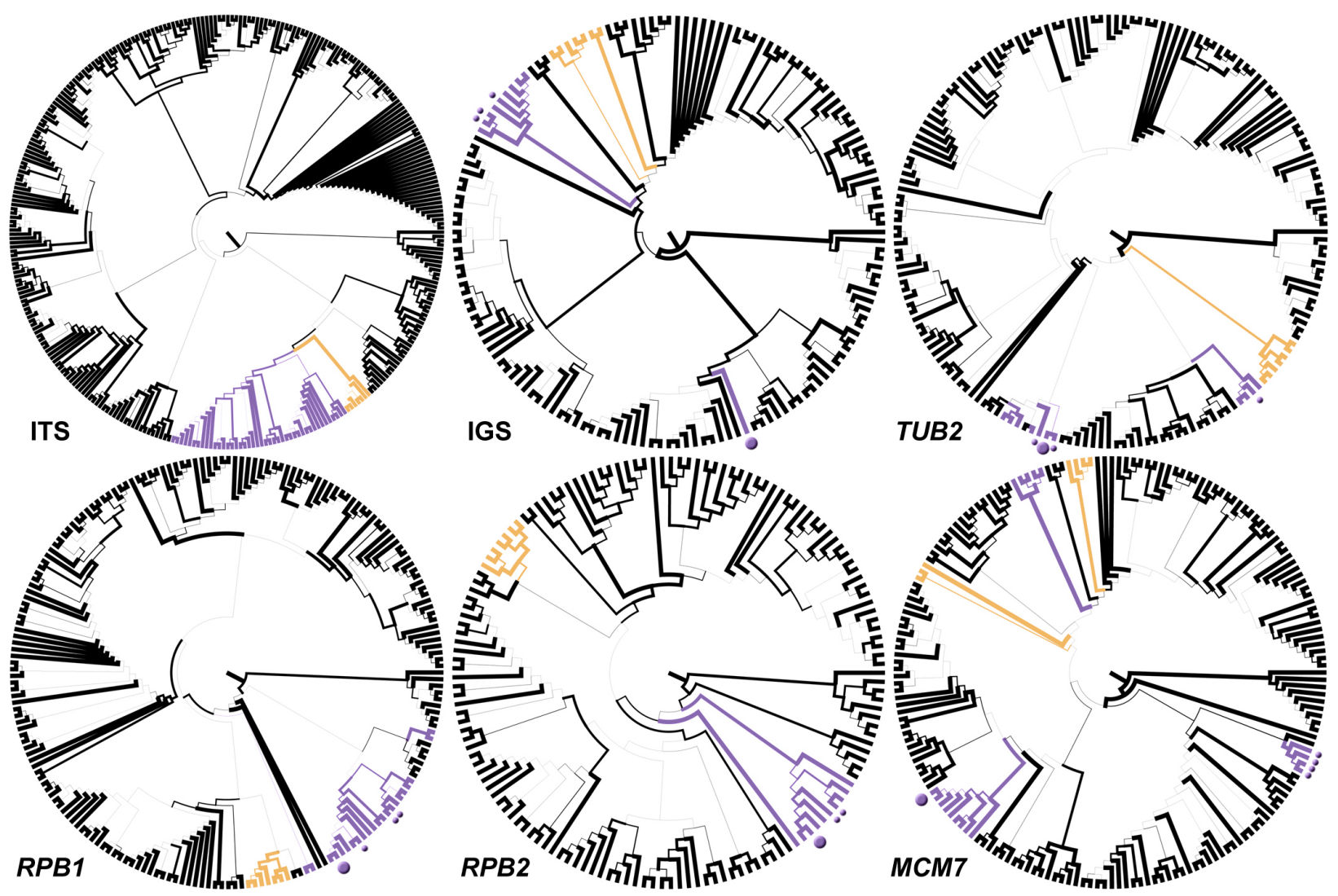

Figure 16. Comparison of the phylogenetic structure in six markers of Usnea sect. Usnea including the two species $U$. cavernosa (beige) and $U$. wasmuthii (purple). Both in TUB2 and MCM7 the formation of paralogs was evident. Selected corresponding specimens are marked with dots, including an outlier in the IGS (large dot) and one of the paralog clades in MCM7 (small dots). For detailed trees with labels, see Figs S1, S3-7. 
available data, only $U$. silesiaca was rendered monophyletic, although with internal variation (Fig. S6). Usnea cavernosa resulted polyphyletic, suggesting the formation of paralogs, and U. wasmuthii formed a grade plus one outlier (was-08; Fig. 16).

For a total of 18 taxa, data for the MCM7 were available. One represented a singleton (U. flammea) and only one was recovered monophyletic and internally homogeneous (U. oreophila). Seven taxa exhibited considerably internal variation, often more so than with other markers, namely $U$. densirostra, U. dodgei, U. fleigiae, U. grandisora, U. hirta, U. parvula, and U. subparvula (Fig. S7). Two further taxa that were rendered monophyletic with the ITS, U. cladocarpa and $U$. dasaea, formed paraphyletic, heterogeneous grades with long internal branches. Finally, seven taxa appeared polyphyletic with $M C M 7$, suggesting the formation of paralogs, including $U$. cavernosa, U. chilensis, U. erinacea, U. grandispora, U. silesiaca, U. subdasaea, and U. wasmuthii (Fig. 16; Fig. S7).

Usnea s.str. - Polyphyletic or highly variable species complexes. A large number of names representing common and widespread species were either polyphyletic or resulted in highly variable clades in the ITS (and other markers), suggesting that potentially more than one species were involved in these cases. They are outlined and discussed in detail as follows.

Usnea articulata aggregate. A total of 12 sequences were deposited under this name in GenBank, mostly from Europe including Russia and one from Cameroon, plus an additional sequence from New Zealand (JX144646), submitted as Usnea sp., but in the corresponding paper identified as $U$. articulata (Rafat et al. 2015). Further sequences were reported from Africa (São Tomé and Príncipe) by Nadel (2016). The European sequences, together with five accessions from São Tomé and Príncipe, formed a monophyletic, unsupported clade next to $U$. ghattensis and two unidentified, unpublished sequences of unknown origin, but likely from South Korea, deposited by J.-S. Hur in 2011. Additional sequences from São Tomé and Príncipe clustered in an unsupported clade in the vicinity with two basally emerging lineages representing the unnamed Usnea sp. Afrom the same area (Nadel 2016).

Thus, Usnea articulata exhibited considerable phylogenetic variation. Seven practically identical sequences from Europe formed the backbone of the main clade and four additional sequences fell on long branches. These four sequences were among those determined as of low quality (see above), apparently the reason for this aberrant topology and the overall low clade support. We therefore considered this an artifact, perhaps caused by a lichenicolous fungus specific to this taxon, and as a consequence, regarded $U$. articulata s.str. as represented by the group formed by the seven homogeneous, European accessions, is in line with the origin of the type material. This is an example how sequence quality could affect topology and hence taxonomic interpretation if sequence data are not inspected carefully. The African samples, including 13 from São Tomé and Príncipe (Nadel 2016) and one from Cameroon (Orock et al. 2012) formed four separate, partly supported clades. Unfortunately, the accession from Cameroon was too short to be reliably assessed, missing the entire ITS1 portion (File S1). The ITS identity matrix indicated the largest clade from São Tomé and Príncipe to represent a different species, whereas the two smaller clades closer to $U$. articulata s.str. can be best interpreted as infraspecific lineages (Fig. 17). According to Nadel (2016), specimens in the large, separate clade differed from $U$. articulata s.str. in the pseudocyphellate cortex and the barbatic acid chemistry (protocetraric and fumarprotocetraric acid in U. articulata s.str.; Swinscow \& Krog 1976a; Randlane et al. 2009) and may correspond to U. speciosa Motyka described from São Tomé and

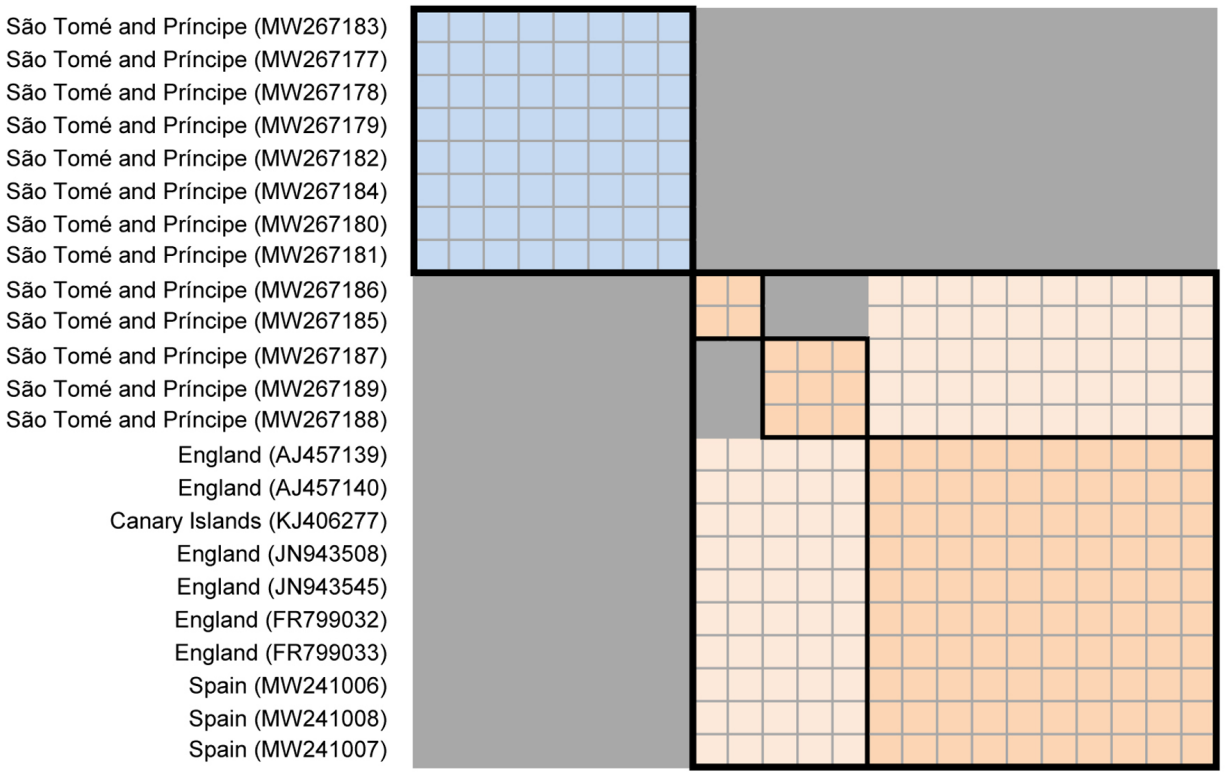

Figure 17. Pairwise identity matrix based on the ITS for the Usnea articulata aggregate after removing four low quality accessions and the short sequence from Cameroon. Dark grey = identity below $98.5 \%$; the blue vs. beige areas indicate separate species, whereas the differently shaded beige areas indicate infraspecific lineages. 


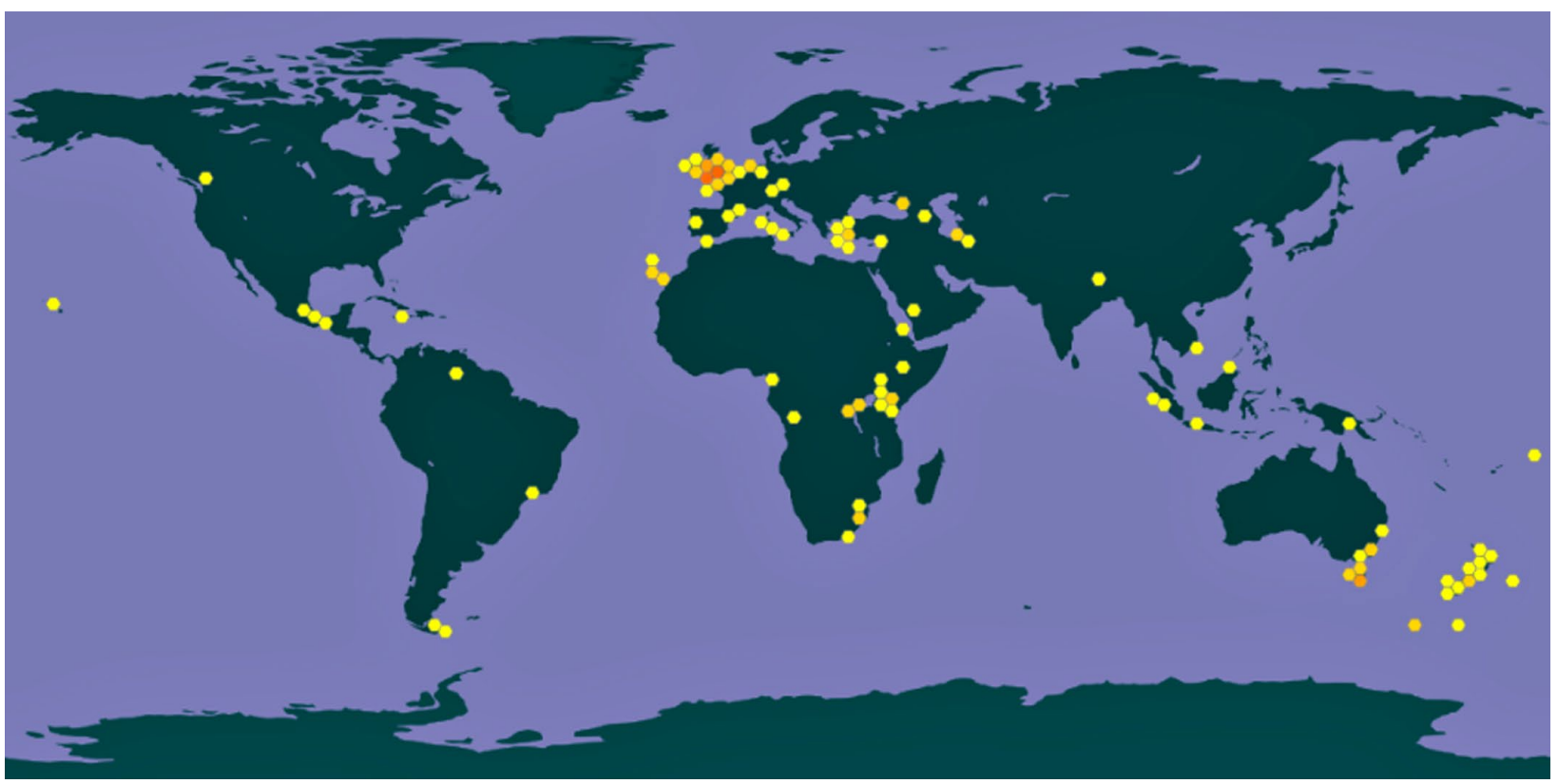

Figure 18. World distribution of Usnea articulata according to the Global Biodiversity Information Facility, GBIF [https://www.gbif.org/species/2606030]; based on available sequence data, many of the extra-European records may not represent that species.

Príncipe (Motyka 1936). This clade somehow intergrades with the unnamed Usnea sp. A, although the latter differs considerably in morphology and chemistry (Nadel. 2016).

The two smaller clades closer to U. articulata s.str. lacked pseudocyphellae and differed in medullary chemistry, both containing psoromic acid (Nadel 2016). According to Swinscow \& Krog (1976a), African material of $U$. articulata represents five chemotypes, whereas only one is known from Europe (Randlane et al. 2009). Given the topology, this supports the notion that African $U$. articulata represents various distinct species, mainly characterized by secondary chemistry and the frequency and nature of the pseudocyphellae. Swinscow \& Krog (1976a) listed several names with variable chemistry as synonyms of $U$. articulata, but did not include $U$. speciosa among them.

Three other markers had more than one sequence for Usnea articulata, namely nuLSU, TUB2, and RPB1, all corresponding to the European core clade. In all three instances, $U$. articulata s.str. was resolved as supported, monophyletic clade, thus congruent with the ITS data, but with better resolution (Figs S2, S4-5).

It must be noted that, while the available sequence data originated from Europe and Africa, Usnea articulata is regarded as a cosmopolitan species (Fig. 18; Swinscow \& Krog 1976a, 1978, 1988; Elix \& McCarthy 1998, 2008; Stevens 1999; Clerc 2007, 2011; Galloway 2007; Randlane et al. 2009; Schumm \& Aptroot 2010; Gumboski \& Eliasaro 2011; Kelly et al. 2011; Saag et al. 2011; Ohmura 2012; Orock et al. 2012; Schoch et al. 2012; Truong et al. 2013a, b; Millanes et al. 2014; Rafat et al. 2015; Nadel 2016; Galinato et al. 2017; Lücking et al. 2020c). The sequenced African samples already indicated that $U$. articulata s.lat. represents a complex of several species. A geographically broader sample is therefore necessary to assess its status, particularly since the species has also been reported from New Zealand (Galloway 2007), but the only available sequence from that region identified with that name was not even related to the European core clade or any of the African lineages.

Usnea barbata-intermedia-Iapponica-substerilis aggregate. A large, unsupported cluster comprising 59 accessions from North America and Europe contained several identifications in largely unresolved patterns (Fig. 7, Table S1, Fig. S1), including U. arizonica (1), U. barbata (14), U. chaetophora (2), U. dasopoga (3), U. diplotypus (3), U. cf. glabrescens (1), U. intermedia (11), U. lapponica (17), U. rigida (1), and U. substerilis (5). Most of these are from the studies by Saag et al. (2011) and Mark et al. (2016a). Usnea arizonica, corresponding to an apparently unpublished sequence (AF297732), was established as a synonym of $U$. intermedia by Clerc (2007). The use of the name $U$. rigida [as $U$. rigida (Ach.) Motyka], corresponding to a sequence (AJ457152) generated by Articus et al. (2002), was clarified by Lendemer \& Tavares (2003a), who established that Motyka (1936) technically described a new species to be cited as U. rigida Motyka, thus creating an illegitimate later homonym of $U$. rigida Vain., a species of Eumitria for which Motyka (1936) had established the replacement name $U$. welwitschiana Motyka. For this reason, Lendemer \& Tavares (2003a) introduced U. quasirigida Lendemer \& I. I. Tav. as the replacement name for Motyka's U. rigida. The latter was listed as a synonym of $U$. intermedia by Clerc (2007), who did not mention the name $U$. quasirigida, but by extension both $U$. rigida sensu Motyka and U. quasirigida are to be considered synonyms of $U$. intermedia. Usnea cf. glabrescens (KU352668; Mark et al. 2016a) was apparently a misidentification, as this taxon clusters in another clade.

This left seven names as identifications in this assemblage. All of these share a similar chemistry with salazinic acid as the main compound and often with protocetraric 
as an accessory compound, with a pendent to subpendent (barbata, chaetophora, dasopoga, intermedia) or subpendent to shrubby (diplotypus, lapponica, substerilis) habit and with soredia and/or isidiomorphs except $U$. intermedia, which produces apothecia (Halonen et al. 1998; Clerc 2007, 2011b; Randlane et al. 2009). Given that $U$. dasopoga formed the bulk of accessions in the next clade (see below), we consider the three identifications in this clade as incorrect (see also Clerc \& Naciri 2021). Two of them clustered with three specimens identified as $U$. diplotypus, one as $U$. barbata, one as $U$. chaetophora, and one unidentified, in a strongly supported subclade. This subclade may be considered to represent $U$. diplotypus s.str., a species characterized by relatively long isidiomorphs (Halonen et al. 1998; Randlane et al. 2009). However, five of the eight samples in this clade were placed with strong support in the $U$. dasopoga core clade in the RPB1 tree, thus providing an unresolved, supported conflict (see below).

The remaining accessions formed five clusters: one supported subclade $(83 \%)$ consisting of four specimens labeled $U$. barbata and $U$. intermedia, one singleton clade on a long branch representing $U$. arizonica, one supported subclade (72\%) including $U$. barbata and $U$. intermedia, one unsupported, homogeneous grade including $U$. barbata, U. chaetophora, U. intermedia (with one low quality accession: JN009731), U. lapponica, and U. substerilis, and one unsupported clade including $U$. lapponica and $U$. substerilis. This topology suggested that $U$. arizonica may indeed be a good species, although more material needs to be studied and sequenced. The remaining accessions formed three groups that may be interpreted as separate species, but the correct nomenclature remains unclear. The first two clades consisted of mixed assemblies of the pendent taxa $U$. barbata (vegetative) and $U$. intermedia (fertile), suggesting that the mode of reproduction may not be a diagnostic feature in this complex, but that indeed two otherwise characterized, separate species are present. This situation is similar to what has been found in the 'species pair' Letharia columbiana vs. L. vulpina (Kroken \& Taylor 2001) or in the case of U. florida vs. U. subfloridana (see below). The remaining accessions seemed to indicate the separation of a shrubby taxon centered around $U$. lapponica, so the morphology of accessions identified as $U$. barbata and $U$. intermedia in this assemblage needs to be revised. However, there was no evidence for a separation of $U$. substerilis from $U$. lapponica. The two taxa are indeed difficult to distinguish, largely based on the shape of the soralia (Halonen et al. 1998; Randlane et al. 2009; Clerc 2011b). The status of U. chaetophora also remains doubtful. The species is distinguished from $U$. dasopoga mainly by the formation of annular cracks (Halonen et al. 1998; Randlane et al. 2009), but the accessions identified with that name fell into three different positions in the tree. Usnea chaetophora has also been considered a morphological variation of $U$. barbata or $U$. dasopoga and has been synonymized with the latter (Clerc 2011b, 2016).

The ITS identity matrix separated Usnea diplotypus from the $U$. barbata-lapponica aggregate, but did not provide clear resolution at the species level for $U$. barbata-intermedia vs. U. lapponica-substerilis (Fig. 19). IGS data for this clade were largely congruent with ITS (Fig. S3). RPB1 did not resolve the barbata-intermedia and lapponica-substerilis subgroups, but place one sample in an entirely different, supported clade with $U$. florida/U. subfloridana (Fig. S5), an indication of a possible paralog.

The complex situation in this group was also discussed by Mark et al. (2016a), who, based on a six-marker analysis found that this entire assemblage, compared to the dasopoga aggregate (see below), exhibited a thinner cortex and a thicker, lax medulla on average. These authors suggested that some of the names established in this complex may merely represent morphological variants, but also found a separation of the pendent $U$. barbata and $U$. intermedia from the subpendent to shrubby $U$. lapponica and $U$. substerilis, proposing synonymy of U. substerilis under $U$. lapponica. We tend to agree with the latter, although the example of $U$. antarctica vs. U. aurantiacoatra (see below) shows that ITS and even multiple markers may not resolve very closely related, recently evolved lineages, which could then also apply to the two clades formed by mixed samples identified as $U$. barbata and U. intermedia, i.e., each clade potentially representing two species. The importance of correct phenotypical assessments of sequenced material has been shown for this group by Clerc \& Naciri (2021). Several accessions of fertile material identified as U. intermedia clustered with $U$. lapponica, suggesting that the latter may have an apotheciate counterpart.

Usnea bismolliuscula-intumescens aggregate. Usnea bismolliuscula is a sorediate species characterized by a shrubby to subpendent habit with small perforations on the branch surface and predominantly producing compounds of the stictic acid chemosyndrome (Ohmura 2001). Usnea intumescens agrees in gross morphology, but lacks perforations and produces salazinic or psoromic acid (Ohmura 2001). It was represented by a singleton sequence (Ohmura 2002).

The four sequences of $U$. bismolliuscula have apparently not been published and so no precise voucher information was available. The singleton sequence was deposited by J.-S. Hur in 2010 and so is possibly from South Korea, and the other three sequences, forming a strongly supported clade on a long branch, originated from Taiwan, deposited by Y.-M. Shen et al. in 2008. It was not possible to determine which of the two lineages, if any, corresponded to U. bismolliuscula s.str. Usnea bismolliuscula was originally described from Japan, as U. molliuscula Vain., an illegitimate later homonym of U. molliuscula Stirt. (Ohmura 2001, 2012). Thus, it is more likely that the singleton presumably from South Korea represents this taxon, whereas the Taiwanese clade would be a different species. Ohmura (2002) distinguished two chemotypes, one with the stictic acid complex that was shown to be widespread in Australasia including Japan and Taiwan, and one with thamnolic acid, only found in Japan. Types of all examined synonyms represented the first chemotype, thus offering no potential name for a deviating tropical lineage present in Taiwan. 


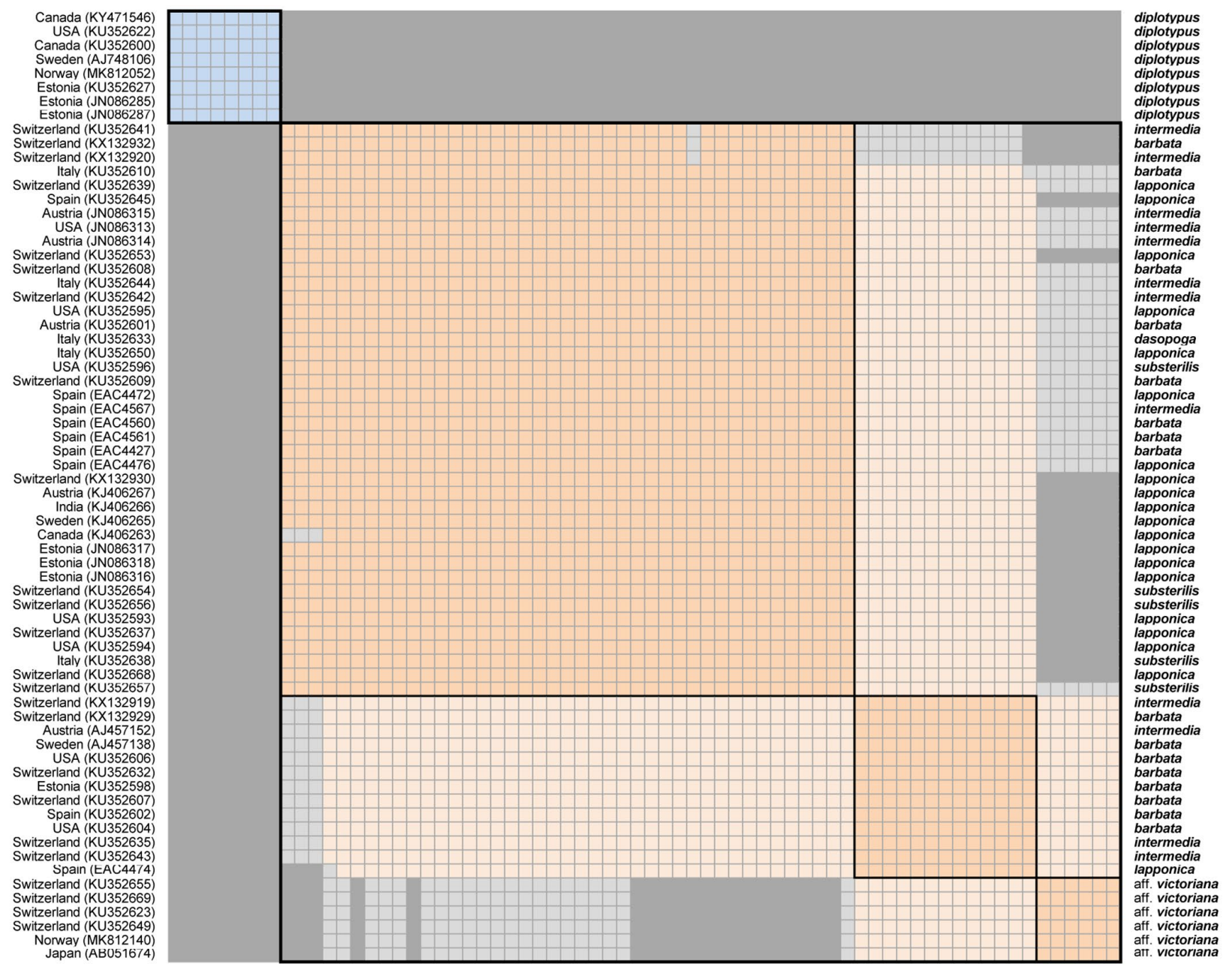

Figure 19. Pairwise identity matrix based on the ITS for the Usnea barbata-intermedia-lapponica-substerilis aggregate after removing four low quality accessions and the short sequence from Cameroon. Dark grey = identity below 98.5\%; light grey = identity exactly $98.5 \%$; the blue vs. beige areas indicate separate species, whereas the differently shaded beige areas indicate insufficiently separated lineages, each with internal identity levels above $98.5 \%$.

Usnea brasiliensis-cornuta aggregate. The Usnea cornuta aggregate exhibited the most complex situation in the genus. No less than 100 accessions bear the names cornuta, aff. cornuta, or subcornuta, distributed in six, partly separate clades containing a total of 208 successions (Fig. 7, Fig. S1). Usnea cornuta s.lat. is defined as a shrubby, sorediate taxon with inflated branches constricted at their point of attachment, minute soralia producing isidiomorphs along the terminal branches, but the taxon was described as highly variable both morphologically and in the medullary chemistry (Clerc 2007; Randlane et al. 2009; Gerlach et al. 2019a, 2020). It has been reported from all major regions and is considered presumably subcosmopolitan (Awasthi 1986; Clerc 1987, 2004, 2006, 2007, 2011b; Marcano et al. 1996; Clerc \& Herrera-Campos 1997; Halonen et al. 1998; Elix \& McCarthy 1998, 2008; Fos \& Clerc 2000; Herrera-Campos et al. 2001; Galloway 2007; Hinds \& Hinds 2007; Randlane et al. 2009; Smith et al. 2009; Kelly et al. 2011; Rodriguez et al. 2011a; Saag et al. 2011; Ohmura 2012; Schoch et al. 2012; Noer et al. 2013; Santiago et al. 2013; Truong et al. 2013a; Millanes et al. 2014; Araujo 2016, as Usnea sp. 1; Chaker 2016; Herrera-Campos 2016; Truong \& Clerc 2016; Ohmura et al. 2017; Rodríguez-R. et al. 2017; Timbreza et al. 2017;
Galinato et al. 2017, 2018; Ohmura \& Kashiwadani 2018; Bungartz et al. 2018; Esslinger 2019; Ohmura \& Clerc 2019; Gerlach et al. 2019a; Lendemer et al. 2019; Lücking et al. 2020c). Truong et al. (2013a) first demonstrated that $U$. cornuta s.lat. was polyphyletic. A much more detailed study of this aggregate, using a multispecies coalescent approach, showed that the $U$. cornuta aggregate (including $U$. brasiliensis and $U$. dasaea) formed 14 different, in part strongly supported lineages with striking correlation with medullary chemistry (Gerlach et al. 2019a). Several of these are newly described in this issue (Gerlach et al. 2020) and there are also several synonyms available to cover potentially distinct lineages (Clerc 2007; Randlane et al. 2009).

The main question is which clade corresponds to Usnea cornuta s.str. The lectotype is from Central Europe (Germany; Clerc 1987, 2004). Within the six clades in the ITS tree containing accessions labeled $U$. cornuta, only three contained subclades with specimens from northern and central Europe. One subclade corresponded to $U$. esperantiana s.str. (see below) and a second one to $U$. flammea. This pointed to a third clade with accessions from Great Britain, France, Spain, Madeira, and Brazil (Kelly et al. 2011; Truong et al. 2013a; Gerlach et al. 
2019a), as representing U. cornuta s.str. (Fig. 7, Fig. S1). This assessment agrees with Gerlach et al. (2019a), who considered $U$. cornuta s.str. to form part of their lineage 5 , and the clade here defined as $U$. cornuta s.str. corresponded to most of their lineage $5 \mathrm{~A}$.

Gerlach et al. (2019a) also combined other small lineages in their lineage 5, largely based on the formation of a single clade with RPBI (Fig. S5), but the ITS data suggest that these may not belong to $U$. cornuta s.str. They were here tentatively labeled $U$. aff. cornuta 1 (Northern Hemisphere), U. aff. cornuta 2 (Peru), $U$. aff. cornuta 3 (Great Britain), U. aff. cornuta 4-7 (all Brazil), and $U$. aff. cornuta 8-9 (USA). A further clade of two unpublished sequences identified as $U$. cornuta from Taiwan, deposited by Y.-M. Shen et al. in 2008, was labeled $U$. aff. cornuta 10 . This clade requires further examination as the two sequences include numerous odd base calls (see above) and $U$. cornuta has not been reported from Taiwan (Ohmura 2001). A similar species described from Japan and also reported from Taiwan, U. confusa, had been synonymized with $U$. cornuta by Clerc (2004). ITS data suggested that $U$. confusa s.lat. formed two distinct clades with unresolved affinities to $U$. cornuta s.str. (Ohmura \& Clerc 2019), although the authors of that study did not arrive at a conclusion of whether $U$. confusa was separate from $U$. cornuta. In our expanded data set, the specimens labeled U. confusa in Ohmura \& Clerc (2019) also formed two clades, clustering in the immediate vicinity of $U$. cornuta s.str. The first clade also included specimens from Brazil and Macaronesia and was relabeled $U$. aff. cornuta 1 , whereas the second was considered to represent $U$. confusa s.str. Overall, the large containing clade of 116 accessions represented the following species and subclades: U. subflammea, U. halei, U. flammea, the newly recognized $U$. tenuicorticata and $U$. trachyclada (Gerlach et al. 2020), U. cornuta s.str., U. aff. cornuta 1-10, U. cirrosa, U. cladocarpa, the newly recognized U. stipitata (Gerlach et al. 2020), and a large, homogeneous clade representing an unidentified species from New Zealand (Fig. 7, Table S1, Fig. S1).

While this complex situation only covered the largest of the six clades, the second largest clade with 44 accessions corresponded mostly to the $U$. esperantiana aggregate (see below). Four of the five accessions deposited under the name $U$. aff. cornuta in this clade represented a newly recognized species, U. kriegeriana ad int. (Gerlach et al., in prep.), which had been labeled sp. 5 or lineage 18 by Gerlach et al. (2019a) and is characterized by a protocetraric acid chemistry. The remaining accession (MF669829) corresponded to lineage 6 in Gerlach et al. (2019a) and was here labeled $U$. aff. kriegeriana.

The third largest clade included 28 accessions representing South America, the Mediterranean, and New Zealand. Named species in this clade included $U$. crocata (South America: Andes), U. grandispora (Brazil), U. macaronesica (Azores), and U. subglabrata (Bolivia). Two newly recognized species were $U$. arianae from South America and Europe and U. rubropallens from Brazil (Gerlach et al. 2020), corresponding to lineages 11 and 13 in Gerlach et al. (2019a). However, based on ITS data,

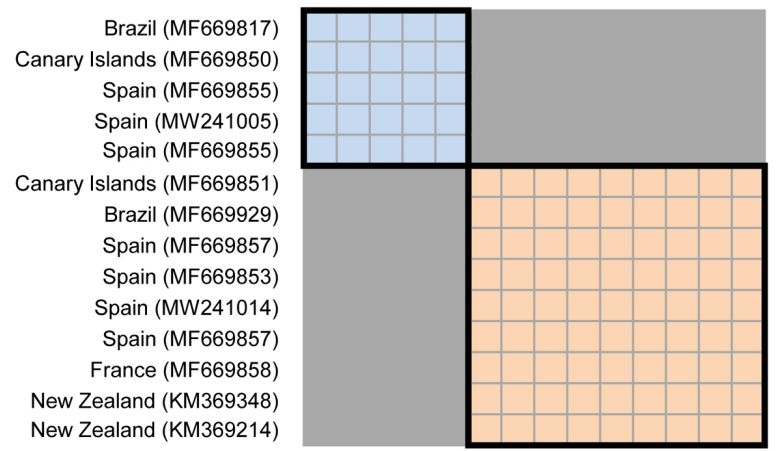

Figure 20. Pairwise identity matrix based on the ITS for the Usnea arianae clade. Dark grey = identity below $98.5 \%$; the blue vs. beige areas indicate separate species, each with internal identity levels above $98.5 \%$.

U. arianae remained heterogeneous (Fig. 7, Fig. S1), as also shown in the ITS identity matrix (Fig. 20), whereas $R P B 1$ resolved these samples in a single clade (Fig. S5). Also, the ITS revealed a subclade of two unidentified sequences from New Zealand (Buckley et al. 2014) nested within $U$. arianae based on the long branch not representing that species in the strict sense. Thus, $U$. arianae appears to be an evolving, subcosmopolitan species complex. A large, unsupported clade with several supported subclades contained accessions labeled $U$. aff. cornuta from South America (Truong et al. 2013a; Gerlach et al. 2019a) and unidentified accessions from New Zealand (Buckley et al. 2014). The South American subclades correspond to lineages 14A-C in Gerlach et al. (2019a) and may represent at least four taxa, here labeled $U$. aff. arianae 1-4.

A singleton sequence from Ecuador (JQ837326) labeled U. subcornuta (Truong et al. 2013a) apparently does not represent that species (see below) and remained unidentified. Two sequences labeled U. cornuta (JN086281, JN086282) from Portugal (Saag et al. 2011) clustered outside the larger clades representing the $U$. cornuta aggregate and instead in the vicinity of $U$. subscabrosa Nyl. ex Motyka. Their identifications also remained unclear. Finally, a clade of 17 accessions from Central and South America, deposited under $U$. cornuta and $U$. aff. cornuta 3, the latter corresponding to lineage 9 in Gerlach et al. (2019a), represent the $U$. subpectinata aggregate (see below).

Usnea brasiliensis was considered a subspecies of U. cornuta by Clerc (2007), but later resurrected based on morphological, chemical and molecular data (Pérez-Vargas et al. 2010a; Truong et al. 2013a; Bungartz et al. 2018; Gerlach et al. 2019a). In the ITS-based phylogeny, eight specimens were labeled $U$. brasiliensis, one from Madeira (Truong et al. 2013a) and seven from Brazil and Costa Rica (Gerlach et al. 2019a). These formed three separate, unrelated clades: one strongly supported clade from Brazil was identified as $U$. brasiliensis s.str., a singleton from Brazil (MF669810) was described as U. flabelliformis, and another, unsupported clade from Costa Rica, Brazil and Madeira was named $U$. tenuicorticata (Gerlach et al. 2020).

No data were available for most of this complex in the IGS, TUB2 and RPB2. RPB1 did provide less resolution 
overall, mostly not discriminating between the lineages affine to Usnea cornuta s.str., but distinct in the ITS (Fig. S5). A similar picture was found for MCM7 that separated the now segregated tropical species (Gerlach et al. 2020) rather well. However, one accession of $U$. tenuicorticata did not cluster with the other two (Fig. S7). In summary, the highly complex Usnea cornuta aggregate corresponds to numerous different species, largely with a strong correlation with medullary chemistry, as already outlined by Gerlach et al. (2019a).

Usnea ceratina aggregate. Usnea ceratina is a (sub-) pendulous, sorediate species characterized by a pink medulla and the production of diffractaic and barbatic acids (Truong \& Clerc 2012). It is presumably cosmopolitan (Marcano et al. 1996; Herrera-Campos et al. 1998; Elix \& McCarthy 1998, 2008; Ohmura 2001, 2002, 2012; Articus et al. 2002; Calvelo \& Liberatore 2002; Clerc 2007; Randlane et al. 2009; Kelly et al. 2011; Truong \& Clerc 2012; Millanes et al. 2014; Herrera-Campos 2016; Sipman \& Aguirre-C. 2016; Mark et al. 2016a; Rodríguez-Flakus et al. 2016; Galinato et al. 2017; Esslinger 2019; Gerlach et al. 2019a; Lücking et al. 2020c).

Our ITS-based phylogeny divided Usnea ceratina into four clades, three smaller clades in a supported, monophyletic group and one large, separate clade (Fig. 7, Table S1, Fig. S1). The large clade, comprised of 14 specimens from Central Europe, Great Britain, and North America, was clearly distinct from the three smaller clades (Fig. 21). It had nine fully consistent and several partial substitutions compared to the smaller clades (File S1). Given that the lectotype of $U$. ceratina is from Poland (Ohmura 2001; Truong \& Clerc 2012), this clade was here interpreted as $U$. ceratina s.str. This clade also included a fertile specimen from Spain identified as U. cristatula (Araujo 2016), which is considered the apotheciate counterpart of U. ceratina (Clerc 2011a; Truong \& Clerc 2012; Gerlach et al. 2017). ITS did not resolve these as separate lineages, a similar case as for $U$. florida versus $U$. subfloridana (see below) and $U$. antarctica versus $U$. aurantiacoatra (see above), but in the latter case, studies with other markers showed that both morphs are indeed phylogenetically different (Grewe et al. 2018; Lagostina et al. 2018). Therefore, caution should be applied before synonymizing taxa based on lack of resolution in the ITS.

The other three clades with specimens labeled Usnea ceratina formed two strongly supported clades from Japan and Taiwan and one unsupported clade from North America, Brazil, and Portugal. These clades were not clearly separated in our identity matrix and could conservatively be considered a single species, although up to three species could be distinguished if a more progressive concept was applied. The two Asian clades are well separated from each other in their identity values, but both overlap with the American-Mediterranean clade (Fig. 21). In any case, the members of this clade are not conspecific with $U$. ceratina s.str. and cannot be identified with that name. For the American-Mediterranean clade, three names are potentially available, U. calicornica Herre, U. subcomosa Vain., U. pachyclada Motyka, and U. solida Motyka (Herrera-Campos et al. 1998; Halonen et al. 1999; Clerc 2007; Truong \& Clerc 2012), U. california having priority. Ohmura (2001) listed two species-level synonyms from Japan under $U$. ceratina, namely $U$. roseola Vain. and U. subroseola Asahina, which would then be available for one or both of the Asian clades. Here, we tentatively used the name roseola (Table S1). Notably, the chemistry of most Asian samples of $U$. ceratina appears to be different, in addition to diffractaic and barbatic acids also including baeomycesic and squamatic acid, which would lend support for the division of the smaller clade into three entities. The name $U$. roseola is actually in use, having been applied to material from North America (Esslinger 2019), Africa (Swinscow \& Krog 1979, 1988), and Australasia (Stevens 1999; Singh \& Sinha 2010). However, it has not been associated with any sequenced specimen so far and hence its use must be revised. With the data at hand, the situation cannot be fully resolved, but for the American-Mediterranean clade, one could adopt the name $U$. californica.

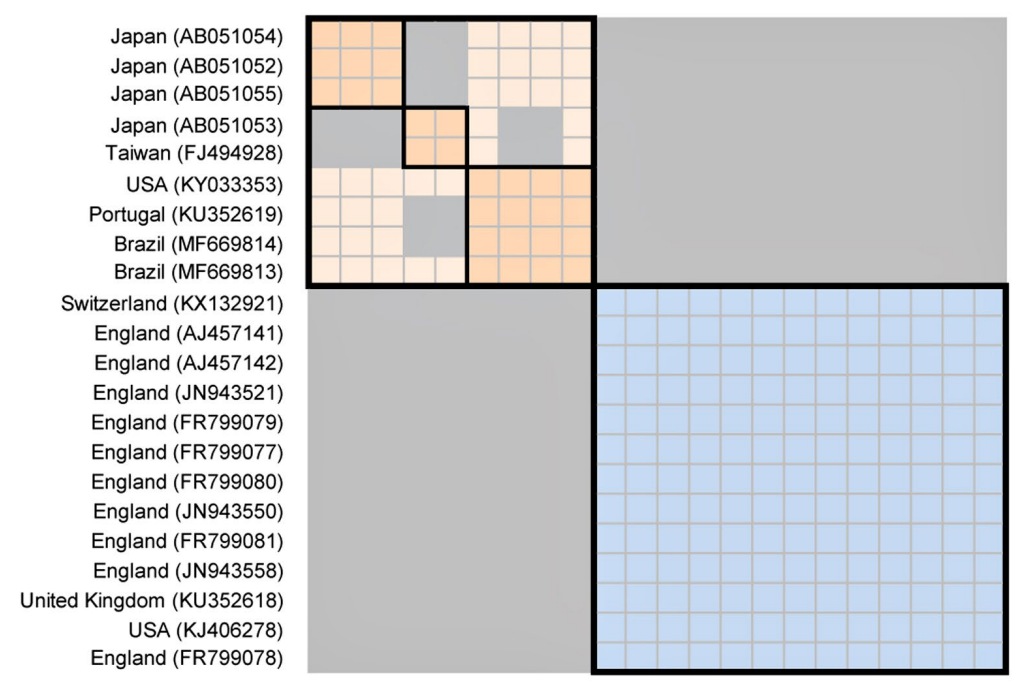

Figure 21. Pairwise identity matrix based on the ITS for the Usnea ceratina aggregate. Dark grey = identity below $98.5 \%$; the blue vs. beige areas indicate separate species, whereas the differently shaded beige areas indicate insufficiently separated lineages. 
There is also a rare chemotype with barbatic acid as only major compound, reported from both the Americas and Asia (Ohmura 2001; Truong \& Clerc 2012). In Asia, the name $U$. roseola subsp. pseudoroseola Asahina corresponds to this chemotype (Ohmura 2001).

Usnea dasaea aggregate. Usnea dasaea is a shrubby to subpendent species with branches constricted at their insertion and with numerous fibrils, producing soralia with isidiomorphs, and with galbinic, norstictic and salazinic acids as major substances. It presumably has a cosmopolitan distribution (Clerc 2007; Randlane et al. 2009) and a large number of listed synonyms, including $U$. dolosa Motyka, U. galbinifera Asahina, U. kinkiensis Asahina, U. kyotoensis Asahina, U. spinigera Asahina, U. spinulifera (Vain.) Motyka and U. undulata Stirt. (Clerc \& Herrera-Campos 1997; Ohmura 2001).

A total of 13 ITS accessions were deposited under this name in GenBank. Given that $U$. dasaea was described from Portugal (Stirton 1881), the clade of two accessions from that country (JN086283, JN086284; Saag et al. 2011) likely represents that species. The remaining 11 accessions represented several other taxa, such as U. grandisora (MF669881), U. aff. perhispidella (MF669883), $U$. subdasaea (four accessions), and U. subpectinata (MF669884), all from Brazil (Gerlach et al. 2019a). Another Brazilian accession (MF669878; Gerlach et al. 2019a) might represent genuine $U$. spinulifera, whereas an accession from Ecuador (JQ837306; Truong et al. 2013a) belongs to an as of yet undescribed species labeled Usnea sp. 6 in Gerlach et al. (2019a). Two further accessions from Japan (AB051056; Ohmura 2002) and Peru (JQ837305; Truong et al. 2013a) represent unnamed lineages.

Usnea dasopoga-viktoriana aggregate. This aggregate formed an unsupported clade following the U. barbata-intermedia-lapponica aggregate (see above). It was also found associated with the latter in the six-marker analysis by Mark et al. (2016a), who described the two clusters to differ in the thickness of the cortex and the extension and consistency of the medulla, while largely agreeing in chemistry. The aggregate contained 43 samples labeled with the following identifications: U. barbata (7), U. cf. cylindrica (1), U. chaetophora (2), U. dasopoga (5), U. diplotypus (2), U. filipendula (17), U. lapponica (2), U. silesiaca (1), U. substerilis (3), U. viktoriana (as U. praetervisa; 3), U. wasmuthii (2), and Usnea sp. (1). Usnea filipendula has been established as a synonym of $U$. dasopoga and the latter as the correct spelling of what has often been named U. dasypoga (Arcadia 2013). Thus, the bulk of identifications in this aggregate corresponded to U. dasopoga (22).

Overall, this aggregate was subdivided into several clusters. A singleton and a doubleton clade were located at the base, including the two likely erroneous identifications as $U$. wasmuthii, close to an unsupported clade containing a strongly supported subclade of three sequences originally identified as U. praetervisa (Mark et al. 2016a), but correctly named U. viktoriana (Clerc \& Otte 2018). The latter is characterized by alectorialic acid, although a smaller number of specimens (not sequenced) produce salazinic and barbatic acids (Clerc \& Otte 2018). Usnea wasmuthii is characterized by salazinic and barbatic acids as major compounds (Ohmura 2001; Randlane et al. 2009; Clerc \& Otte 2018). This taxon clustered in a subsequent clade (Fig. 7, Fig. S1) and hence the two identifications in the $U$. dasopoga aggregate apparently do not represent that species. The small clade including these accessions was here considered as $U$. aff. viktoriana, as the sequences in this portion all share a characteristic ITS sequence motif at the end of the ITS2 (File S1). The singleton sequence labeled $U$. silesiaca in this aggregate from Ecuador (JQ837331; Truong et al. 2013a) contained odd base calls and its correct identification is uncertain. U. silesiaca s.str. clustered in another part of the tree.

The remaining accessions formed a supported clade (74\%) with three clusters: a basal grade of four accessions identified as $U$. barbata and $U$. cf. cylindrica, a strongly supported subclade of six accessions identified as $U$. barbata, U. dasopoga, and U. substerilis, and a remaining shallow grade including the bulk of $U$. dasopoga identifications (Fig. 7, Fig. S1). The entire clade originated from the Northern Hemisphere, mostly from Europe (Table S1). Usnea cylindrica was only recently described from Sweden (Clerc 2011b), differing in branching pattern from $U$. dasopoga, and the only available sequence tentatively identified with that name by Mark et al. (2016a) is probably not that species, a suspicion confirmed by Clerc \& Naciri (2021). Usnea barbata may be similar to $U$. dasopoga and best separated by the thickness of the cortex and the extension and consistency of the medulla (Randlane et al. 2009; Mark et al. 2016a), and so the identifications as $U$. barbata in this clade refer to U. dasopoga (see also Clerc \& Naciri 2021). As a consequence, we consider this clade to represent $U$. dasopoga s.str., with the nested supported subclade requiring further study. Apotheciate specimens of $U$. dasopoga were reported by Lukáč (2010), and so the samples identified as $U$. barbata in this clade were presumed to represent fertile $U$. dasopoga.

The Usnea dasopoga core clade was well supported in both the nuLSU (few data) and the RPBI (Fig. S5). However, the ITS subclade was not obvious from $R P B 1$ data and all specimens were relatively uniform, a pattern also seen with IGS (Fig. S3). Notably, the dasopoga clade in $R P B 1$ contained at least two samples (BAR-08, CHE-09) which based on ITS clustered in the strongly supported diplotypus clade in the barbata-intermedia-lapponica aggregate (see above). This supported conflict needs to be examined further. Mark et al. (2016a) detected similar conflicts, but did not further elaborate on these.

Usnea endochrysea-mutabilis-strigosa aggregate. This aggregate was strongly supported on a long branch in our ITS-based phylogeny (Fig. 7, Fig. S1). Few data were available from other markers, with $U$. endochrysea and $U$. mutabilis forming a monophyletic clade with MCM7 (Fig. S7) and U. mutabilis a monophyletic clade in TUB2 (Fig. S4). With nuLSU, this clade was not recovered as monophyletic (Fig. S2). 
This clade contains species usually having a (pink-) red-pigmented medulla (pigment identified as eumitrin $\mathrm{A}_{2}$ in U. mutabilis) and norstictic acid as the major compound, although $U$. strigosa s.lat. has also been reported as having a white medulla and producing diffractaic, psoromic or thamnolic acid, and U. mutabilis as lacking norstictic acid and producing fatty acids of the murolic acid complex (Hale 1962; Ohmura 2001; Clerc 2007; Randlane et al. 2009; Lendemer et al. 2016). Usnea strigosa and $U$. endochrysea produce apothecia and differ chiefly in ascospore size (smaller vs. larger than $9 \mu \mathrm{m}$ ), although there are conflicting reports in the literature (Clerc 2007; Lendemer et al. 2016). Usnea mutabilis is sorediate. All three species were originally described from North America (Acharius 1803; Stirton 1881), but U. strigosa is considered widespread in the Americas and U. mutabilis subcosmopolitan (Clerc \& Herrera-Campos 1997; LaGreca 1999; Fos \& Clerc 2000; Vareschi 2001; Ohmura 2001, 2002; Calvelo \& Liberatore 2002; Clerc 2007; Mercado-Díaz 2009; Randlane et al. 2009; Saag et al. 2011; Schmull et al. 2011; McDonald et al. 2013; Herrera-Campos 2016; Ohmura \& Kashiwadani 2018; Funk et al. 2018; Esslinger 2019; Dorey et al. 2019; Lendemer et al. 2019; Lücking et al. 2020c).

ITS data did not resolve the three taxa as reciprocally monophyletic and the entire clade exhibited substantial structure. Thus, the six specimens identified as U. strigosa appeared on four clades, two clades clustering on a supported, early diverging branch, one clade clustering with specimens identified as $U$. endochrysea, and one specimen nested within the strongly supported $U$. mutabilis clade, suggesting an apotheciate morph of the latter. Usnea mutabilis formed five supported subclades, but based on the identity matrix (Fig. 22), only the two specimens from Japan could be potentially considered a separate taxon. Specimens identified as U. strigosa and U. endochrysea could be interpreted as representing potentially four lineages, two singletons each corresponding to U. strigosa (MH310890) and U. endochrysea (MH310883), one subclade containing two specimens of $U$. strigosa (AF112990, MN038162), and one with U. endochrysea (MH310884) and U. strigosa (HQ650687, MH310884) intermingled. Unfortunately, with the data at hand it is

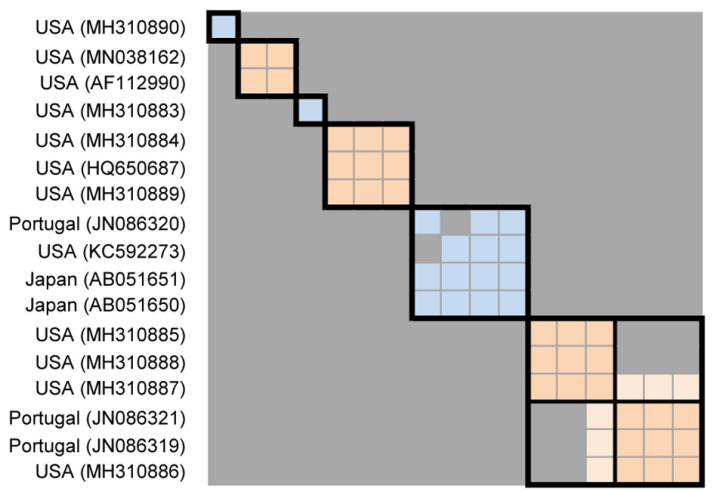

Figure 22. Pairwise identity matrix based on the ITS for the Usnea endochrysea-mutabilis-strigosa aggregate. Dark grey = identity below $98.5 \%$; the blue vs. beige areas indicate separate species, whereas the differently shaded beige areas indicate insufficiently separated lineages. currently not possible to resolve this complex taxonomically or to determine which clade, if at all, represented $U$. strigosa and $U$. endochrysea s.str. Broader sampling and detailed documentation of potentially important characters, such as chemistry and ascospore sizes, is required, as well as reinvestigation of currently listed synonyms.

Usnea erinacea-moreliana-steineri aggregate. This unsupported clade of 19 accessions contained four names that were not resolved in reciprocal monophyly by ITS. All have cortical or subcortical reddish pigments. While $U$. erinacea and $U$. steineri are apotheciate, U. moreliana (including U. rubricornuta) is sorediate. Chemically, terpenoides such as UT6 are common (diagnostic in $U$. moreliana) and U. erinacea also contains protocetraric, norstictic, salazinic and/or stictic acid (Truong et al. 2011; Truong \& Clerc 2016; Gerlach et al. 2017).

Usnea rubricornuta was established by Truong et al. (2011) for a neotropical taxon, but this name was later synonymized with U. moreliana (Truong \& Clerc 2016). Usnea moreliana (as U. rubricornuta) formed a paraphyletic grade relative to $U$. steineri in the analysis by Truong et al. (2013a), but the inclusion of additional samples (Gerlach et al. 2017, 2019a) has rendered the picture more complex. Thus, while U. moreliana s.str. in our tree clustered in a well-supported clade including one accession of $U$. rubricornuta from Bolivia (JQ837323), three other sequences originally identified as $U$. rubricornuta fell outside this clade, one from Brazil clustering with U. erinacea (JQ837322) and another from Peru (JQ837324) with a sequence apparently misidentified as U. rubicunda. It is unclear whether the Bolivian accession actually corresponds to the type of $U$. rubricornuta, as in the various publications, no link was given between the DNA voucher number of the Bolivian accession (34) and the collection number of the type (Truong 3132) and more than one collection from Bolivia was cited in the protologue. The synonymy of $U$. rubricornuta with $U$. moreliana is therefore not definitely established.

Usnea steineri formed three distinct lineages on long branches. Samples identified as $U$. erinacea formed an unsupported clade including one accession of U. rubricornuta. Sequences labeled U. erinacea were also found outside this aggregate, variously clustering with sequences identified as $U$. rubicunda s.lat. (see below), confirming the notion that $U$. erinacea as currently understood is polyphyletic (Truong et al. 2013a; Gerlach et al. 2017).

Another pigmented, apotheciate taxon, Usnea sanguinea, was described from Africa and used to name African and Australian samples corresponding to this morphology (Swinscow \& Krog 1979, 1988; Stevens 1999; Nadel 2016). Clerc (2004, 2007) synonymized $U$. sanguinea with $U$. erinacea. A sequence from São Tomé and Principe labeled $U$. aff. sanguinea (Nadel 2016) fell far from the U. erinacea complex (Fig. 7; Fig. S1), initially supporting the hypothesis that U. sanguinea represents a separate species. This interpretation is, however, tentative, as the single specimen from São Tomé and Principe differs in medullary chemistry from the type of $U$. sanguinea (barbatic vs. norstictic and 
salazinic acid) and was sterile, lacking both apothecia and soredia (Nadel. 2016).

Data from the RPB1 clustered the accessions labeled $U$. rubicunda within $U$. moreliana, which in turn clustered with support with $U$. steineri, whereas $U$. erinacea formed an unsupported clade next to this clade (Fig. S5). Overall, this suggests largely a distinction of three species complexes, U. erinacea, U. moreliana, and U. steineri, but this aggregate requires further study based on a broader sample. Notably, the $M C M 7$ did not resolve these taxa as clusters, but exhibited a confusing pattern suggesting the formation of paralogs (Fig. S7).

Usnea esperantiana aggregate. Usnea esperantiana is a shrubby species forming large soralia and producing salazinic and bourgeanic acids (Clerc 1992, 2007). It appears to be a widespread Northern Hemisphere species reported from North America, Europe, Africa, and Asia (Mies 1989; Clerc 2007; Randlane et al. 2009; Kelly et al. 2011; Saag et al. 2011; Herrera-Campos 2016; Galinato et al. 2017; Esslinger 2019).

In our global phylogeny, sequences deposited under that name formed a monophyletic clade, but with clear internal structure and associated with numerous unidentified sequences from New Zealand (Fig. 7, Table S1, Fig. S1). The entire clade was strongly supported (90\%) and contained five clusters: a fully supported subclade of 11 unidentified specimens from New Zealand (Buckley et al. 2014), a more or less supported clade (70\%) of another seven unidentified specimens from New Zealand from that same study, a further unidentified singleton from
New Zealand, a paraphyletic grade of three specimens, two unidentified from New Zealand and one identified from Great Britain, and a remaining, more or less supported $(72 \%)$ clade of 14 specimens. That larger clade contained mostly samples from Europe identified as U. esperantiana (Kelly et al. 2011; Schoch et al. 2012), but also one sample identified as $U$. aff. glabrata from France (Gerlach et al. 2019a), one unpublished sequence from Taiwan, deposited under the name $U$. glabrata by Y.-M. Shen et al. in 2008, and one unpublished and unidentified sequence from China, deposited by L. Han et al. in 2020 .

The identity matrix (Fig. 23) suggested that the first clade, exclusively from New Zealand, represented a separate species, its identification pending further study based on authentic material. The status of the remaining accessions from New Zealand cannot be assessed. They formed a paraphyletic grade with high similarity to Usnea esperantiana s.str. (Fig. 23), the latter also including two samples from New Zealand, implying that this species also occurs there. Unfortunately, since no voucher material was preserved for these sequences (see above), verification will only be possible with newly collected, properly vouchered and sequenced specimens from that region.

Usnea florida-parafloridana-subfloridana aggregate. Usnea florida and U. subfloridana have been considered a classic example of a 'species pair' (Clerc 1984a) and were the first complex to be studied with molecular data (Articus et al. 2002). Using ITS, nuLSU and TUB2, these authors found that the species pair concept was

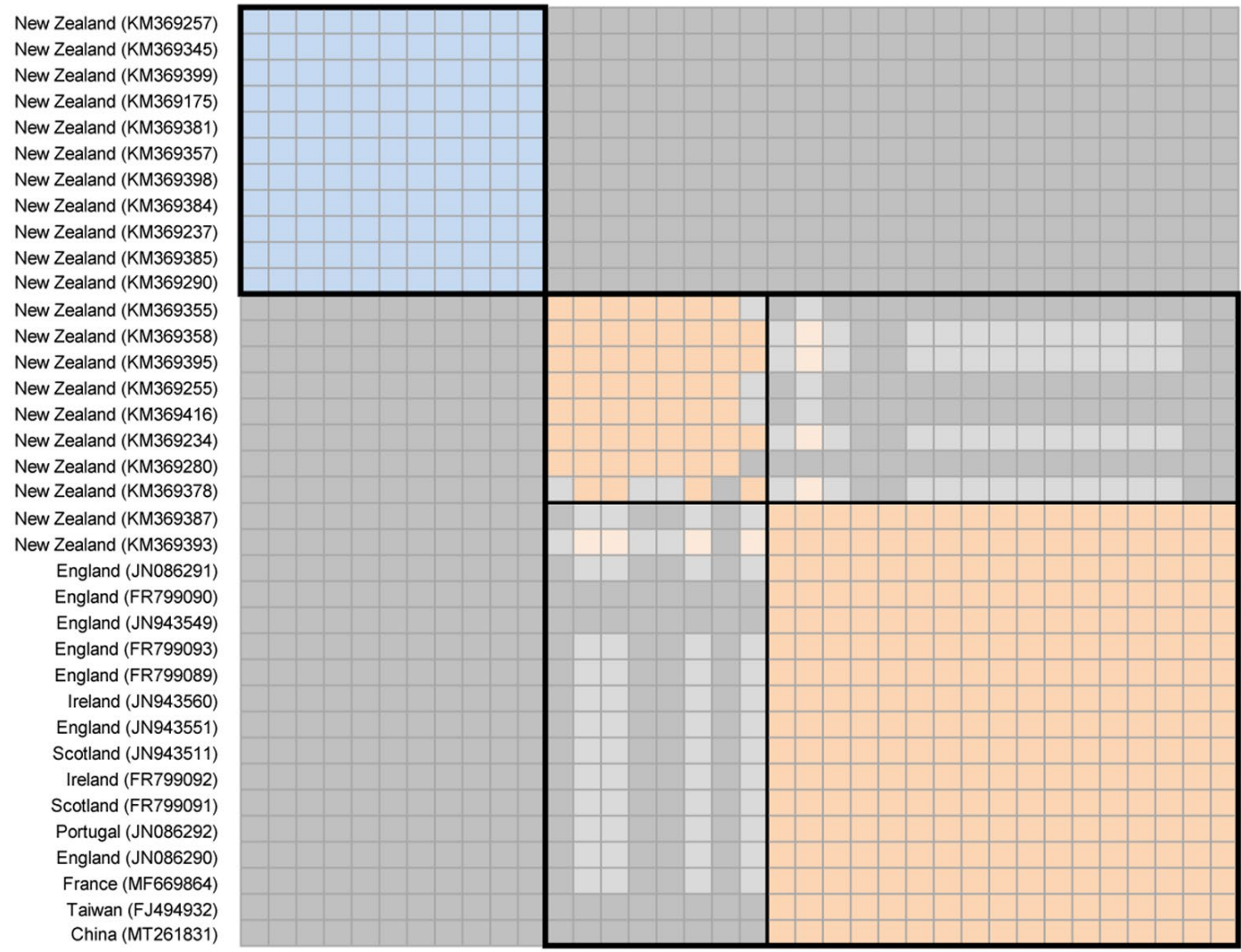

Figure 23. Pairwise identity matrix based on the ITS for the Usnea esperantiana aggregate. Dark grey =identity below $98.5 \%$; the blue vs. beige areas indicate separate species, whereas the differently shaded beige areas indicate insufficiently separated lineages. 


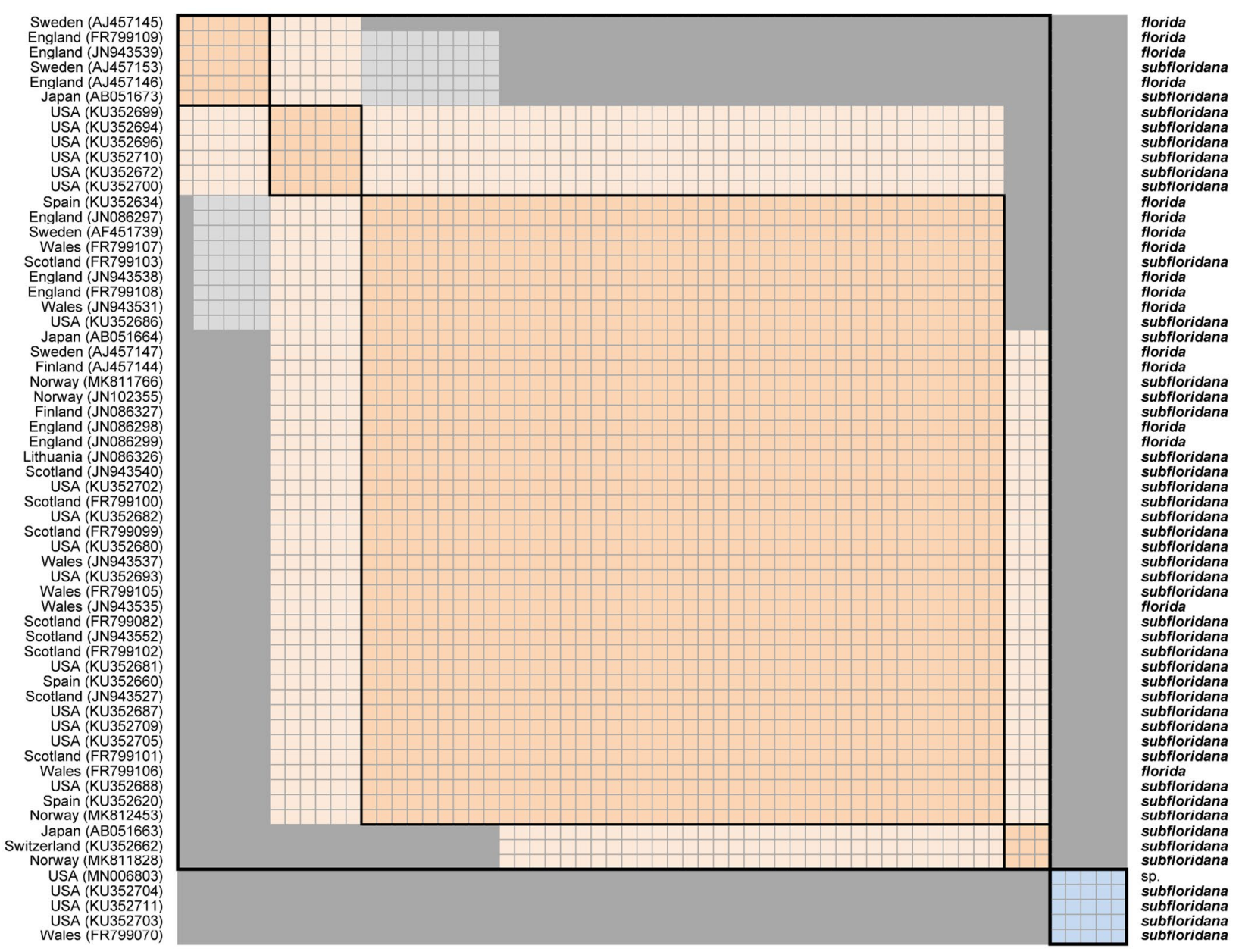

Figure 24. Pairwise identity matrix based on the ITS for the Usnea floridana-subfloridana aggregate. Dark grey = identity below $98.5 \%$; the blue vs. beige areas indicate separate species, whereas the differently shaded beige areas indicate insufficiently separated lineages.

not supported and that instead the studied specimens formed various clades uncorrelated with reproductive mode or chemistry. Both taxa have mostly been reported to contain squamatic and/or thamnolic acid, sometimes in combination with alectorialic acid (Articus et al. 2002; Randlane et al. 2009), but for $U$. florida also norstictic acid has been listed (Clerc 2007). In their six-marker study, Mark et al. (2016a) also found these taxa to form several clades, including one characterized by a norstictic acid chemistry newly described as $U$. parafloridana. The authors thereby overlooked that the name $U$. praetervisa was available for this clade (Clerc \& Otte 2018). Most of the ITS sequences for $U$. praetervisa (as U. parafloridana) formed a homogeneous, weakly supported clade (66\%), with one sequence (KU352670; WW-002) clustering at the base of that clade and another (KU352708; WW-151) clustering elsewhere.

IGS resolved U. praetervisa (as U. parafloridana) as a fully supported clade, with the exception of one accession (KU352588; WW-151), i.e., the same that did not cluster with the others in the ITS (Fig. S3). In contrast, with RPB1, U. praetervisa (as U. parafloridana) formed one grade and one weakly supported clade $(72 \%)$, the latter also including the two outliers (Fig. S5). Thus, while ITS and IGS were largely in line, $R P B 1$ provided a supported conflict and also offered less resolution. MCM7 resolved $U$. praetervisa as to separate, unrelated clades, suggesting a paralog (Fig. S7).

Mark et al. (2016a) suggested that U. florida and $U$. subfloridana may be synonyms, but this was not in accordance with the complex topology in the ITS data, which suggested that several lineages are involved, in part with mixed reproductive strategies, similar to the situation in Letharia colombiana and L. vulpina (Kroken \& Taylor 2001). Indeed, specimens identified as Usnea florida or U. subfloridana clustered in regions in the tree: one supported clade (77\%) containing seven accessions bearing either name (plus an apparently misidentified U. wasmuthii) from across the Northern Hemisphere; one strongly supported clade (99\%) containing five specimens from North America and Europe identified as U. subfloridana; a weakly supported clade (69\%) with seven specimens of $U$. subfloridana from North America; another weakly supported clade (68\%) with 14 accessions bearing either name from North America and Europe; and a large, rather homogeneous assembly of 50 samples also bearing both names from across the Northern Hemisphere (Fig. 7, Fig. S1). The latter included a low-quality sequence (AF117996) on a long branch, explaining the lack of support for this clade. These clades were separated by three other species, namely $U$. wasmuthii, U. fulvoreagens and $U$. glabrescens. The ITS identity matrix only 
supported the consideration of the strongly supported second clade as a potentially distinct species (Fig. 24).

With $R P B 1$, the five clades were not resolved, the corresponding specimens forming a single, supported clade $(75 \%)$ that included a nested, strongly supported (95\%) subclade (Fig. S5). The distribution of specimens in the RPB1 subclades was not correlated with the formation of subclades in the ITS, providing a minor, yet supported conflict between ITS and RPBI when it comes to species delimitation (see above). In this case, $M C M 7$ also resolved the $U$. florida-subfloridana complex in two separate, unrelated clades, suggesting a paralog situation, particularly as in both cases the $U$. praetervisa and $U$. florida/U. subfloridana haplotypes clustered together (Fig. 25; Fig. S7).

It therefore remains unclear how many species are to be distinguished in this complex, what the diagnostic characters would be and whether the reproductive mode played any role in that, and which clade corresponds to the taxa in the strict sense. Particularly, the largest florida-subfloridana clade was reminiscent of the situation in another species pair, Usnea antarctica vs. U. aurantiacoatra, in which microsatellite markers and a RADseq approach demonstrated that the sexual and asexual morphs largely formed separate taxa (Lagostina et al. 2018; Grewe et al. 2018). Tõrra et al. (2014) and Degtjarenko et al. (2018, 2019) developed microsatellite markers for the $U$. subfloridana complex, but did not report on their potential use to separate $U$. florida from $U$. subfloridana.

Usnea fragilescens aggregate. Usnea fragilescens is a shrubby to subpendent species producing large soralia and with a variable chemistry of either stictic and norstictic acids (Europe) or psoromic or salazinic acid (Americas; Clerc 2007; Randlane et al. 2009). Specimens identified with this name mostly formed a homogeneous, strongly supported clade entirely comprised of European accessions (Kelly et al. 2011; Marthinsen et al. 2019), which can be considered $U$. fragilescens s.str. Two specimens from Bolivia, both with salazinic acid (Truong et al. 2013a) clustered at the base of this clade, together forming a supported clade (84\%). Three further sequences identified as $U$. fragilescens (AJ748104, AJ748105: Articus 2004; FR799083: Kelly et al. 2011) clustered in the $U$. cornuta aggregate (see above), a similar species (see Clerc \& Otte 2018 for differences).

For nuLSU, data were available for the Bolivian and European samples (Kelly et al. 2011; Truong et al. 2013a) with a similar topology as with ITS (Fig. S2). With RPB1, the two available sequences of $U$. fragilescens, representing specimens of the European core clade (Kelly et al. 2001) formed a fully supported clade (Fig. S5). No IGS data were available for the species. For $M C M 7$, only the two Bolivian specimens were sequenced (Truong et al. 2013a), also forming a fully supported clade (Fig. S7).

These data suggest that Usnea fragilescens is a phylogenetically well-defined European species, characterized by a stictic acid chemistry, and that the South American salazinic acid lineage forms a closely related, but separate species. Chemical data for the two Canadian specimens
(Articus 2004) were not available, but their phylogenetic placement suggests a more complex situation of deviating chemotypes in relation to the $U$. cornuta aggregate.

Usnea fulvoreagens-glabrescens-pacificana aggregate. Usnea fulvoreagens and U. glabrescens are shrubby, sorediate species forming large soralia and with a variable chemistry, mostly norstictic acid, but usually in variable combinations with other main or accessory compounds, such as stictic, salazinic, protocetraric, diffractaic, or squamatic acids (Clerc 2007; Randlane et al. 2009). The main difference is in the shape of the soralia: ring-shaped, becoming excavate and lacking isidiomorphs in $U$. fulvoreagens vs. punctiform to maculiform, remaining discrete and producing isidiomorphs in U. glabrescens (Randlane et al. 2009). In Far East Russia and Asia, U. fulvoreagens contains also zeorin, whereas $U$. glabrescens does not (Ohmura 2001, 2012; Ohmura et al. 2017). According Halonen et al. (1999), the delimitation of U. fulvoreagens and $U$. glabrescens can be difficult and $U$. fulvoreagens was considered a synonym of $U$. glabrescens by Clerc (2011b). Clerc \& Otte (2018) opted for the recognition of two varieties: var. glabrescens, with three chemotypes, occurring in boreal and montane temperate areas, and var. fulvoreagens Räsänen, also with three chemotypes, with a boreal-montane to sub-Mediterranean distribution.

In the ITS-based phylogeny, the two taxa were not resolved in reciprocal monophyly, but there was also no evidence that they represent a single species. Usnea fulvoreagens formed one well-supported subclade from southern Europe and Japan, one grade from North America, and one larger, strongly supported clade from northern and northwestern Europe and North America. This separation was also reflected in the IGS and RPBI data (Figs S3, S5). We considered the larger subclade to represent $U$. fulvoreagens s.str. and the small subclade and the grade two potentially separate species. Usnea glabrescens formed a small grade including two samples originally identified as $U$. diplotypus and $U$. substerilis (Saag et al. 2011), plus a larger, unsupported clade from across the Northern Hemisphere, including two specimens originally identified as U. pacificana and U. substerilis (Fig. 7; Fig. S1).

Usnea pacificana was rather recently described from North America (Halonen 2000), morphologically similar to $U$. glabrescens, but with a chemistry of baeomycesic and squamatic acid. In the protologue, it was not compared to $U$. glabrescens. The species was subsequently also reported from Turkey (Senkardesler \& Clerc 2017). Mark et al. (2016a) listed two ITS accessions under U. pacificana in their voucher table, one (KU352651) originally deposited under that name and the other (JN086329) originally named U. substerilis (Saag et al. 2011). Only the second was used in the species delimitation analysis by Mark et al. (2016a) and tentatively accepted as representing $U$. pacificana. It came out on a separate, longer branch, but this was likely caused by three aberrant base calls that may represent reading errors, as they appear in sites otherwise constant for all other sequences in this portion of the tree. Apart from these three base calls, the 


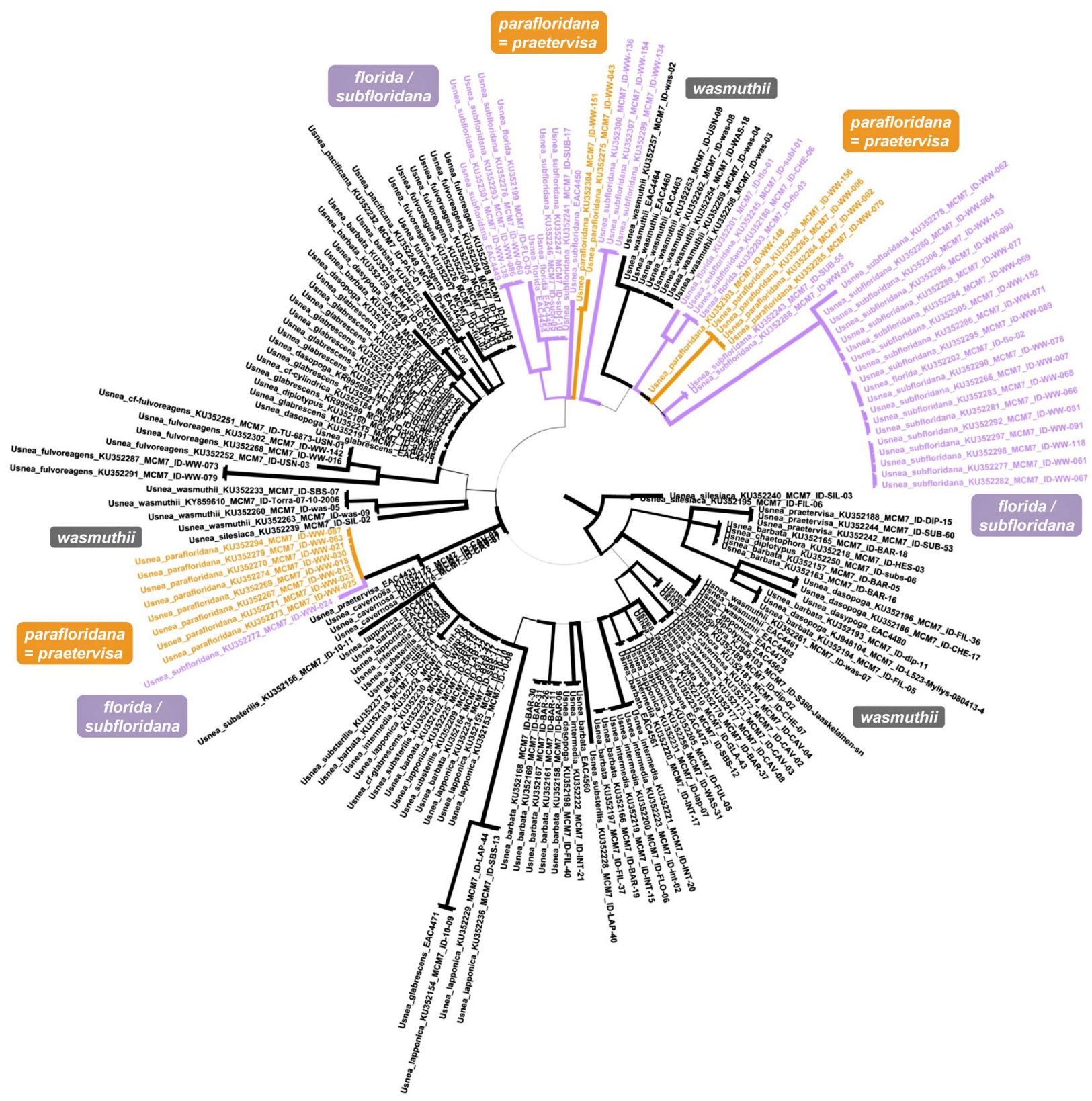

Figure 25. Circle phylogram of Usnea sect. Usnea based on MCM7, highlighting the formation of at least two paralogs in the U. florida-subfloridana complex and U. praetervisa (originally labeled U. parafloridana), also underlined by the relative position of $U$. wasmuthii (see Fig. 16 for comparison). The second cluster on the upper right might reflect an additional, recent duplication event.

ITS was identical to that of two other accessions originally labeled U. pacificana (KU352651; Mark et al. 2016a) and U. glabrescens (AB051639; Ohmura 2002), and the three accessions differed from those of $U$. glabescens s.str. only in one position (File S1). Mark et al. (2016a) gave the chemistry of their $U$. pacificana clade as baeomycesic and squamatic acid, but it is unclear whether this refers to the actual specimens or deduced from the published chemistry for $U$. pacificana. The fact that Saag et al. (2011) identified the corresponding accession (JN086329) originally as $U$. substerilis suggests that it contains norstictic and salazinic acid, and the same applies to the accession labeled U. glabrescens (AB051639) by Ohmura (2002). Mark et al. (2016a) suggested that the baeomycesic and squamatic acid chemotype may occur variably in the U. glabrescens-fulvoreagens complex, which, given the
ITS-based topology, makes the distinction of $U$. pacificana based on medullary chemistry questionable. RPB1 data did not resolve this problem. Although two accessions labeled U. pacificana (KU352077, KU352094) formed a grade basal to $U$. glabrescens, another accession labeled $U$. cf. fulvoreagens (KU352096), presumably with norstictic and salazinic acid, also clustered within that grade (Fig. S5). IGS data, also with two accessions for U. pacificana, did not separate the latter from U. glabrescens (Fig. S3).

Usnea glabrata aggregate. Usnea glabrata is another shrubby, sorediate species, characterized by swollen branches, large and excavate soralia lacking isidiomorphs, a lax medulla, and usually with protocetraric (and fumarprotocetraric) acid, although a salazinic-norstictic 
acid chemotype is also known (Clerc 2007; Randlane et al. 2009). Eight ITS accessions have been deposited under this name (one as $U$. aff. glabrata). Two of them represented $U$. esperantiana (see above), whereas the remaining six clustered with an unidentified sequence in a supported clade (78\%) sister to U. durietzii (Fig. 7, Fig. S1). These seven accessions formed two lineages: a singleton sequence from Switzerland on a rather long branch and a cluster of five sequences from the USA, Spain and Great Britain on a well-supported branch; the seventh sequence clustering at the base also belonged here but was very short (see above). Assuming that these accessions represented two species, it remains unclear which corresponded to U. glabrata s.str., as the latter was described from Switzerland and so both lineages may correspond to this taxon. With nuLSU, the two lineages formed a single, supported and homogeneous cluster. Data available for the $R P B I$ corresponded to the second lineage, forming a fully supported clade on a long branch (Fig. S5).

Usnea himalayana-nidifica aggregate. This small, strongly supported clade $(99 \%)$ contained nine accessions. Two unidentified accessions from New Zealand (Buckley et al. 2014) formed a fully supported subclade on a long branch. A second, strongly supported subclade (99\%) consisted of two unpublished sequences identified as U. himalayana from Taiwan, deposited by Y.-M. Shen et al. in 2008. A third, supported subclade (74\%) contained three lineages: two originally unidentified sequences from Taiwan labeled U. pseudogatae in the corresponding paper (Shen et al. 2012), one sequence from Malaysia labeled U. himalayana (Ohmura 2002), and two unpublished sequences from Taiwan identified as U. nidifica, also deposited by Y.-M. Shen et al. in 2008. Given that $U$. himalayana was described from India (Babington 1852) and the specimens from tropical southeast Asia formed two separate lineages, it is possible that neither of these actually represents that species.

Usnea perhispidella-aciculifera-subpectinata aggregate. Ten sequences bear the name Usnea perhispidella, a species described from Kenya (Steiner 1897). This taxon is characterized by a subpendulous thallus with a pale base and minute, plain, densely arranged soralia combined with numerous isidiomorphs and isidiofibrils, and the stictic acid complex chemistry (Clerc 2016). Nine Brazilian samples identified with this name formed a monophyletic, supported clade in the analysis by Gerlach et al. (2019a), including also $M C M 7$ and $R P B 1$. however, the internal structure suggested the presence of more than one species (Gerlach et al. 2019a: fig. 2).

In our full ITS analysis, several additional specimens clustered with these samples phylogenetically, forming four groups. Three samples from Brazil (MF669898, MF669903, MF669905) and one unpublished sequence of unknown origin, but likely from Southeast Asia (HQ671307), formed a cluster here interpreted as $U$. perhispidella s.str. Closely related to this cluster was a strongly supported clade containing six terminals and differing from the $U$. perhispidella cluster in five consistent substitutions: one from Taiwan (FJ494923), one from Japan (AB051049), two from China (MT259216, MT259217), and two from Brazil (MF669900, MF669899). The four Southeast Asian sequences were deposited under the name Usnea aciculifera Vain. and the Japanese sample was published under this name (Ohmura 2002), whereas the other three sequences have apparently not been published. Clerc (2016) discussed $U$. aciculifera in connection with $U$. perhispidella, maintaining $U$. aciculifera as a different species, with aggregated soralia and short isidiomorphs rarely forming isidiofibrils. Assuming that the identifications of the Asian specimens are correct, which appears likely (Ohmura 2001, 2002, 2012), the ITS data supported the separation of $U$. aciculifera from $U$. perhispidella and also indicate that both species have a wide, possibly pantropical distribution. In that case, the two Brazilian specimens should correspond to the aciculifera and not the perhispidella morphology.

Three further specimens from Brazil (MF669904, MF669879, MF669902) included in the Usnea perhispidella clade by Gerlach et al. (2019a) did not cluster with the above two groups, but from two separate, small clades phylogenetically more similar to two specimens from Peru (JQ837305) and Japan (AB051056) identified as U. dasaea (Ohmura 2002; Truong et al. 2013a), but apparently not representing that species (see above), and a large cluster of unidentified specimens from New Zealand for which no voucher information was available (Buckley et al. 2014). The two small $U$. perhispidella s.lat. clades were here labeled $U$. aff. perhispidella 1 (MF669904) and U. aff. perhispidella 2 (MF669879 / MF669902).

A specimen identified as Usnea perhispidella from Peru (JQ837290; Truong et al. 2013a) clustered with a specimen deposited as $U$. dasaea, but published under the name U. perhispidella from Brazil (MF669883; Gerlach et al. 2019a). This strongly supported small clade was found to be more distantly related to the $U$. perhispidella / U. aciculifera cluster (separated by the U. subpectinata complex, see below) were and is here labeled $U$. aff. perhispidella 3.

The Usnea subpectinata complex formed an unsupported clade of accessions originally deposited under the names $U$. cornuta and $U$. aff. cornuta 3 (Gerlach et al. 2019a; see above). Usnea subpectinata, described from Scotland, was considered a synonym of $U$. cornuta (Clerc 2004, 2006). The clade labeled here with this name contained mostly accessions from Central and South America, but also one from Europe (France). It formed a monophyletic, but unsupported clade in the ITS, including three supported subclades (Fig. 7, Fig. S1). The first of these, here labeled $U$. aff. subpectinata, could be considered a separate species based on the number of consistent substitutions (File S1). The other two subclades, although strongly supported, differed from each other at the $98.5 \%$ threshold, but not from the other samples in this clade. The RPB1 recovered the entire clade (except $U$. aff. subpectinata) as well-supported (87\%) with only little internal structure and uncorrelated with the subclades in the ITS (Fig. S5). With $M C M 7$, two larger, strongly supported, but entirely separate clades, were found, plus 
one smaller, supported clade, a potential indication of the presence of three paralogs (Fig. S7).

Usnea pygmoidea-orientalis aggregate. This unsupported clade contained eight accessions, all from Asia: two from Japan identified as U. pygmoidea (Ohmura 2002), four from Taiwan identified as $U$. orientalis (Shen et al. 2012), and two unidentified and unpublished sequences from China forming a fully supported subclade. Neither $U$. pygmoidea nor $U$. orientalis were resolved as monophyletic. The two taxa form shrubby to subpendent thalli and $U$. orientalis produces apothecia, whereas $U$. pygmoidea forms soralia. U. pygmoidea is considered to include two chemical races, one corresponding to the chemistry of $U$. orientalis in producing salazinic acid as major compound (Ohmura 2001). This suggests a complex situation of a species pair aggregate.

Usnea rubicunda-rubrotincta aggregate. Usnea rubicunda is one of the most commonly collected species of Usnea, with a presumably cosmopolitan distribution (Fig. 26), characterized by a reddish cortical pigment, small soralia producing isidiomorphs, and a stictic acid chemistry (Swinscow \& Krog 1979, 1988; Mies 1989; Marcano et al. 1996; Elix \& McCarthy 1998, 2008; Stevens 1999; Aptroot \& Seaward 1999; Ohmura 2002, 2008; Calvelo \& Liberatore 2002; Spielmann 2006; Galloway 2007; Clerc 2007; Mercado-Díaz 2009; Randlane et al. 2009; Schumm \& Aptroot 2010; Singh \& Sinha 2010; Kelly et al. 2011; Saag et al. 2011; Truong et al. 2011, 2013a; Millanes et al. 2014; Aptroot 2016; Herrera-Campos 2016; Sipman \& Aguirre-C. 2016; Truong \& Clerc 2016; Ohmura \& Kashiwadani 2018; Rodríguez-Flakus et al. 2016; Galinato et al. 2017; Gerlach et al. 2017, 2019a; Bungartz et al. 2018; Esslinger 2019; Lücking et al. 2020c).
A total of 34 ITS sequences have been deposited under the name Usnea rubicunda, originating from North and South America, Europe, and Asia (Ohmura 2002, 2008; Kelly et al. 2011; Saag et al. 2011; Schoch et al. 2012; Truong et al. 2013a; Millanes et al. 2014; Gerlach et al. 2017, 2019a). Most of these clustered in the same part of the tree, but did not form a coherent entity (Fig. 7, Table S1, Fig. S1). One supported clade (83\%) contained 13 samples from North and South America, Macaronesia, the Mediterranean, and Asia, including one sequence from Brazil (MF669912) identified as U. aff. rubicunda (Gerlach et al. 2019a) and one unpublished sequence from the USA deposited by Z. R. Caven et al. in 2016, identified as $U$. pennsylvanica, presumably a synonym of $U$. rubicunda (Clerc 2007). A second, unsupported clade contained 18 sequences, one of them (JQ837307) identified as U. erina$c e a$, apparently a fertile specimen not related to the latter species. A third, internally structured clade was supported $(83 \%)$ and contained mostly unidentified sequences from New Zealand (Buckley et al. 2014), one sequence identified as $U$. rubicunda from New Zealand (Millanes et al. 2014), and three sequences labeled U. rubrotincta from Asia (Ohmura 2002; Ohmura \& Clerc 2019).

The first clade contained at least three supported lineages: one with five terminals including the single U. pennsylvanica sample from North and South America and the Mediterranean; a second lineage with three terminals from Asia, and a third lineage with five terminals from North and South America and Asia. The second clade contained mostly a consistent haplotype, present in South America and Europe, and a few deviating singletons from South America, Galapagos, and Macaronesia. The third clade contained up to four units, all from Asia and/or New Zealand, three of them remaining unnamed and the fourth, a grade with four sequences, bearing the labels $U$. rubicunda and $U$. rubrotincta. Given that the type

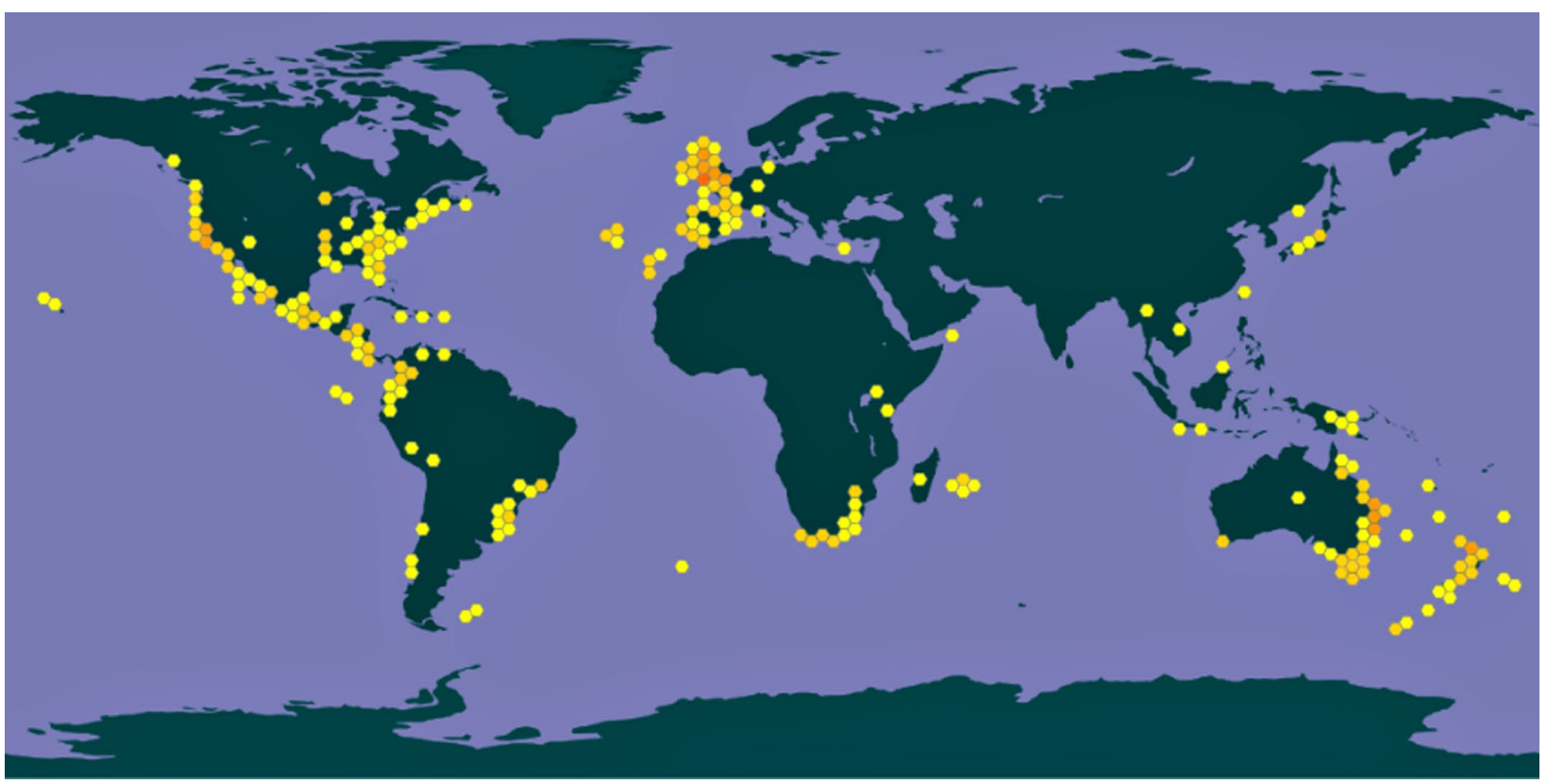

Figure 26. World distribution of Usnea rubicunda s.lat. according to the Global Biodiversity Information Facility, GBIF [https://www.gbif.org/ species/2606096]. 


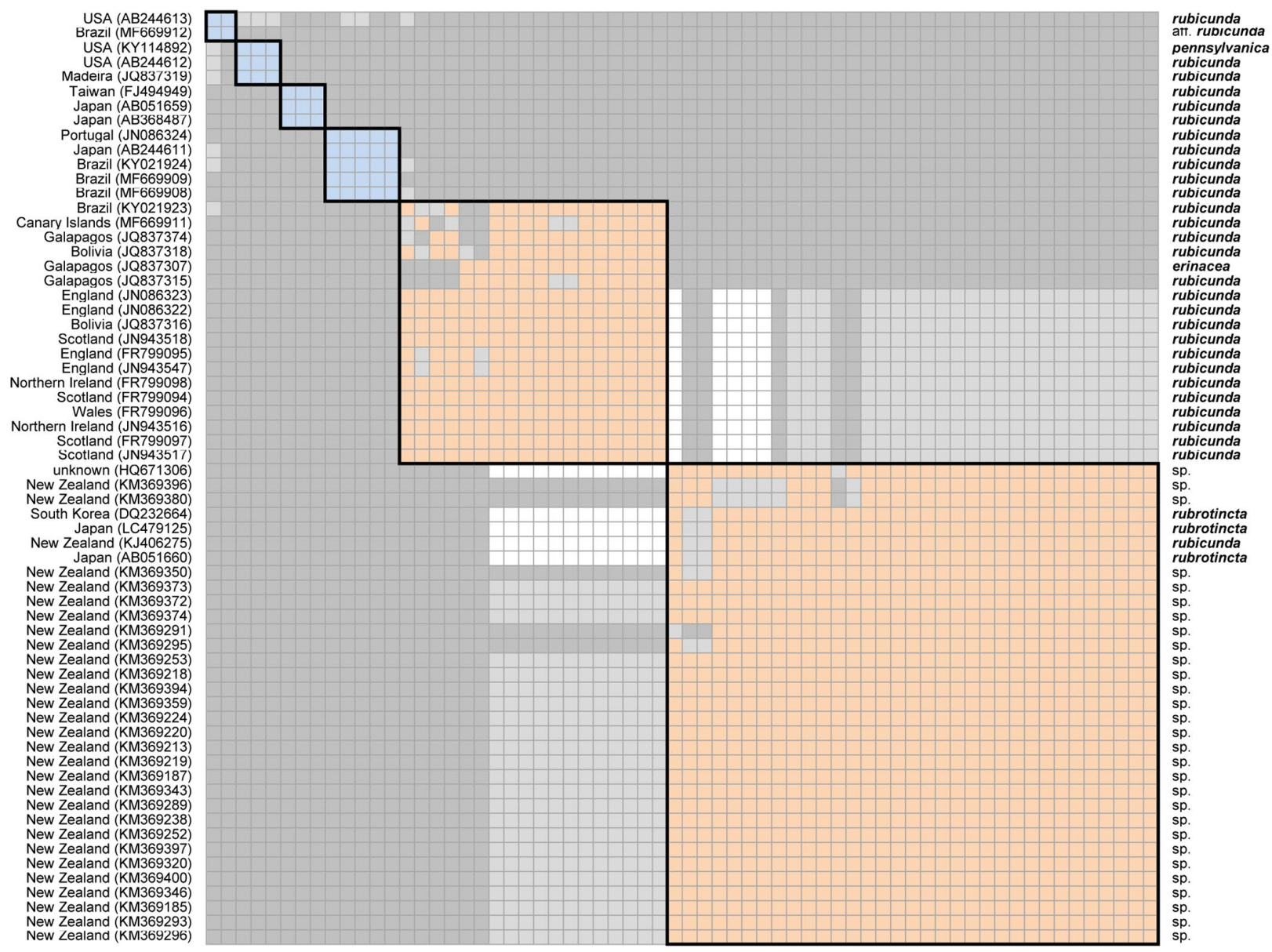

Figure 27. Pairwise identity matrix based on the ITS for the Usnea rubicunda aggregate. Dark grey = identity below $98.5 \%$; the blue vs. beige areas indicate separate species, whereas the differently shaded beige areas indicate insufficiently separated lineages.

of U. rubicunda is from Great Britain (Stirton 1881), we considered the cluster including all specimens from Great Britain and several from South America as U. rubicunda s.str., further encompassing the somewhat deviating haplotypes from Galapagos and parts of South America (Fig. 27). The Asian-New Zealand clade was not well separated from the $U$. rubicunda cluster in the identity matrix (Fig. 27) and may be recognized at the subspecies level.

In contrast, the first clade contained four well separated entities in topology, support and in the identity matrix (Fig. 27). These should be considered separate, likely cryptic species. For the second clade, the name $U$. pennsylvanica appears to be available. For the third clade from Asia, the name U. pseudorubicunda Asahina would potentially apply (Ohmura 2001), whereas for the fourth clade, including specimens from South America, the Mediterranean, and Asia (Japan), the situation is more complicated. This entire assembly requires detailed study, including potential phenotypic differences and the proper assessment of type material. Some additional sequences originally deposited under the name Usnea rubicunda were found in other clades, here labeled $U$. aff. moreliana and $U$. aff. rubrotincta.

Clerc (2007) listed Usnea rubrotincta (described from Madeira) and $U$. rubescens (described from Australia) as synonyms of $U$. rubicunda. Ohmura (2008) performed a detailed morphological, chemical and phylogenetic analysis, resulting in a clear separation of $U$. rubrotincta from $U$. rubicunda. However, all sequenced specimens identified as $U$. rubicunda in that study corresponded to the lineages in the first clade, thus not representing $U$. rubicunda s.str. Usnea rubrotincta itself formed a core clade separate from $U$. rubicunda in another section of the tree (Fig. 7, Table S1, Fig. S1) with three sequences from Japan (Ohmura 2002, 2008) and two unpublished sequences from Taiwan deposited by Y.-M. Shen et al. in 2012. Three further sequences identified as $U$. rubrotincta from Japan (Ohmura 2002; Ohmura \& Clerc 2019) and South Korea (deposited by K.-M. Lim et al. in 2005, but apparently not used in a published study), did not cluster with the other sequences, but fell into the third clade in the U. rubicunda assemblage (see above). Ohmura (2008) recovered all of these in a single clade, likely because of the limited taxon sampling and outgroup selection.

Thus, while there are two clearly separate entities corresponding to Usnea rubrotincta, it remains unclear how the name Usnea rubrotincta is to be applied, given that specimens identified with that name, all from Asia, formed two distantly related clades in different portions of the tree and that the type material is from Madeira.

Usnea subcornuta aggregate. This is another shrubby, sorediate species, characterized by a subcortical, orangered pigment, large soralia with isidiomorphs, and a stictic-norstictic acid chemistry (Randlane et al. 2009; Truong $\&$ Clerc 2016). Four ITS accessions were available under 
this name, forming three lineages in two different places of the tree (Fig. 7, Fig. S1). One sequence from Ecuador (Truong et al. 2013a) fell close to several unidentified accessions from New Zealand, whereas the other three sequences formed two lineages in close proximity, one singleton from Ecuador (Truong et al. 2013a) and two from Europe (Saag et al. 2011, Truong et al. 2013a). Given that U. subcornuta was described from Madeira (Stirton 1881), the European clade of two samples likely corresponds to this species. The related singleton sequence from Ecuador (JQ837327) contained some odd base calls, so its status requires further examination.

Data from the nuLSU also placed Usnea subcornuta sequences as singletons in three separate lineages (Fig. S2), whereas with $M C M 7$, one Ecuadorian and one European sequence clustered in a strongly supported clade, albeit on long individual branches (Fig. S7). The first Ecuadorian sample was found to be unrelated to U. subcornuta s.str., whereas the second sample from Ecuador appeared to represent a distinct, yet closely related lineage.

Usnea subflammea aggregate. Usnea subflammea was recently described from the Azores and the Canary Islands (Clerc 2006), characterized as being similar to U. flammea, but with a (sub-)pendent growth habit, numerous surface tubercles and absence of lobaric acid (stictic acid only). Four accessions have been deposited under this name, two from Brazil and two from the Canary Islands (Gerlach et al. 2019a). In the study by Gerlach et al. (2019a), one of the sequences from the Canary Islands $(238 \mathrm{ES}=238 \mathrm{TEN})$ was labeled $U$. geisleriana, and all four fell in the same area of the species tree together with a sequence identified as U. flammea. The latter was here revealed to form part of U. flammea s.str. (Fig. 7, Fig. S1), whereas the two accessions from the Canary Islands formed two separate lineages in proximity to each other, but not a single clade. The two accessions from Brazil fell in a separate area of the ITS tree, there clustering with weak support (70\%). $R P B 1$ also separated the four accessions into three clades (Fig. S5), similar to $M C M 7$ (Fig. S7), supporting the interpretation of the sample 238ES (=238TEN) as representing $U$. geisleriana and the Brazilian lineage as a different taxon resembling $U$. subflammea phenotypically (U. aff. subflammea).

Usnea subscabrosa aggregate. Usnea subscabrosa was described from Portugal (Motyka 1938). It is a shrubby to (sub-)pendent species characterized mainly by a thick, hard and vitreous cortex, small soralia and by the presence of protocetraric acid in the medulla (Truong et al. 2013b). A second chemotype with thamnolic acid occurs in the Azores (Clerc 2006) and in the Iberian Peninsula (Araujo 2016), but sequences for this chemotype are not yet available.

In our ITS-based tree, the five available sequences labeled U. subscabrosa formed an unsupported clade with two strongly to fully supported subclades (Fig. 7; Fig. S1): one with one accession from North America (apparently unpublished; origin confirmed by consultation of the NYBG C. V. Starr Virtual Herbarium at http:// sweetgum.nybg.org/science/vh) and two from Europe (Spain; Araujo 2016), and a second subclade with two accessions from Brazil (Gerlach et al. 2019a). The first subclade was here interpreted as U. subscabrosa s.str., whereas the Brazilian subclade represents a separate species ( $U$. aff. subscabrosa). The two subclades differed in 12 consistent substitutions and one indel (File S1), resulting in $97.8 \%$ similarity. MCM7 data were available for the two Brazilian samples, forming two separate singletons on long branches (Fig. S7), another example of a potential paralog situation in this marker.

\section{ITS-based DNA barcoding in Usnea: current versus updated metadata}

Comparison of updated identifications of sequence labels with the submitted identifications resulted in seven scenarios: (1) the submitted name matched the name in a phylogenetic species concept (exact match); (2) the submitted name matched an established synonym of the updated name (exact match, but use of a synonym; e.g., Usnea filipendula vs. U. dasopoga; see Arcadia 2013); (3) the submitted identification was unnamed, but had a specific label denoting species-level precision (precise, but unnamed; e.g., Usnea sp. NW-2007-1 in Wirtz et al. 2008 or $U$. aff. cornuta 3 in Gerlach et al. 2019a); (4) the submitted name matched the correct species complex, but did not identify the precise subclade (imprecise; e.g., U. baileyi from Hawaii in Ohmura 2002 vs. U. baileyi from Cameroon in Orock et al. 2012); (5) the submitted name matched the corresponding species phenotypically, but corresponded to a separate, sometimes unrelated clade, not the species in a strict sense (misleading; e.g., U. cornuta from Ecuador and Peru in Truong et al. 2013a); (6) the submitted name did not have a species-level identification, e.g., Usnea sp. (unresolved); and (7) the submitted name identified a species different from the actual species represented by the underlying sequence data (incorrect; e.g., U. malmei from Taiwan in Greenwood et al. 2016).

For DNA barcoding to work well, one would expect most accessions to belong to categories 1-4. However, in our assessment of original submission labels, only about half of the sequences provided an exact match, $47 \%$ corresponding to the current name and $2 \%$ to an established synonym (Fig. 28). Furthermore, 5\% provided a precise, yet unnamed match and $12 \%$ corresponded to species complexes (imprecise). A total of 35\%, i.e., one third of all accessions, when appearing as best hits in BLAST searches, would give a misleading $(9 \%)$ or incorrect $(7 \%)$ identification or would be unresolved (19\%). In terms of identification success, using a scoring system of categories $(1-3)=3,(4)=2,(5,6)=1$ and $(7)=0$, this would give a weighted average of $70.6 \%$ for query sequences having a high-scoring match to a given reference sequence (Table 1).

If all accessions would be updated with the adjusted identifications based on our analysis, the categories would change as follows: exact matches $=58 \%$, precise, but 


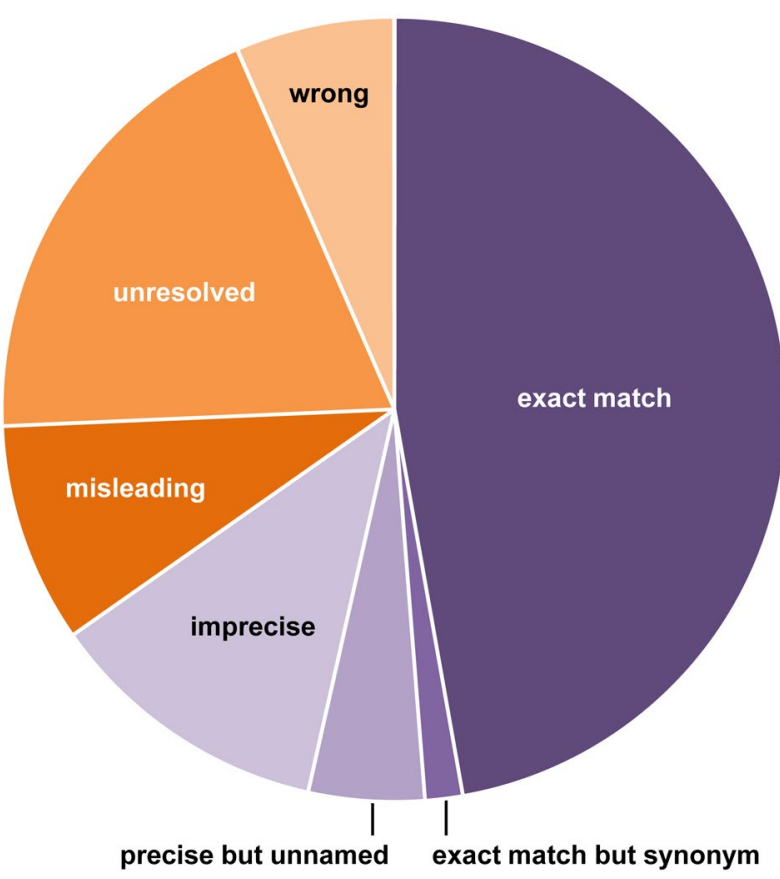

Figure 28. Proportions of categories of inferred matches and mismatches based on submitted sequence labels compared to updated species identifications.

as yet unnamed matches based on names with qualifiers (e.g., Usnea baileyi 2, U. aff. cornuta 1$)=31 \%$, and precise, but as yet unnamed matches (e.g., Usnea sp. 1 Wirtz $)=11 \%$. This would increase identification success to a weighted average of $99.0 \%$. Since third-party annotations to update sequence ID labels in primary repositories such as GenBank are not currently possible, we provide here an updated ITS alignment for the genus Usnea using the adjusted identification labels, which can be used in local BLAST approaches (File S8; Usnea Local BLAST Release 1.0). We also submitted the data to the curated UNITE database (Nilsson et al. 2018, 2019; Kõljalg et al. 2019). In addition, we provide an updated voucher table (Table S1).

ITS-based DNA barcoding in Usnea: inferred species richness

Based on the original identifications of the ITS accessions for Usnea s.lat., the 1,751 sequences nominally represented 143 taxa. Using our revised assessment, this number increased to at least 211 taxa, with 294 individual lineages that might in part represent additional taxa based on the detailed elaborations above (Table S1). Usnea s.lat. sequences in the curated UNITE database (Nilsson et al. 2018, 2019; Kõljalg et al. 2019) were resolved as 148 species hypotheses at the $98.5 \%$ threshold, 227 at the $99 \%$ threshold, and 322 at the $99.5 \%$ threshold [https://unite.ut.ee/search.php\#fndtn-panel1; Taxon name =Usnea (Genus)]. These numbers are just slightly above our assessment of taxa vs. lineages, suggesting that in Usnea s.lat., the $99 \%$ threshold denotes a conservative approach to species delimitation and the $99.5 \%$ threshold a progressive approach, the reality oscillating somewhere in between. Given these results, the often-used default threshold of $98.5 \%$ (Irinyi et al. 2015; Jeewon \& Hyde 2016; Kõljalg et al. 2019; Nilsson et al. 2019) would underestimate species richness in this group and thus our assessments using identity matrices based on this value may be too conservative.

Since metabarcoding pipelines often use a lower threshold of 97\% (Majaneva et al. 2015; Sinha et al. 2017; Anslan et al. 2018), we also computed the number of species hypotheses in Usnea s.lat. using this value, resulting in 56 taxa. This was only $26.5 \%$ of our likely estimate of 211 species and a gross underestimation of the real species richness in the genus.

If we assume that in the present data set, the number of previously recognized species more or less doubled based on phylogenetic resolution combined with available data on phenotype, the currently recognized number of nearly 450 taxa according to our checklist may actually correspond to up to 900 taxa, making Usnea an ultradiverse genus even when Dolichousnea and Eumitria are treated separately (Lücking et al. 2017a).

\section{Distribution patterns in Usnea: taxonomic versus phylogenetic inventories}

Based on our assessment of taxonomic inventories of the genus Usnea over the past 50 years (Table S2), we assigned each species to one of the following distribution patterns: (1) subcosmopolitan, (2) Pantropics, (3) amphi-Pacific, (4) amphi-Atlantic, (5) Gondwana (= amphi-Atlantic but tropical only, i.e. American and African tropics), (6) Afro-Eurasia, (7) Paleotropics, (8) Northern Hemisphere, (9) Southern Hemisphere, (10) Americas, (11), North and Central America, (12), Central and South America, (13) Russia-Asia, (14) Asia-Oceania, (15) North America, (16) Central America (incl. Mexico), (17) South America, (18) Europe, (19) Africa, (20) Asia, (21) Oceania, and (22) Antarctica. These were further classified into wide (1-5), contiguous (6-10), neighboring (11-14), and single regions (15-22).

Based on taxonomic inventories using phenotype-based identifications, a large proportion of the 438 species $(23 \%)$ were inferred to have wide distribution including across discontiguous areas, $8 \%$ a distribution in broad, contiguous areas consisting of more than two regions, $7 \%$ in two neighboring regions, and the majority $(62 \%)$ in a single region (Fig. 29). However, when species-level clades were considered, amounting to 286 entities in our ITS-based tree, distribution patterns were substantially much narrower with only $5 \%$ having a wide distribution, $12 \%$ a distribution in broad, contiguous areas, $1 \%$ in two neighboring regions, and $82 \%$ in a single region (Fig. 29).

When looking at individual distribution types, most types corresponding to wide distribution patterns substantially decreased when taking into account phylogenetically defined species, with the exception of amphi-Atlantic (Fig. 30). In contrast, several contiguous distribution types increased in proportion, namely Afro-Eurasia, Northern Hemisphere, and Southern Hemisphere (Fig. 30). All neighboring distribution types also decreased, whereas 


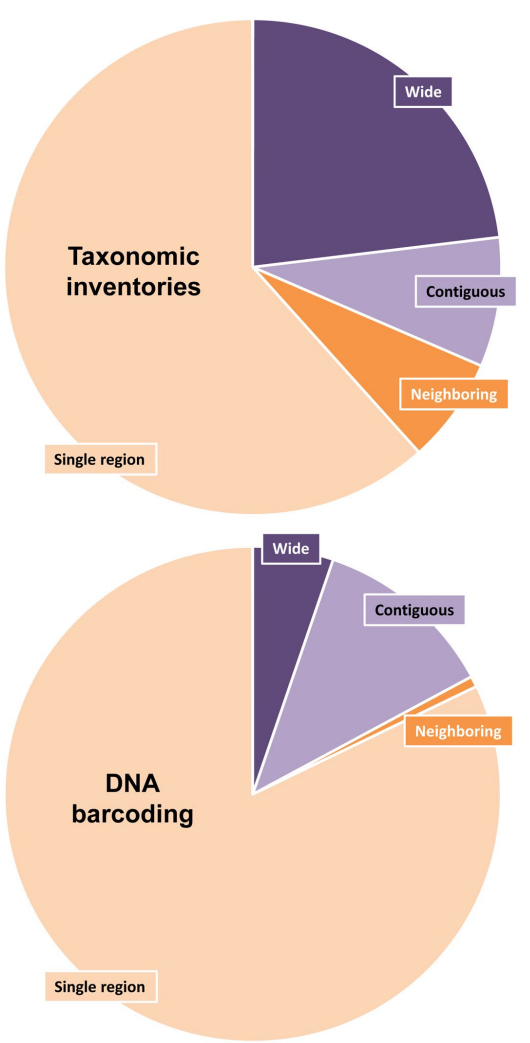

Figure 29. Proportion of principal distribution patterns when species are delimited by phenotype (traditional taxonomic inventories) vs. delimitation by molecular data (DNA barcoding).

single region distribution types mostly increased, with the exception of Central America (incl. Mexico) and Africa (Fig. 30). While some of this is due to taxonomic and geographic sampling bias, it became obvious that wide, discontiguous distribution patterns inferred through traditional species concepts are much less frequent than previously presumed, whereas wide distributions following contiguous areas are more frequent when species concepts are adjusted based on molecular data. Also, regional endemism is more frequent.

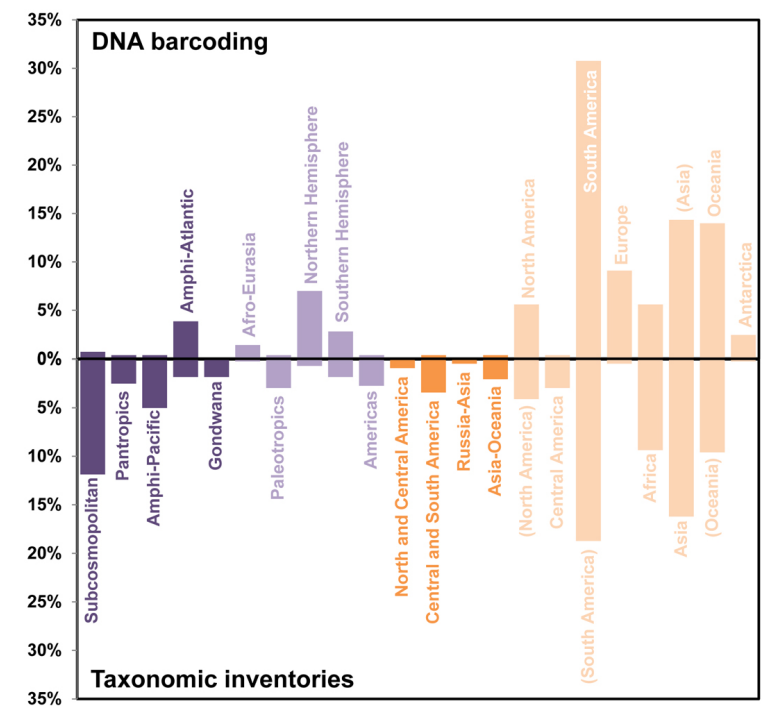

Figure 30. Proportion of individual distribution patterns when species are delimited by phenotype (traditional taxonomic inventories) vs. delimitation by molecular data (DNA barcoding).
ITS-based barcoding in Usnea compared to other lichenized genera

ITS-based barcoding as a means to delimit and recognize species has been amply used in other large genera of lichenized fungi, generally with good success. Notably, ITS provides generally higher levels of resolution and support in basidiolichens, including in the backbone, as exemplified by the genera Cora Fr. and Sulzbacheromyces B.P. Hodk. \& Lücking, often to the point that unambiguous alignment of the ITS within a genus becomes difficult (Lücking et al. 2017b; Coca et al. 2018). Among ascolichens, sizable monophyletic genera with a large amount of ITS data include Cladia Nyl., Cladonia P. Browne, Melanelia Ess., Parmelia Ach., Parmotrema A. Massal., Peltigera Willd., Pseudocyphellaria Vain., Ramalina Ach., and Sticta (Schreb.) Ach., and Strigula Fr.

Studies testing the utility of DNA barcoding markers often focus on the barcoding gap, with good success in genera of Parmeliaceae, such as Melanelia and relatives, Parmelia, and Parmotrema (Leavitt et al. 2014, 2016; Divakar et al. 2016; Del-Prado et al. 2019), but also in the genus Cladia (Parnmen et al. 2013). In other lineages, such as Cladonia, assessing the barcoding gap rendered ITS suboptimal compared to other markers (Kelly et al. 2011; Pino-Bodas et al. 2013; Kanz et al. 2015; Marthinsen et al. 2019). The barcoding gap approach may suffer from sampling bias and also may not reflect accurate phylogenetic relationships, as it is based on pairwise comparisons of sequence similarity (Lücking et al. 2020a, b), and it may be biased when underlying species concepts to not reflect phylogenetically delimited lineages (Marthinsen et al. 2019). The computation of barcoding gaps should therefore always be based on phylogenetically delimited lineages and not on phenotypically identified specimens.

When being used in broad, multiple alignment-based context, as here in the genus Usnea, ITS generally works well, although in recently evolving species complexes it may pose problems of resolution. Examples include the recently emended Peltigeraceae (Kraichak et al. 2018; Lücking 2019), for instance the genera Peltigera, Pseudocyphellaria and Sticta (Moncada et al. 2014; Magain \& Sérusiaux 2015; Lücking et al. 2017c; Magain et al. 2018: Ranft et al. 2018; Simon et al. 2018). Microlichens in the genus Strigula (Dothideomycetes) exhibit high levels of resolution with ITS and good congruence with other markers (Jiang et al. 2016, 2017a, b; Ford et al. 2019; Woo et al. 2020).

In contrast to some other fungal lineages (Lücking et al. 2020a), genome-wide analyses have also shown that intragenomic variation in the ITS is generally low, both in ascolichens (e.g., Rhizoplaca; Bradshaw et al. 2020) and basidiolichens (e.g., Cora; Lücking et al. 2014). Likewise, using a cloning approach, Saag et al. (2014) screened for potential ITS paralogs in Vulpicida and did not find evidence for paralogous copies of this marker. Thus, the main issue with ITS, at least in lichenized lineages, appears to be the occasional lack of resolution. Candidate cases, such as the Usnea antarctica / U. aurantiacoatra species pair (Grewe et al. 2018; Lagostina et al. 2018), 
are not only found in other groups of Usnea (U. florida vs. U. subfloridana, U. lambii vs. U. perpusilla), but morphologically discrete taxa not resolved by ITS are also known from other macrolichens, including Sticta fuliginosa (Dicks.) Ach. vs. S. limbata (Sm.) Ach. or S. filix (Sw.) Nyl. vs. S. lacera (Hook. f. \& Taylor) Müll. Arg. (Magain \& Sérusiaux 2015; Ranft et al. 2018; Moncada et al. 2020b). Besides resource-intensive methods such as microsatellite markers and RADseq, IGS appears to be a promising secondary barcoding marker in Usnea, but has also proven useful in other lichenized and non-lichenized fungal lineages, such as Thamnolia Ach. ex Schaer. and Schizophyllum Fr. (James et al. 2001; Onuţ-Brännström et al. 2017).

\section{Conclusions}

The fungal ITS barcoding marker has been used for about two decades to assess species delimitation and recognition in the genus Usnea. As in other lichenized and non-lichenized fungi, ITS has contributed substantially to unravel and remedy artificial taxonomic concepts and has helped to reshape taxonomy in the genus Usnea. However, the large amount of available data showed that ITS-based DNA barcoding in this genus also has its limits, both practically and conceptually.

In practical terms, while sequence quality was overall excellent and we found only few low-quality accessions, information on voucher specimens and overall identifications were inadequate in many cases. Particularly these two issues greatly challenge successful ITS-based barcoding in Usnea, as more often than not, the taxon identifications on BLAST hits will not indicate the correct species. This can be largely mitigated by updating and correcting voucher information. However, since this is not possible directly in primary repositories such as GenBank, we have here provided an updated alignment that can be used in local BLAST applications or through the curated UNITE ITS database. For future voucher information in Usnea (and corresponding cases), we strongly recommend listing key characters such as medullary chemistry in voucher tables, as has been done in a number of exemplar studies.

Another practical challenge to successful DNA barcoding is taxonomic and geographic coverage. Currently, only around $30 \%$ of the nearly 450 currently accepted species in Usnea s.lat. have been sequenced, and there is both taxonomic and geographic bias. Thus, while Dolichousnea and subgenus Neuropogon are well-represented among the data, sampling for Eumitria and Usnea s.str. remains moderate to poor and biased towards certain lineages. Geographically, Antarctica and Europe are well-represented, followed by South America, whereas North America, Africa, Asia and Oceania are undersampled. A peculiar situation arises with New Zealand, which is represented by a large amount of ITS accessions from across both major islands, but most of them left unidentified.

As outlined above, the species pair Usnea antarctica vs. U. aurantiacoatra is one example of an extremely well-sampled clade. Numerous ITS sequences were generated both from taxonomic and from ecological studies, demonstrating the ITS to be quite uniform in this clade, regardless of the study source. This underlines the reproducibility of this approach, regardless of the study and its objectives. On the other hand, analysis of highly resolving microsatellite and RADseq markers showed that ITS was not able to properly resolve the two species present in this complex.

While lack of resolution appears to be an issue with ITS in recently evolving species complexes in the genus Usnea, we did not find any evidence for gene duplication (paralogs) or hybridization causing artifactual clades, thus confirming aformentioned studies in Parmeliaceae (Vulpicida) or Lecanoraceae (Rhizoplaca). Comparison with other markers demonstrated that particularly IGS and RPB1 are useful to complement ITS-based phylogenies. IGS shows better resolution and support at species level, whereas $R P B 1$ has less terminal resolution and delineates larger species complexes. Both IGS and RPBI also provide better backbone resolution and support than ITS. IGS is therefore a promising secondary barcoding marker in Usnea, a notion matching aformentioned studies in other Parmeliaceae. The nuLSU marker appears to be of limited use in Usnea, providing neither good resolution nor good backbone support. The other three commonly employed protein-coding markers, TUB2, RPB2, and $M C M 7$, showed variable evidence of aberrant topologies here interpreted as paralog, particularly in the MCM7, and these markers should be used with great care, especially when it comes to multimarker coalescence species delimitation approaches.

Further challenges include difficult morphospecies that do not form coherent clades in ITS or other markers, suggesting various levels of cryptic speciation. The most notorious example is the Usnea cornuta complex discussed in detail above. In these cases, multimarker approaches using ITS, IGS and RPBI will help to assess supported lineages, but it cannot always be expected that these lineages will be readily separable using phenotypic characters.

Overall, we conclude that ITS is a good first approximation to assess species delimitation and recognition in the genus Usnea, as long as data are carefully analyzed and reference sequences are critically assessed and not taken at face value. In difficult groups, we recommend sequencing IGS as a secondary barcode marker, which together with ITS should result in reliable species assessments in most cases. However, additional studies using RADseq should be executed to assess the performance of the ITS-IGS barcoding approach in species complexes. In the near future, attempts should be made to close taxonomic and geographic gaps, in particular in Eumitria and Usnea s.str. and in the highly diverse areas of North America, Africa, Asia, and Oceania.

\section{Acknowledgements}

First and foremost, we take this opportunity to thank our esteemed colleague, mentor and friend Philippe Clerc, for his invaluable and continuous contributions to the taxonomy and systematics of the genus Usnea during the last 36 years. The present study, which we dedicate to Philippe on the occasion 
of his well-deserved retirement, reflects his influence and his work on many levels, including transferring his knowledge to numerous colleagues and students. We also thank Nora Wirtz for providing voucher data on samples of Neuropogon s.lat. from her studies. Yoshihito Ohmura placed the photograph of Dolichousnea longissima at our disposal. Andreas Beck helped with information about annotations of some type specimens housed in $\mathrm{M}$ and James Lendemer shared some insight into the taxonomy of the Usnea strigosa complex. Henrik Nilsson assisted with integrating the data set into UNITE. We thank Steve Leavitt and Yoshihito Ohmura for valuable comments that helped to improve the manuscript.

\section{Supplementary electronic material}

Figure S1. Global ITS-based, best-scoring maximum likelihood tree for the genus Usnea based on GenBank accessions, using original identifications. Download file

Figure S2. Global nuLSU-based, best-scoring maximum likelihood tree for the genus Usnea based on GenBank accessions, using original identifications. Download file

Figure S3. Global IGS-based, best-scoring maximum likelihood tree for the genus Usnea based on GenBank accessions, using original identifications. Download file

Figure S4. Global TUB2-based, best-scoring maximum likelihood tree for the genus Usnea based on GenBank accessions, using original identifications. Download file

Figure S5. Global $R P B$ 1-based, best-scoring maximum likelihood tree for the genus Usnea based on GenBank accessions, using original identifications. Download file

Figure S6. Global RPB2-based, best-scoring maximum likelihood tree for the genus Usnea based on GenBank accessions, using original identifications. Download file

Figure S7. Global MCM7-based, best-scoring maximum likelihood tree for the genus Usnea based on GenBank accessions, using original identifications. Download file

Table S1. Updated, complete voucher table for ITS accessions of the genus Usnea, including original and updated identifications and voucher information. Additional recent accessions for RBP1 and MCM7 corresponding to the studies by Araujo (2016) and Gerlach et al. (2017, 2019a, 2020) are also given. Download file

Table S2. Preliminary working checklist of regional and country/territory reports of species of Usnea, along with an assessment of currently establish taxonomic synonyms. Selected infraspecific names are included, simplified by combining the species and infraspecies epithets with a hyphen. TYPE indicates records corresponding to type citations (country of origin), including for taxonomic synonyms. Some reports by Dodge from Africa $(1956,1957)$ have not been verified as they are not treated in the various papers by Swinscow \& Krog $(1974,1975$, 1976a, b, 1978, 1979, 1988), and they have not been counted towards the total; potentially they may represent up to 27 additional species, but most of the other names established by Dodge $(1956,1957)$ have been subsumed under synonymy of other species (Swinscow \& Krog 1974, 1975, 1976a, b, 1978, 1979, 1988). Two unpublished names [ined.] have also not been included in the total count, leaving 447 accepted names (428 species and 19 infraspecies). Download file

File S1. Global ITS alignment for the genus Usnea based on GenBank accessions, using original identifications. Download file

File S2. Global nuLSU alignment for the genus Usnea based on GenBank accessions, using original identifications. Download file

File S3. Global IGS alignment for the genus Usnea based on GenBank accessions, using original identifications. Download file

File S4. Global TUB2 alignment for the genus Usnea based on GenBank accessions, using original identifications. Download file
File S5. Global RPB1 alignment for the genus Usnea based on GenBank accessions, using original identifications. Download file

File S6. Global RPB2 alignment for the genus Usnea based on GenBank accessions, using original identifications. Download file

File S7. Global MCM7 alignment for the genus Usnea based on GenBank accessions, using original identifications. Download file

File S8. Global ITS alignment for the genus Usnea based on GenBank accessions, using updated identifications (Usnea Local BLAST Release 1.0). Download file

\section{References}

Abbott, R. J., Barton, N. H. \& Good, J. M. 2016. Genomics of hybridization and its evolutionary consequences. Molecular Ecology 25: $2325-2332$.

Acharius, E. 1803. Methodus Qua Omnes Detectos Lichenes Secundum Organa Carpomorpha ad Genera, Species et Varietates Redigere atque Observationibus Illustrare Tentavit Erik Acharius. Stockholm.

Agassiz, L. 1850. Lake Superior: Tts Physical Character, Vegetation and Animals. With a narrative of the Tour by J. Elliot Cabot. Boston.

Ahti, T., Mayrhofer, H., Schultz, M., Tehler, A. \& Fryday, A. M. 2016. First supplement to the lichen checklist of South Africa. Bothalia 46: $1-8$.

Ait Hammou, M., Miara, M. D., Rebbas, K., Slimani, A, Ravera, S. \& Hamer El Ain, A. S. 2014. Mise à jour de l'inventaire des lichens d'Algérie. Revue Ecologie-Environment 10: 75-103.

Andreev, M., Kotlov, Y. \& Makarova, I. 1996. Checklist of lichens and lichenicolous fungi of the Russian Arctic. The Bryologist 99: 137-169.

Anslan, S., Nilsson, R. H., Wurzbacher, C., Baldrian, P., Tedersoo, L. \& Bahram, M. 2018. Great differences in performance and outcome of high-throughput sequencing data analysis platforms for fungal metabarcoding. MycoKeys 39: 29-40.

Aptroot, A. 2002. New and interesting lichens and lichenicolous fungi in Brazil. Fungal Diversity 9: 15-45.

Aptroot, A. 2016. Preliminary checklist of the lichens of Madagascar, with two new thelotremoid Graphidaceae and 131 new records. Willdenowia 46: 349-365.

Aptroot, A. \& Feijen, F. J. 2002. Annotated checklist of the lichens and lichenicolous fungi of Bhutan. Fungal Diversity 11: 21-48.

Aptroot, A. \& Iqbal, S. H. 2010. Annotated checklist of the lichens of Pakistan, with reports of new records. Herzogia 25: 211-229.

Aptroot, A. \& Seaward, M. R. 1999. Annotated checklist of Hongkong lichens. Tropical Bryology 17: 57-102.

Aptroot, A. \& Sparrius, L. B. 2006. Additions to the lichen flora of Vietnam, with an annotated checklist and bibliography. The Bryologist 109: 358-371.

Araujo, E. 2016. Sistemática Integrada del Género Usnea Dill. ex Adans. (Parmeliaceae) en la Península Ibérica. Doctoral Thesis, Universidad Complutense de Madrid, Spain.

Arcadia, L. 2013. Usnea dasopoga, a name to be reinstated for U. filipendula, and its orthography. Taxon 62: 604-605.

Armaleo, D. \& Clerc, P. 1991. Lichen chimeras: DNA analysis suggests that one fungus forms two morphotypes. Experimental Mycology 15: $1-10$.

Articus, K. 2004. Neuropogon and the phylogeny of Usnea sl (Parmeliaceae, Lichenized Ascomycetes). Taxon 53: 925-934.

Articus, K., Mattsson, J. E., Tibell, L., Grube, M. \& Wedin, M. 2002. Ribosomal DNA and $\beta$-tubulin data do not support the separation of the lichens Usnea florida and U. subfloridana as distinct species. Mycological Research 106: 412-418.

Arup, U., Ekman, S., Grube, M., Mattsson, J.-E. \& Wedin, M. 2007. The sister group relation of Parmeliaceae (Lecanorales, Ascomycota). Mycologia 99: 42-49. 
Asahina, Y. 1956. Lichens of Japan. Vol. III. Genus Usnea. Research Institute for Natural Resources, Tokyo.

Asahina, Y. 1968. Lichenologische Notizen (211-212). Journal of Japanese Botany 43: 495-499.

Asahina, Y. 1970. Lichenologische Notizen (235). On some Formosan Usnea with terminal peltate apothecia. Journal of Japanese Botany 45: 129-133.

Atalay, F., Halici, M. B., Mavi, A., Cakir, A., Odabaşoğlu, F., Kazaz, C., Aslan, A. \& Küfrevioğlu, Ö. İ. 2011. Antioxidant phenolics from Lobaria pulmonaria (L.) Hoffm. and Usnea longissima Ach. lichen species. Turkish Journal of Chemistry 35: 647-661.

Awasthi, G. 1986. Lichen genus Usnea in India. Journal of the Hattori Botanical Laboratory 61: 333-421.

Aydin, S., Kinalioğlu, K. \& Sökmen, B. B. 2018. Antioxidant, anti-urease and anti-elastase activities of Usnea longissima Ach. Bangladesh Journal of Botany 47: 429-435.

Azami, N., Seriñá, E. \& Arroyo, R. 2004. The Usnea species of Morocco in R.-G. Werner's herbarium. The Bryologist 107: 180-188.

Babington, C. 1852. Lichenes Himalayenses: being an Enumeration of the Lichens collected in the Himalaya Mountains etc. during the years 1847 and 1848. Hooker Journal of Botany 4: 243-253.

Badotti, F., Camargos-Fonseca, P. L., Ribeiro-Tome, L. M., Nunes, D. T. \& Góes-Neto, A. 2018. ITS and secondary biomarkers in fungi: review on the evolution of their use based on scientific publications. Brazilian Journal of Botany 41: 471-479.

Bakar, S. Z. A., Latip, J., bin Din, L. \& Samsuddin, M. W. 2014. Metabolic fingerprinting of lichen Usnea baileyi by Fourier transform infrared spectroscopy. AIP Conference Proceedings 1614: 452-455.

Behera, B. C., Mahadik, N. \& Morey, M. 2012. Antioxidative and cardiovascular-protective activities of metabolite usnic acid and psoromic acid produced by lichen species Usnea complanata under submerged fermentation. Pharmaceutical Biology 50: 968-979.

Bidartondo, M. I. 2008. Preserving accuracy in GenBank. Science 319: 1616.

Bjerke, J. W., Elvebakk, A. \& Elverland, E., 2006. The lichen genus Usnea in Norway north of the Arctic Circle: biogeography and ecology. Nova Hedwigia 83: 293-310.

Bokhorst, S., Convey, P., Huiskes, A. \& Aerts, R. 2016. Usnea antarctica, an important Antarctic lichen, is vulnerable to aspects of regional environmental change. Polar Biology 39: 511-521.

Boluda, C. G., Rico, V. J., Divakar, P. K., Nadyeina, O., Myllys, L., McMullin, R. T., Zamora, J. C., Scheidegger, C. \& Hawksworth, D. L. 2019. Evaluating methodologies for species delimitation: the mismatch between phenotypes and genotypes in lichenized fungi (Bryoria sect. Implexae, Parmeliaceae). Persoonia 42: 75-100.

Boom, B. M., Buck, W. R., Gracie, C. A. \& Tulig, M. 2009. Plants and Lichens of St. Eustatius. http://sweetgum.nybg.org/st_eustatius/ index.php

Bradshaw, M., Grewe, F., Thomas, A., Harrison, C. H., Lindgren, H., Muggia, L., St. Clair, L. L., Lumbsch, H. T., Leavitt, S. D. 2020. Characterizing the ribosomal tandem repeat and its utility as a DNA barcode in lichen-forming fungi. BMC Evolutionary Biology 20: 2.

Bruns, T. D., White, T. J. \& Taylor, J. W. 1991. Fungal molecular systematics. Annual Review of Ecology and Systematics 22: 525-564.

Buaruang, K., Boonpragob, K., Mongkolsuk, P., Sangvichien, E., Vongshewarat, K., Polyiam, W., Rangsiruji, A., Saipunkaew, W., Naksuwankul, K., Kalb, J. \& Parnmen, S. 2017. A new checklist of lichenized fungi occurring in Thailand. MycoKeys 23: 1-91.

Buckley, H. L., Rafat, A., Ridden, J. D., Cruickshank, R. H., Ridgway, H. J. \& Paterson, A. M. 2014. Phylogenetic congruence of lichenised fungi and algae is affected by spatial scale and taxonomic diversity. PeerJ 2014(1): e573-e573.

Bungartz, F., Truong, C., Herrera-Campos, M. A. \& Clerc, P. 2018. The genus Usnea (Parmeliaceae, Lecanoromycetes) in the Galapagos Islands. Herzogia 31: 571-629.
Burkin, A. A. \& Kononenko, G. P. 2015. Metabolites of toxigenic fungi in lichens of genera Alectoria, Bryoria, Evernia, Pseudevernia, and Usnea. Biology Bulletin 42: 296-301.

Bystrek, J. 1996. Usnea christhinae spec. nov. z Ameryki Poludniowej. Acta Mycologica 31: 171-174.

Cahyana, A. H., Suwarso, W. P. \& Nurdin, M. 2015. Isolation and structure elucidation of eumitrin A1 from lichen Usnea blepharea Motyka and its cytotoxic activity. International Journal of Pharmtech Research 8: 782-789.

Calvelo, S. \& Liberatore, S. 2002. Catálogo de los líquenes de la Argentina. Kurtziana 29: 7-170.

Canini, F., Geml, J., D‘Acqui, L. P., Selbmann, L., Onofri, S., Ventura, S. \& Zucconi, L. 2020. Exchangeable cations and pH drive diversity and functionality of fungal communities in biological soil crusts from coastal sites of Victoria Land, Antarctica. Fungal Ecology 45: 100923.

Cao, S., Zhang, J., Zheng, H., Liu, C. \& Zhou, Q. 2015. Photosynthetic performance in Antarctic lichens with different growth forms reflect the diversity of lichenized algal adaptation to microhabitats. Polish Polar Research 36: 175-188.

Cao, S., Zheng, H., Cao, Y., Liu, C., Zhu, L., Peng, F. \& Zhou, Q. 2018. Morphological differentiation and phylogenetic homogeneity in Usnea aurantiaco-atra reveal the complexity of lichen symbiosis. Polish Polar Research 39: 313-328.

Carrera, M. F. \& Carreras, H. A. 2011. Efectos de la aplicación de glifosato sobre parámetros químico-fisiológicos en Usnea amblyoclada [Müll. Arg.] Zahlbr. Ecología Austral 21: 353-361.

Ceker, S., Orhan, F., Kizil, H. E., Alpsoy, L., Gulluce, M., Aslan, A. \& Agar, G. 2015. Genotoxic and antigenotoxic potentials of two Usnea species. Toxicology and Industrial Health 31: 990-999.

Chaker, A. F. 2016. Usnea cornuta Körb. and Usnea glabrescens (Vainio) Vainio sens. lat.: species to be protected in the peninsula of Edough (Algeria). Cellular and Molecular Biology 62: 69-73.

Chao, C.-D., Hsü, L.-W. \& Sun, Z.-M. 1975. Species Novae Usneae sinicae. Acta Phytotaxonomica Sinica 13: 90-107.

Clerc, P. 1984a. Contribution à la revision de la systématique des usneés (Ascomycotina, Usnea) d'Europe. I. - Usnea florida (L.) Wigg. emend. Clerc. Cryptogamie, Bryologie et Lichénologie 5: 333-360.

Clerc, P. 1984b. Usnea wirthii - a new species of lichen from Europe and North Africa. Saussurea 15: 33-36.

Clerc, P. 1987. Systematics of the Usnea fragilescens aggregate and its distribution in Scandinavia. Nordic Journal of Botany 7: 479-495.

Clerc, P. 1992. Some new or interesting species of the genus Usnea (lichenised Ascomycetes) in the British Isles. Candollea 47: 513-526.

Clerc, P. 1997. Notes on the genus Usnea Dill. ex Adanson. The Lichenologist 29: 209-215.

Clerc, P. 2004. Notes on the genus Usnea Adanson. II. Bibliotheca Lichenologica 88: 79-90.

Clerc, P. 2006. Synopsis of Usnea (lichenized Ascomycetes) from the Azores with additional information on the species in Macaronesia. The Lichenologist 38: 191-212.

Clerc, P. 2007. Usnea. In: Nash, T. H. III, Gries, C. \& Bungartz, F. (eds), Lichen Flora of the Greater Sonoran Desert Region, Vol. 3, pp. 302-335. Lichens Unlimited, Arizona State University, Tempe.

Clerc, P. 2011a. Notes on the genus Usnea Adanson (lichenized Ascomycota). III. Bibliotheca Lichenologica 106: 41-51.

Clerc, P. 2011b. Usnea. In: Thell, A. \& Moberg, R. (eds), Nordic Lichen Flora 4, pp. 107-127. Museum of Evolution, Uppsala University, Uppsala.

Clerc, P. 2016. Notes on the genus Usnea (lichenized Ascomycota, Parmeliaceae) IV. Herzogia 29: 403-411.

Clerc, P. \& Diederich, P. 1991. Usnea wirthii Clerc new to North America and the British Isles. The Lichenologist 23: 405-407. 
Clerc, P. \& Herrera-Campos, M. A. 1997. Saxicolous species of Usnea subgenus Usnea (lichenized ascomycetes) in North America. The Bryologist 100: 281-301.

Clerc, P. \& May, P. F. 2007. Usnea flammea (Lecanorales) new for North America. The Bryologist 110: 126-128.

Clerc, P. \& Naciri, Y. 2021. Usnea dasopoga (Ach.) Nyl. and U. barbata (L.) Wigg. (Ascomycetes, Parmeliaceae) are two different species: a plea for reliable identifications in molecular studies. The Lichenologist (in press).

Clerc, P. \& Otte, V. 2018. Usnea viktoriana (Ascomycota, Parmeliaceae), a new European taxon of the Usnea barbata-dasopoga group, with a key to the shrubby-subpendulous sorediate Usnea species in Europe. The Lichenologist 50: 513-527.

Çobanoğlu, G., Sesal, C., Açıkgöz, B. \& Karaltı, İ. 2016. Evaluation of antimicrobial activity of the lichens Physcia aipolia, Xanthoria parietina, Usnea florida, Usnea subfloridana and Melanohalea exasperata. Modern Phytomorphology 10: 19-24.

Coca, L. F., Lücking, R. \& Moncada, B. 2018. Two new, sympatric and semi-cryptic species of Sulzbacheromyces (Lichenized Basidiomycota, Lepidostromatales) from the Chocó Biogeographic Region in Colombia. The Bryologist 121: 297-305.

Conti, M. E., Finoia, M. G., Bocca, B., Mele, G., Alimonti, A. \& Pino, A. 2012. Atmospheric background trace elements deposition in Tierra del Fuego region (Patagonia, Argentina), using transplanted Usnea barbata lichens. Environmental Monitoring and Assessment 184: $527-538$.

Crisp, M. D. \& Chandler, G. T. 1996. Paraphyletic species. Telopea 6: 813-844.

Davydov, E. A. 2014. The first checklist of lichens, lichenicolous, and allied fungi of Altaisky krai (Siberia, Russia). Mycotaxon 127: 331. http://www.mycotaxon.com/resources/checklists/davydov-v127-checklist.pdf

Degtjarenko, P., Jüriado, I., Mandel, T., Tõrra, T., Saag, A., Scheidegger, C. \& Randlane, T. 2019. Microsatellite based genetic diversity of the widespread epiphytic lichen Usnea subfloridana (Parmeliaceae, Ascomycota) in Estonia: comparison of populations from the mainland and an island. MycoKeys 58: 27-45.

Degtjarenko, P., Tõrra, T., Mandel, T., Marmor, L., Saag, A., Scheidegger, C. \& Randlane, T. 2018. Unconstrained gene flow between populations of a widespread epiphytic lichen Usnea subfloridana (Parmeliaceae, Ascomycota) in Estonia. Fungal Biology 122: 731-737.

Del-Prado, R., Buaruang, K., Lumbsch, H. T., Crespo, A. \& Divakar, P. K. 2019. DNA sequence-based identification and barcoding of a morphologically highly plastic lichen forming fungal genus (Parmotrema, Parmeliaceae) from the tropics. The Bryologist 122: 281-291.

Diederich, P., Lücking, R., Aptroot, A., Sipman, H. J. M., Braun, U., Ahti, T. \& Ertz, D. 2017. New species and new records of lichens and lichenicolous fungi from the Seychelles. Herzogia 30: 182-236.

Divakar, P. K., Leavitt, S. D., Molina, M. C., Del-Prado, R., Lumbsch, H. T., Crespo, A. 2016 (2015). A DNA barcoding approach for identification of hidden diversity in Parmeliaceae (Ascomycota): Parmelia sensu stricto as a case study. Botanical Journal of the Linnean Society 180: 21-29.

Divakar, P. K., Crespo, A., Kraichak, E., Leavitt, S. D., Singh, G., Schmitt, I. \& Lumbsch, H. T. 2017. Using a temporal phylogenetic method to harmonize family-and genus-level classification in the largest clade of lichen-forming fungi. Fungal Diversity 84: 101-117.

Dodge, C. W. 1956. Some lichens of tropical Africa. II. Usnea. Annals of the Missouri Botanical Garden 43: 381-396.

Dodge, C. W. 1957. Some lichens of tropical Africa. II. Usnea. Annals of the Missouri Botanical Garden 44: 1-76.

Dorey, J. E., Hoffman, J. R., Martino, J. L., Lendemer, J. C. \& Allen, J. L. 2019. First record of Usnea (Parmeliaceae) growing in New York City in nearly 200 years. The Journal of the Torrey Botanical Society 146: 69-77.
Elix, J. A. \& McCarthy, P. M. 1998. Catalogue of the Lichens of the Smaller Pacific Islands. Bibliotheca Lichenologica 70: 1-361.

Elix, J. A. \& McCarthy, P. M. 2008. Checklist of Pacific Island Lichens. https://www.anbg.gov.au/abrs/lichenlist/PACIFIC_introduction.html

Elix, J. A., Wirtz, N. \& Lumbsch, H. T. 2007. Studies on the chemistry of some Usnea species of the Neuropogon group (Lecanorales, Ascomycota). Nova Hedwigia 85: 491-501.

Esslinger, T. L. 2019. A cumulative checklist for the lichen-forming, lichenicolous and allied fungi of the continental United States and Canada, version 23. Opuscula Philolichenum 18: 102-378.

Fanovich, M. A., Ivanovic, J., Zizovic, I., Misic, D. \& Jaeger, P. 2016. Functionalization of polycaprolactone/hydroxyapatite scaffolds with Usnea lethariiformis extract by using supercritical $\mathrm{CO}_{2}$. Materials Science and Engineering C 58: 204-212.

Fée, A. L. A. 1825. Essai sur les Cryptogames des Écorces Exotiques Officinales. Didot, Paris.

Feibelman, T., Bayman, P. \& Cibula, W. G. 1994. Length variation in the internal transcribed spacer of ribosomal DNA in chanterelles. Mycological Research 98: 614-618.

Ford, M., Blanchon, D. J., Veale, A., Doyle, E. J., Rolfe, J. R. \& De Lange, P. J. 2019. Hidden in plain sight - a new Strigula species segregated from Strigula novae-zelandiae (Lichenized Ascomycota: Strigulaceae). Phytotaxa 424: 267-281.

Fos, S. \& Clerc, P. 2000. The lichen genus Usnea on Quercus suber in Iberian cork-oak forests. The Lichenologist 32: 67-88.

Fryday, A. M. \& Øvstedal, D. O. 2012. New species, combinations and records of lichenized fungi from the Falkland Islands (Islas Malvinas). The Lichenologist 44: 483-500.

Fryday, A. M. 2015. A new checklist of lichenised, lichenicolous and allied fungi reported from South Africa. Bothalia 45: 59-122.

Fryday, A. M., Orange, A., Ahti, T., Øvstedal, D. O. \& Crabtree, D. E. 2019. An annotated checklist of lichen-forming and lichenicolous fungi reported from the Falkland Islands (Islas Malvinas). Glalia 8: $1-100$.

Funk, D. J. \& Omland, K. E. 2003. Species-level paraphyly and polyphyly: frequency, causes, and consequences, with insights from animal mitochondrial DNA. Annual Review of Ecology, Evolution, and Systematics 34: 397-423.

Funk, E. R., Adams, A. N., Spotten, S. M., Van Hove, R. A., Whittington, K. T., Keepers, K. G., Pogoda, C. S., Lendemer, J. C., Tripp, E. A. $\&$ Kane, N. C. 2018. The complete mitochondrial genomes of five lichenized fungi in the genus Usnea (Ascomycota: Parmeliaceae). Mitochondrial DNA Part B 3: 305-308.

Gadea, A., Le Pogam, P., Biver, G., Boustie, J., Le Lamer, A.C., Le Dévéhat, F. \& Charrier, M. 2017. Which specialized metabolites does the native subantarctic gastropod Notodiscus hookeri extract from the consumption of the lichens Usnea taylorii and Pseudocyphellaria crocata? Molecules 22: 425.

Gagarina, L. V., Poryadina, L. N., Chesnokov, S. V. \& Konoreva, L. A. 2017. The lichen genus Usnea Dill. ex Adans. in the Sakha Republic (Yakutia). Botanica Pacifica 6: 31-36.

Galinato, M. G. M., Mangubat, C. B., Leonor, D. S., Cababa, G. R. C., Cipriano, B. P. S. \& Santiago, K. A. A. 2017. Identification and diversity of the fruticose lichen Usnea in Kalinga, Luzon Island, Philippines. Current Research in Environmental \& Applied Mycology 7: 249-257.

Galinato, M. G. M., Baguinon, J. R. C. \& Santiago, K. A. A. 2018. Review of the lichen genus Usnea in the Philippines. Studies in Fungi 3: 39-48.

Galloway, D. J. 2007. Flora of New Zealand Lichens. Revised Second Edition Including Lichen-Forming and Lichenicolous Fungi. Volumes 1 and 2. Manaaki Whenua Press, Lincoln, New Zealand.

Gargas, A. \& Taylor, J. W. 1995. Phylogeny of Discomycetes and early radiations of the apothecial Ascomycotina inferred from SSU rDNA sequence data. Experimental Mycology 19: 7-15. 
Gargas, A., DePriest, P. T., Grube, M. \& Tehler, A. 1995. Multiple origins of lichen symbioses in fungi suggested by SSU rDNA phylogeny. Science 268: 1492-1495.

Garnica, S., Schön, M. E., Abarenkov, K., Riess, K., Liimatainen, K., Niskanen, T., Dima, B., Soop, K., Frøslev, T. G., Jeppesen, T. S. \& Peintner, U. 2016. Determining threshold values for barcoding fungi: lessons from Cortinarius (Basidiomycota), a highly diverse and widespread ectomycorrhizal genus. FEMS Microbiology Ecology 92(4): fiw045.

Gerlach, A. C. L., Clerc, P. \& Silveira, R. M. B. 2017. Taxonomy of the corticolous, shrubby, esorediate, neotropical species of Usnea Adans. (Parmeliaceae) with an emphasis on southern Brazil. The Lichenologist 49: 199-238.

Gerlach, A. C. L., Toprak, Z., Naciri, Y., Caviró, E. A., Silveira, R. M. B. \& Clerc, P. 2019a. New insights into the Usnea cornuta aggregate (Parmeliaceae, lichenized Ascomycota): Molecular analysis reveals high genetic diversity correlated with chemistry. Molecular Phylogenetics and Evolution 131: 125-137. [Available online 30 October 2018]

Gerlach, A. C. L., Silveira, R. M. B. \& Clerc, P. 2019b. Usnea oreophila (Parmeliaceae), a new saxicolous species from the mountains of Brazil. The Bryologist 122: 122-129.

Gerlach, A, Silveira, R. M. B., Rojas, C. \& Clerc, P. 2020. Naming and describing the diversity in the Usnea cornuta aggregate (lichenized Ascomycota, Parmeliaceae) focusing on Brazilian specimens. Plant and Fungal Systematics 65: 272-302.

Gilks, W. R., Audit, B., De Angelis, D., Tsoka, S. \& Ouzounis, C. A. 2002. Modeling the percolation of annotation errors in a database of protein sequences. Bioinformatics 18: 1641-1649.

Goffinet, B. \& Bayer, R. J. 1997. Characterization of mycobionts of photomorph pairs in the Peltigerineae (lichenized ascomycetes) based on internal transcribed spacer sequences of the nuclear ribosomal DNA. Fungal Genetics and Biology 21: 228-237.

Greenwood, S., Chen, J. C., Chen, C. T. \& Jump, A. S. 2016. Community change and species richness reductions in rapidly advancing tree lines. Journal of Biogeography 43: 2274-2284.

Grewe, F., Lagostina, E., Wu, H., Printzen, C. \& Lumbsch, H. T. 2018. Population genomic analyses of RAD sequences resolves the phylogenetic relationship of the lichen-forming fungal species Usnea antarctica and Usnea aurantiacoatra. MycoKeys 43: 91-113.

Groner, U. \& LaGreca, S. 1997. The 'Mediterranean' Ramalina panizzei north of the Alps: morphological, chemical and rDNA sequence data. The Lichenologist 29: 441-454.

Gruezo, W. S. 1979. Compendium of Philippine lichens. Kalikasan, Philippine Journal of Biology 8: 267-300.

Gumboski, E. L. \& Eliasaro, S. 2011. Checklist of lichenized fungi of Santa Catarina state (Brazil). Mycotaxon 115. 535. http://www. mycotaxon.com/resources/checklists/Gumboski-v115-checklist.pdf

Gunasekaran, S., Rajan, V. P., Ramanathan, S., Murugaiyah, V., Samsudin, M. W. \& Din, L. B. 2016. Antibacterial and antioxidant activity of lichens Usnea rubrotincta, Ramalina dumeticola, Cladonia verticillata and their chemical constituents. Malaysian Journal of Analytical Sciences 20: 1-13.

Hafellner, J. 2008. Additions and corrections to the checklist and Bibliography of Lichens and Lichenicolous Fungi of Insular Laurimacaronesia. IV. Fritschiana 64: 1-28.

Hale, M. E. 1962. The chemical strains of Usnea strigosa. The Bryologist 65: 291-294.

Hall, T. A. 1999. BioEdit: a user-friendly biological sequence alignment editor and analysis program for Windows 95/98/NT. Nucleic Acids Symposium Series 41: 95-98.

Hall, T. 2011. BioEdit: an important software for molecular biology. GERF Bulletin Biosci 2(1): 60-61.

Halonen, P. 2000. Usnea pacificana, sp. nov. and U. wasmuthii (lichenized Ascomycetes) in Pacific North America. The Bryologist 103: $38-43$.
Halonen, P., Clerc, P., Goward, T., Brodo, I. M. \& Wulff, K. 1998. Synopsis of the genus Usnea (lichenized Ascomycetes) in British Columbia, Canada. The Bryologist 101: 36-60.

Halonen, P., Myllys, L., Ahti, T. \& Petrova, O. V. 1999. The lichen genus Usnea in East Fennoscandia. III. The shrubby species. Annales Botanici Fennici 36: 235-256.

He, Y. \& Zhang, Z. 2012. Diversity of organism in the Usnea longissima lichen. African Journal of Microbiology Research 6: 4797-4804.

Hekking, W. H. A. \& Sipman, H. J. M. 1988. The lichens reported from the Guianas before 1987. Willdenowia 17: 193-228.

Hermansson, J. O. \& Thor, G. 2004. Byssoloma subdiscordans and Usnea substerilis new to Sweden. Graphis scripta 15: 42-44.

Herrera-Campos, M. A. 2016. Usnea in Mexico. Bibliotheca Lichenologica 110: 505-620.

Herrera-Campos, M. A., Clerc, P. \& Nash III, T. H. 1998. Pendulous species of Usnea from the temperate forests in Mexico. The Bryologist 101: 303-329.

Herrera-Campos, M. A., Nash III, T. H. \& Garcia, A. Z. 2001. Preliminary study of the Usnea fragilescens aggregate in Mexico. The Bryologist 104: 235-259.

Hinds, J. W. \& Hinds, P. L. 2007. The macrolichens of New England. Memoirs of the New York Botanical Garden 96: 1-584.

Hur, J. S., Wang, L. S., Oh, S. O., Kim, G. H., Lim, K. M., Jung, J. S. \& Koh, Y. J. 2005. Highland macrolichen flora of northwestern Yunnan, China. The Journal of Microbiology 43: 228-236.

Irinyi, L., Serena, C., Garcia-Hermoso, D., Arabatzis, M., Desnos-Ollivier, M., Vu, D., Cardinali, G., Arthur, I., Normand, A. C., Giraldo, A., da Cunha, K. C., Sandoval-Denis, M., Hendrickx, M., Nishikaku, A. S., de Azevedo Melo, A. S., Merseguel, K. B., Khan, A., Parente Rocha, J. A., Sampaio, P., da Silva Briones, M. R., Ferreira, R. C., de Medeiros Muniz, M., Castañón-Olivares, L. R., Estrada-Barcenas, D., Cassagne, C., Mary. C., Duan, S. Y., Kong, F., Sun, A. Y., Zeng, X., Zhao, Z., Gantois, N., Botterel, F., Robbertse, B., Schoch. C. L., Gams, W., Ellis, D., Halliday, C., Chen, S., Sorrell, T. C., Piarroux, R., Colombo, A. L., Pais, C., de Hoog, S., Zancopé-Oliveira, R. M., Taylor, M. L., Toriello, C., de Almeida Soares, C. M., Delhaes, L., Stubbe, D., Dromer, F., Ranque, S., Guarro, J., Cano-Lira, J. F., Robert, V., Velegraki, A. \& Meyer W. 2015. International Society of Human and Animal Mycology (ISHAM) - ITS reference DNA barcoding database - The quality controlled standard tool for routine identification of human and animal pathogenic fungi. Medical Mycology 53: 313-337.

James, T. Y., Moncalvo, J. M., Li, S. \& Vilgalys, R. 2001. Polymorphism at the ribosomal DNA spacers and its relation to breeding structure of the widespread mushroom Schizophyllum commune. Genetics 157: 149-161.

Jaouen, G., Sagne, A., Buyck, B., Decock, C., Louisanna, E., Manzi, S., Baraloto, C., Roy, M. \& Schimann, H. 2019. Fungi of French Guiana gathered in a taxonomic, environmental and molecular dataset. Scientific Data 6: 1-6.

Jayalal, U., Joshi, S., Oh, S. O., Koh, Y. J., Crişan, F. \& Hur, J. S. 2013. Lichen mycota in South Korea: the genus Usnea. Mycobiology 41: 126-130.

Jeewon, R. \& Hyde, K. D. 2016. Establishing species boundaries and new taxa among fungi: recommendations to resolve taxonomic ambiguities. Mycosphere 7: 1669-1677.

Jiang, S. H., Wei, X. L. \& Wei, J. C. 2016. Strigula sinoaustralis sp. nov. and three Strigula spp. new to China. Mycotaxon 131: 795-803.

Jiang, S. H., Wei, X. L. \& Wei, J. C. 2017a. Two new species of Strigula (lichenised Dothideomycetes, Ascomycota) from China, with a key to the Chinese foliicolous species. MycoKeys 19: 31-42.

Jiang, S. H., Wei, X. L. \& Wei, J. C. 2017b. A new species and two new records of Strigula (lichenized Ascomycota) from China. Mycoscience 58: 391-397.

Kalachar, H. C. B., Nayaka, Y. A., Vinayaka, K. S., Viswanatha, R. \& Vasanth Kumar, M. S. 2012. Electrochemical studies on usnic acid from Usnea pseudosinensis using multi walled carbon nanotube 
modified pencil graphite electrode. International Journal of Analytical and Bioanalytical Chemistry 2: 179-184.

Kanz, B., von Brackel, W., Cezanne, R., Eichler, M., Hohmann, M. L., Teuber, D. \& Printzen, C. 2015. DNA barcodes for the distinction of reindeer lichens: a case study using Cladonia rangiferina and C. stygia. Herzogia 28: 445-464.

Kasuga, T., Woods, C., Woodward, S. \& Mitchelson, K. 1993. Heterobasidion annosum 5.8s ribosomal DNA and internal transcribed spacer sequence: rapid identification of European intersterility groups by ribosomal DNA restriction polymorphism. Current Genetics 24: 433-436.

Katoh, K. \& Standley, D. M. 2013. MAFFT multiple sequence alignment software version 7: improvements in performance and usability. Molecular Biology and Evolution 30: 772-780.

Kelly, L. J., Hollingsworth, P. M., Coppins, B. J., Ellis, C. J., Harrold, P., Tosh, J. \& Yahr, R. 2011. DNA barcoding of lichenized fungi demonstrates high identification success in a floristic context. New Phytologist 191: 288-300.

Keon, D. B. \& Muir, P. S. 2002. Growth of Usnea longissima across a variety of habitats in the Oregon Coast Range. The Bryologist 105: 233-242.

Kim, J. H., Ahn, I. Y., Hong, S. G., Andreev, M., Lim, K. M., Oh, M. J., Koh, Y. J. \& Hur, J.-S., 2006. Lichen flora around the Korean Antarctic Scientific Station, King George Island, Antarctic. The Journal of Microbiology 44: 480-491.

Kõljalg, U., Abarenkov, K., Nilsson, R. H., Larsson, K. H. \& Taylor, A. F. 2019. The UNITE Database for Molecular Identification and for Communicating Fungal Species. Biodiversity Information Science and Standards 3:e37402.

Kozlov, A. M., Zhang, J., Yilmaz, P., Glöckner, F. O. \& Stamatakis, A. 2016. Phylogeny-aware identification and correction of taxonomically mislabeled sequences. Nucleic Acids Research 44: 5022-5033.

Kraichak, E., Crespo, A., Divakar, P. K., Leavitt, S. D. \& Lumbsch, H. T. 2017. A temporal banding approach for consistent taxonomic ranking above the species level. Scientific Reports 7: 1-7.

Kraichak, E., Huang, J. P., Nelsen, M., Leavitt, S. D. \& Lumbsch, H. T. 2018. A revised classification of orders and families in the two major subclasses of Lecanoromycetes (Ascomycota) based on a temporal approach. Botanical Journal of the Linnean Society 188: 233-249.

Krog, H. 1994. New observations on Usnea subgenus Eumitria in eastern and central Africa. In: Seyani, J. H. \& Chikuni, A. C. (eds), Proceedings of the XIIIth Plenary Meeting, pp. 813-821. AETFAT, Malawi.

Kroken, S. \& Taylor, J. W. 2001. A gene genealogical approach to recognize phylogenetic species boundaries in the lichenized fungus Letharia. Mycologia 93: 38-53.

Kuchta, S. R., Brown, A. D. \& Highton, R. 2018. Disintegrating over space and time: paraphyly and species delimitation in the Wehrle's Salamander complex. Zoologica Scripta 47: 285-299.

Kurtzman, C. P. 1985. Molecular taxonomy of the fungi. In: Bennett, J. W. \& Lasure, L. L. (eds), Gene Manipulations in Fungi, pp. 35-63. Academic Press, New York.

Kusaba, M. \& Tsuge, T. 1995. Phylogeny of Alternaria fungi known to produce host-specific toxins on the basis of variation in internal transcribed spacers of ribosomal DNA. Current Genetics 28: 491-498.

Lagostina, E., Dal Grande, F., Andreev, M. \& Printzen, C. 2018. The use of microsatellite markers for species delimitation in Antarctic Usnea subgenus Neuropogon. Mycologia 110: 1047-1057.

LaGreca, S. 1999. A phylogenetic evaluation of the Ramalina americana chemotype complex (lichenized Ascomycota, Ramalinaceae) based on rDNA ITS sequence data. The Bryologist 102: 602-618.

Laguna-Defior, C., Pintado, A., Green, T. A., Blanquer, J. M. \& Sancho, L. G. 2016. Distributional and ecophysiological study on the Antarctic lichens species pair Usnea antarctica/Usnea aurantiaco-atra. Polar Biology 39: 1183-1195.
Lamb, I. M. 1939. A review of the genus Neuropogon (Nees, Fl.) Nyl., with special reference to the antarctic species. Journal of the Linnean Society, Botany 52: 199-237.

Landvik, S., Eriksson, O. E. \& Berbee, M. L. 2001. Neolecta - a fungal dinosaur? Evidence from $\beta$-tubulin amino acid sequences. Mycologia 93: 1151-1163.

Leavitt, S. D., Esslinger, T. L., Divakar, P. K., Crespo, A. \& Lumbsch, H. T. 2016. Hidden diversity before our eyes: delimiting and describing cryptic lichen-forming fungal species in camouflage lichens (Parmeliaceae, Ascomycota). Fungal Biology 120: 1374-1391.

Leavitt, S. D., Esslinger, T. L., Hansen, E. S., Divakar, P. K., Crespo, A., Loomis, B. F. \& Lumbsch, H. T. 2014. DNA barcoding of brown Parmeliae (Parmeliaceae) species: a molecular approach for accurate specimen identification, emphasizing species in Greenland. Organisms Diversity and Evolution 14: 11-20.

Lee, J. S., Lee, H. K., Hur, J. S., Andreev, M. \& Hong, S. G. 2008. Diversity of the lichenized fungi in King George Island, Antarctica, revealed by phylogenetic analysis of partial large subunit rDNA sequences. Journal of Microbiology and Biotechnology 18: 1016-1023.

Lee, S. B. \& Taylor, J. W. 1992. Phylogeny of five fungus-like protoctistan Phytophthora species, inferred from the internal transcribed spacers of ribosomal DNA. Molecular Biology and Evolution 9: 636-653.

Lendemer, J. C. \& Tavares, I. I. 2003a. Nomenclature and typification in the genus Usnea (lichenized Ascomycetes) - I. Usnea rigida. Proceedings of the Academy of Natural Sciences of Philadelphia 153: $177-180$.

Lendemer, J. C. \& Tavares, I. I. 2003b. Nomenclature and typification in the genus Usnea (lichenized Ascomycetes) - II. Usnea fallax and Usnea paradoxa. Proceedings of the Academy of Natural Sciences of Philadelphia 153: 181-185.

Lendemer, J. C., Harris, R. C. \& Ruiz, A. M. 2016. A review of the lichens of the Dare regional biodiversity hotspot in the Mid-Atlantic Coastal Plain of North Carolina, eastern North America. Castanea 81: 1-77.

Lendemer, J. C., Keepers, K. G., Tripp, E. A., Pogoda, C. S., McCain, C. M. \& Kane, N. C. 2019. A taxonomically broad metagenomic survey of 339 species spanning 57 families suggests cystobasidiomycete yeasts are not ubiquitous across all lichens. American Journal of Botany 106: 1090-1095.

Li, Y., Kromer, B., Schukraft, G., Bubenzer, O., Huang, M. R., Wang, Z. M., Bian, L. G. \& Li, C. S. 2014. Growth rate of Usnea aurantiacoatra (Jacq.) Bory on Fildes Peninsula, Antarctica and its climatic background. PLoS One 9(6): e100735.

Li, Y., Yang, R. H., Jiang, L., Hu, X. D., Wu, Z. J. \& Yao, Y. J. 2017. rRNA pseudogenes in filamentous ascomycetes as revealed by genome data. Genes, Gemomes, Genetics 7: 2695-2703.

Lin, C. K. 2007. The lichen genus Usnea at Meifeng, central Taiwan. Collection and Research 20: 1-7.

Linder, C. R. \& Rieseberg, L. H. 2004. Reconstructing patterns of reticulate evolution in plants. American Journal of Botany 91: 1700-1708.

Lindner, D. L. \& Banik, M. T. 2011. Intragenomic variation in the ITS rDNA region obscures phylogenetic relationships and inflates estimates of operational taxonomic units in genus Laetiporus. Mycologia 103: 731-740.

Lohézic-Le Dévéhat, F., Tomasi, S., Elix, J. A., Bernard, A., Rouaud, I., Uriac, P. \& Boustie, J. 2007. Stictic acid derivatives from the lichen Usnea articulata and their antioxidant activities. Journal of Natural Products 70: 1218-1220.

Lohtander, K., Myllys, L., Sundin, R., Källersjö, M. \& Tehler, A. 1998. The species pair concept in the lichen Dendrographa leucophaea (Arthoniales): analyses based on ITS sequences. The Bryologist 101: 404-411.

Lucarini, R., Tozatti, M. G., de Oliveira Salloum, A. I., Crotti, A. E., Silva, M. L., Gimenez, V. M., Groppo, M., Januário, A. H., Martins, C. H. \& Cunha, W. R. 2012. Antimycobacterial activity of Usnea 
steineri and its major constituent (+)-usnic acid. African Journal of Biotechnology 11: 4636-4639.

Lücking, R. 2019. Stop the abuse of time! A critical review of temporal banding for rank-based classifications in Fungi (including lichens) and other organisms. Critical Review in Plant Sciences 38: 199-253.

Lücking, R., Lawrey, J. D., Gillevet, P. M., Sikaroodi, M., Dal Forno, M. \& Berger, S. A. 2014. Multiple ITS haplotypes in the genome of the lichenized basidiomycete Cora inversa (Hygrophoraceae): fact or artifact? Journal of Molecular Evolution 78: 148-162.

Lücking, R., Hodkinson, B. P. \& Leavitt, S. D. 2017a ('2016'). The 2016 classification of lichenized fungi in the Ascomycota and $\mathrm{Ba}$ sidiomycota - Approaching one thousand genera. The Bryologist 119: 361-416.

Lücking, R., Dal Forno, M., Moncada, B., Coca, L. F., Vargas-Mendoza, 1. Y., Aptroot, A., Arias, L. J., Besal, B., Bungartz, F., Cabrera-Amaya, D. M., Cáceres, M. E. S., Chaves, J. L., Eliasaro, S., Gutiérrez, M. C., Hernández-M., J. E., Herrera-Campos, M. A., Holgado-Rojas, M. E., Jonitz, H., Kukwa, M., Lucheta, F., Madriñán, S., Marcelli, M. P., Martins, S. M. A., Mercado-Díaz, J. A., Molina, J. A., Morales, E. A., Nelson, P. R., Nugra, F., Ortega, F., Paredes, T., Patiño, A. L., Peláez-Pulido, R. N., Pérez-Pérez, R. E., Perlmutter, G. B., Rivas-Plata, M. E., Robayo, J., Rodríguez, C., Simijaca, D. F., Soto-Medina, E., Spielmann, A. A., Suárez-Corredor, A., Torres, J. M., Vargas, C. A., Yánez-Ayabaca, A., Weerakoon, G., Wilk. K., Celis-Pacheco, M., Diazgranados, M., Brokamp, G., Borsch, T., Gillevet, P. M., Sikaroodi, M. \& Lawrey, J. D. 2017b. Turbo-taxonomy to assemble a megadiverse lichen genus: seventy new species of Cora (Basidiomycota: Agaricales: Hygrophoraceae), honouring David Leslie Hawksworth's seventieth birthday. Fungal Diversity 84: 139-207.

Lücking, R., Moncada, B., McCune, B., Farkas, E. E., Goffinet, B., Parker, D., Chaves, J. L., Lőkös, L., Nelson, P. R., Spribille, T., Stenroos, S., Wheeler, T., Yanez-Ayabaca, A., Dillman, K., Gockman, O. T., Goward, T., Hollinger, J., Tripp, E. A., Villella, J., Álvaro-Alba, W. R., Arango, C. J., Cáceres, M. E. S., Coca, L. F., Printzen, C., Rodríguez, C., Scharnagl, K., Rozzi, R., Soto-Medina, E. \& Yakovchenko, L. S. 2017c. Pseudocyphellaria crocata (Ascomycota: Lobariaceae) in the Americas reveals to be ten species, and none of them is Pseudocyphellaria crocata. The Bryologist 120: 441-500

Lücking, R., Aime, M. C., Robberts, B., Miller, A. N., Ariyawansa, H. A., Aoki, T., Cardinali, G., Crous, P. W., Druzhinina, I. S., Geiser, D. M., Hawksworth, D. L., Hyde, K. D., Irinyi, L., Jeewon, R., Johnston, P. R., Kirk, P. M., Malosso, E., May, T. W., Meyer, W., Öpik, M., Robert, V., Stadler, M., Thines, M., Vu, D., Yurkov, A. M., Zhang, N., Schoch, C. L. 2020a. Unambiguous identification of fungi: where do we stand and how accurate and precise is fungal barcoding? IMA Fungus 11: 14.

Lücking, R., Truong, B. V., Huong, D. T. T., Le, N. H., Nguyen, Q. D., Nguyen, V. D., Von Raab-Straube, E., Bollendorff, S., Govers. K. \& Di Vincenzo, V. 2020b. The caveats of fungal barcoding: a case study in the genus Trametes s.lat. (Basidiomycota: Polyporales) in Vietnam reveals multiple issues with mislabeled reference sequences and calls for third-party annotations. Willdenowia 50: 383-403.

Lücking, R., Moncada, B., Soto-Medina, E., Simijaca, D. \& Sipman, H. J. M. 2020c. Actualización nomenclatural y taxonómica al Catálogo de Líquenes de Colombia. Revista de la Academia Colombiana de Ciencias Exactas, Físicas y Naturales (in press).

Lukáč, M. 2010. Contribution to the genus Usnea (Parmeliaceae) in Slovakia III: apotheciate specimens of U. filipendula, U. florida, U. intermedia and U. subfloridana. Acta Facultatis Pharmaceuticae Universitatis Comenianae 57: 57-67.

Lumbsch, H. T. \& Wirtz, N. 2011. Phylogenetic relationships of the neuropogonoid core group in the genus Usnea (Ascomycota: Parmeliaceae). The Lichenologist 43: 553-559.

Lumbsch, H. T., Ahti, T., Altermann, S., Amo De Paz, G., Aptroot, A., Arup, U., Bárcenas Peña, A., Bawingan, P. A., Benatti, M. N., Betancourt, L., Björk, C. R., Boonpragob, K., Brand, M., Bungartz, F., Cáceres, M. E. S., Candan, M, Chaves, J. L., Clerc, P., Common, R., Coppins, B. J., Crespo, A., Dal Forno, M., Divakar, P. K., Duya,
M. V., Elix, J.A., Elvebakk, A., Fankhauser, J. D., Farkas, E., Ferraro, L. I., Fischer, E., Galloway, D. J., Gaya, E., Giralt, M., Goward, T., Grube, M., Hafellner, J., Hernández M., J. E., Herrera Campos, M. A., Kalb, K., Kärnefelt, I., Kantvilas, G., Killmann, D., Kirika, P., Knudsen, K., Komposch, H., Kondratyuk, S., Lawrey, J. D., Mangold, A., Marcelli, M. P., Mccune, B., Ines Messuti, M., Michlig, A., Miranda González, R., Moncada, B., Naikatini, A., Nelsen, M. P., Øvstedal, D. O., Palice, Z., Papong, K., Parnmen, S., Pérez-Ortega, S., Printzen, C., Rico, V. J., Rivas Plata, E., Robayo, J., Rosabal, D., Ruprecht, U., Salazar Allen, N., Sancho, L., Santos De Jesus, L., Santos Vieira, T., Schultz, M., Seaward, M. R. D., Sérusiaux, E., Schmitt, I., Sipman, H. J. M., Sohrabi, M., Søchting, U., Zeuthen Søgaard, M., Sparrius, L. B., Spielmann, A., Spribille, T., Sutjaritturakan, J., Thammathaworn, A., Thell, A., Thor, G., Thüs, H., Timdal, E., Truong, C., Türk, R., Umaña Tenorio, L., Upreti, D. K., Van Den Boom, P., Vivas Rebuelta, M., Wedin, M., Will-Wolf, S., Wirth, V., Wirtz, N., Yahr, R., Yeshitela, K., Ziemmeck, F., Wheeler, T. \& Lücking, R. 2011. One hundred new species of lichenized fungi: a signature of undiscovered global diversity. Phytotaxa 18: 1-127.

Magain, N. \& Sérusiaux, E. 2015. Dismantling the treasured flagship lichen Sticta fuliginosa (Peltigerales) into four species in Western Europe. Mycological Progress 14: 97.

Magain, N., Truong, C., Goward, T., Niu, D., Goffinet, B., Sérusiaux, E., Vitikainen, O., Lutzoni, F. \& Miadlikowska, J. 2018. Species delimitation at a global scale reveals high species richness with complex biogeography and patterns of symbiont association in Peltigera section Peltigera (lichenized Ascomycota: Lecanoromycetes). Taxon 67: 836-870.

Mahadik, N., Morey, M. V., Behera, B.C., Makhija, U. V. \& Naik, D. G. 2011. Cardiovascular-protective, antioxidative, and antimicrobial properties of natural thallus of lichen Usnea complanata. Latin American Journal of Pharmacy 30: 220-228.

Majaneva, M., Hyytiäinen, K., Varvio, S. L., Nagai, S. \& Blomster, J. 2015. Bioinformatic amplicon read processing strategies strongly affect eukaryotic diversity and the taxonomic composition of communities. PLoS One 10(6): e0130035.

Magnusson, A. H. 1940. Lichens from central Asia I. In: Hedin, S. (ed.). Reports from the Scientific Expedition to the North-Western Provinces of China under the Leadership of Dr. Sven Hedin. The Sino-Swedish Expedition. 13, XI. Botany, 1. Aktiebolaget Thule, Stockholm.

Marcano, V., Morales Méndez, A., Sipman, H. J. M. \& Calderon, L. 1996. A first checklist of the lichen-forming fungi of the Venezuelan Andes. Tropical Bryology 12: 193-235.

Marcelli, M. P. 1992. Ecologia Liquênica nos Manguezais do Sul-Sudeste Brasileiro. Bibliotheca Lichenologica 47: i-vii, 1-288.

Mark, K., Saag, L., Leavitt, S. D., Will-Wolf, S., Nelsen, M. P., Tõrra, T., Saag, A., Randlane, T. \& Lumbsch, H. T. 2016a. Evaluation of traditionally circumscribed species in the lichen-forming genus Usnea, section Usnea (Parmeliaceae, Ascomycota) using a six-locus dataset. Organisms Diversity \& Evolution 16: 497-524.

Mark, K., Cornejo, C., Keller, C., Flück, D. \& Scheidegger, C. 2016 b. Barcoding lichen-forming fungi using 454 pyrosequencing is challenged by artifactual and biological sequence variation. Genome 59: 685-704.

Marthinsen, G., Rui, S. \& Timdal, E. 2019. OLICH: A reference library of DNA barcodes for Nordic lichens. Biodiversity Data Journal 7: e36252.

Mattsson, J. E. \& Wedin, M. 1998. Phylogeny of the Parmeliaceae - DNA data versus morphological data. The Lichenologist 30: $463-472$.

Maulidiyah, M., Cahyana, A. H. \& Suwarso, W. P. 2011. A new phenolic compound from acetone extract of lichen Usnea flexuosa Tayl. Indonesian Journal of Chemistry 11: 290-294.

McCarthy, P. 2006. Checklist of the Lichens of Australia and its Island Territories. Australian Biological Resources Study, Canberra. Version 6. https://www.anbg.gov.au/abrs/lichenlist/introduction.html 
McDonald, T. R., Gaya, E. \& Lutzoni, F. 2013. Twenty-five cultures of lichenizing fungi available for experimental studies on symbiotic systems. Symbiosis 59: 165-171.

Meiklejohn, K. A., Damaso, N. \& Robertson, J. M. 2019. Assessment of BOLD and GenBank-Their accuracy and reliability for the identification of biological materials. PloS One 14(6): e021708.

Mercado-Díaz, J. A. 2009. Preliminary Checklist of Lichens and Lichenicolous Fungi of Puerto Rico (Version 1, July 2009). Published by the author, San Juan, Puerto Rico.

Mesta, A. R., Vinayaka, K. S., Darshini, S. M. \& Akarsh, S. 2016. Antimicrobial activity of Usnea ghattensis G. Awasthi and Usnea undulata Stirt. Journal of Chemical and Pharmaceutical Research 8: 83-88.

Mies, B. 1989. Vorarbeiten zu einer Flechtenflora der Kapverdischen Inseln. Untersuchungen zum Artenbestand und zur Verbreitung. Doctoral Thesis, University of Cologne.

Mies, B. 1993. Critical checklist of lichens and allied fungi of the Cape Verde Islands (lichenized Ascomycotina). Courier Forschungsinstitut Senckenberg 159: 153-174.

Millanes, A. M., Truong, C., Westberg, M., Diederich, P. \& Wedin, M. 2014. Host switching promotes diversity in host-specialized mycoparasitic fungi: uncoupled evolution in the Biatoropsis-Usnea system. Evolution 68: 1576-1593.

Miller, J. E. D. 2011. The Usnea rigida group in California and the Pacific Northwest. Bulletin of the California Lichen Society 18: 1-4.

Miller, M. A., Pfeiffer, W. \& Schwartz, T. 2010. Creating the CIPRES Science Gateway for inference of large phylogenetic trees. In: Proceedings of the Gateway Computing Environments Workshop $(G C E)$, pp. 1-8. New Orleans.

Moncada, B., Lücking, R. \& Suárez, A. 2014. Molecular phylogeny of the genus Sticta (lichenized Ascomycota: Lobariaceae) in Colombia. Fungal Diversity 64: 205-231.

Moncada, B., Mercado-Díaz, J. A., Magain, N., Hodkinson, B. P., Smith, C. W., Bungartz, F., Pérez-Pérez, R.-E., Gumboski, E., Sérusiaux, E., Lumbsch, H. T. \& Lücking, R. 2020a. Phylogenetic diversification of two geographically congruent species in the lichen genus Sticta (Ascomycota: Peltigeraceae): isolation by distance, ecology, or fragmentation? Journal of Biogeography (in press).

Moncada, B., Lücking, R. \& Lumbsch, H. T. 2020b. Rewriting the evolutionary history of the lichen genus Sticta (Ascomycota: Peltigeraceae subfam. Lobarioideae) in the Hawaiian islands. Plant and Fungal Systematics 65: 95-119.

Motyka, J. 1930. Materialy do flory porostów Slaska (Matériaux pour la connaissance des lichens de Silésie). Wydawncitwa Muzeum Söaskiego w Katowicach 3: 1-28.

Motyka, J. 1936. Lichenum Generis Usnea Studium Monographicum. Pars Systematica, Volumen Primum. Leopoli, Lublin.

Motyka, J. 1938. Lichenum Generis Usnea Studium Monographicum. Pars Systematica, Volumen Secundum. Leopoli, Lublin.

Myllys, L., Velmala, S., Lindgren, H., Glavich, D., Carlberg, T., LiSong, W. \& Goward, T. 2014. Taxonomic delimitation of the genera Bryoria and Sulcaria, with a new combination Sulcaria spiralifera introduced. The Lichenologist 46: 737-752.

Nadel, M. R. A. 2016. A Monograph of Usnea from São Tomé and Principe. Master's Thesis, San Francisco State University, USA.

Nascimbene, J. \& Tretiach, M. 2009. A critical evaluation of the Italian distribution of the rare macrolichen Usnea longissima Ach. Plant Biosystems 143: 14-19.

Nayaka, S. \& Upreti, D. K. 2005. Status of Lichen Diversity in Western Ghats, India. Sahyadri E-News, Western Ghats Biodiverstity Information System 16: 1-28.

Nilsson, R. H., Ryberg, M., Kristiansson, E., Abarenkov, K., Larsson, K. H. \& Kõljalg, U. 2006. Taxonomic reliability of DNA sequences in public sequence databases: a fungal perspective. PloS One 1(1): e59.
Nilsson, R. H., Kristiansson, E., Ryberg, M., Hallenberg, N. \& Larsson, K. H. 2008. Intraspecific ITS variability in the kingdom Fungi as expressed in the international sequence databases and its implications for molecular species identification. Evolutionary Bioinformatics 4: EBO-S653.

Nilsson, R. H., Taylor, A. F., Adams, R. I., Baschien, C., Bengtsson-Palme, J., Cangren, P., Coleine, C., Daniel, H. M., Glassman, S. I., Hirooka, Y. \& Irinyi, L. 2018. Taxonomic annotation of public fungal ITS sequences from the built environment - a report from an April 10-11, 2017 workshop (Aberdeen, UK). MycoKeys 28: 65-82.

Nilsson, R. H, Larsson, K.-H., Taylor, A. F. S., Bengtsson-Palme, J., Jeppesen, T. S., Schigel, D., Kennedy, P., Picard, K., Glöckner, F. O., Tedersoo, L., Saar, I., Kõljalg, U. \& Abarenkov, K. 2019. The UNITE database for molecular identification of fungi: handling dark taxa and parallel taxonomic classifications. Nucleic Acids Research 47(D1): D259-D264.

Nishanth, K. S., Sreerag, R. S., Deepa, I., Mohandas, C. \& Nambisan, B. 2015. Protocetraric acid: an excellent broad spectrum compound from the lichen Usnea albopunctata against medically important microbes. Natural Product Research 29: 574-577.

Noer, I. S., Ramlan, A., Subarnas, A. \& Sutedja, E. 2013. Karakterisasi dan Kekerabatan Janggot Kai (Usnea spp.) di Priangan [Characterization and relationship beard moss (Usnea spp.) at Priangan]. Indonesian Journal of Applied Sciences 3: 66-73.

Ohmura, Y. 2001. Taxonomic study of the genus Usnea (lichenized Ascomycetes) in Japan and Taiwan. Journal of the Hattori Botanical Laboratory 90: 1-96.

Ohmura, Y. 2002. Phylogenetic evaluation of infrageneric groups of the genus Usnea based on ITS regions in rDNA. Journal of the Hattori Botanical Laboratory 92: 231-243.

Ohmura, Y. 2008. Taxonomy and molecular phylogeny of Usnea rubicunda and U. rubrotincta (Parmeliaceae, lichenized Ascomycotina). Journal of Japanese Botany 83: 347-355.

Ohmura, Y. 2011. Notes on eight threatened species of lichens in Japan. Bulletin of the National Museum of Nature and Science Series B 37: 55-61.

Ohmura, Y. 2012. A synopsis of the lichen genus Usnea (Parmeliaceae, Ascomycota) in Taiwan. Memories of the National Science Museum Tokyo 48: 91-137.

Ohmura, Y. 2014. Usnea flavocardia (Parmeliaceae, lichenized Ascomycota) new to Asia. Bulletin of the National Museum of Natural Science, Ser. B, 40: 69-72.

Ohmura, Y. \& Clerc, P. 2019. Lectotypification of Usnea confusa (Parmeliaceae, Ascomycota). Bulletin of the National Museum of Natural Science, Ser. B, 45: 63-70.

Ohmura, Y. \& Kanda, H. 2004. Taxonomic status of section Neuropogon in the genus Usnea elucidated by morphological comparisons and ITS rDNA sequences. The Lichenologist 36: 217-225.

Ohmura, Y. \& Kashiwadani, H. 2018. Checklist of lichens and allied fungi of Japan. National Museum of Nature and Science Monographs 49: 1-140.

Ohmura, Y., Lin, C. K. \& Wang, P. H. 2010. Three sorediate species of the genus Usnea (Parmeliaceae, Ascomycota) new to Taiwan. Memoirs of the National Science Museum Tokyo 46: 69-76.

Ohmura, Y., Skirina, I. \& Skirin, F. 2017. Contribution to the knowledge of the genus Usnea (Parmeliaceae, Ascomycota) in southern Far East Russia. Bulletin of the National Museum of Natural Science, Ser. B, 43: 1-10.

Oldham, W. J., Hanson, S. K., Lavelle, K. B. \& Miller, J. L. 2016. Distribution of neptunium and plutonium in New Mexico lichen samples (Usnea arizonica) contaminated by atmospheric fallout. Journal of Radioanalytical and Nuclear Chemistry 307: 2079-2084.

Onuţ-Brännström, I., Tibell, L. \& Johannesson, H. 2017. A worldwide phylogeography of the whiteworm lichens Thamnolia reveals three lineages with distinct habitats and evolutionary histories. Ecology and Evolution 7: 3602-3615. 
Oran, S., Sahin, S., Sahinturk, P., Ozturk, S. \& Demir, C. 2016. Antioxidant and antimicrobial potential, and HPLC analysis of stictic and usnic acids of three Usnea species from Uludag mountain (Bursa, Turkey). Iranian Journal of Pharmaceutical Research 15: 527-535.

Orock, E. A., Leavitt, S. D., Fonge, B. A., St. Clair, L. L. \& Lumbsch, H. T. 2012. DNA-based identification of lichen-forming fungi: can publicly available sequence databases aid in lichen diversity inventories of Mount Cameroon (West Africa)? The Lichenologist 44: 833-839.

Osorio, H. S. 1992. Contribución a la flora liquénica del Uruguay. XXV. Líquenes publicados entre 1972 a 1991. Anales del Museo Nacional de Historia Natural de Montevideo, Ser. 2, 8: 43-70.

Otte, V. 2011. Usnea flavocardia found in Germany. Herzogia 24: $151-154$.

Paliya, B. S., Bajpai, R., Jadaun, V., Kumar, J., Kumar, S., Upreti, D. K., Singh, B. R., Nayaka, S., Joshi, Y. \& Singh, B. N. 2016. The genus Usnea: a potent phytomedicine with multifarious ethnobotany, phytochemistry and pharmacology. RSC Advances 6: 21672-21696.

Parnmen, S., Leavitt, S. D., Rangsiruji, A. \& Lumbsch, H. T. 2013. Identification of species in the Cladia aggregata group using DNA barcoding (Ascomycota: Lecanorales). Phytotaxa 115: 1-14.

Park, S. Y., Jang, S. H., Oh, S. O., Kim, J. A. \& Hur, J.-S. 2014. An easy, rapid, and cost-effective method for DNA extraction from various lichen taxa and specimens suitable for analysis of fungal and algal strains. Mycobiology 42: 311-316.

Park, C. H., Kim, K. M., Elvebakk, A., Kim, O. S., Jeong, G. \& Hong, S. G. 2015. Algal and fungal diversity in Antarctic lichens. Journal of Eukaryotic Microbiology 62: 196-205.

Pathak, A., Upreti, D. K. \& Dikshit, A. 2016. Antidermatophytic activity of the fruticose lichen Usnea orientalis. Medicines 3: 24.

Pavithra, G. M., Vinayaka, K. S., Rakesh, K. N., Junaid, S., Dileep, N., Prashit Kekuda, T. R, Siddiqua, S. \& Naik, A. S. 2013. Antimicrobial and antioxidant activities of a macrolichen Usnea pictoides G. Awasthi (Parmeliaceae). Journal of Applied Pharmaceutical Science 3: 154-160.

Peng, F., Ren, J. L., Xu, F., Su, Y. Q. \& Sun, R. C. 2010. Structural analysis of a fraction of polysaccharides from Usnea montis-fuji. Journal of Biobased Materials and Bioenergy 4: 12-15.

Pérez-Vargas, I., Padrón, C. H., Arroyo, R. \& Seriñá, E. 2010a. Usnea brasiliensis (Zahlbr.) Motyka (Parmeliaceae), a new amphi-Atlantic disjunct lichen species. The Bryologist 113: 308-312.

Pérez-Vargas, I., Hernández-Padrón, C., Arroyo, R. \& Seriñá, E. 2010 b. Usnea chaetophora, a lichen new to the Macaronesian Region. The Lichenologist 42: 347-351.

Pino-Bodas, R., Martín, M. P., Burgaz, A. R. \& Lumbsch, H. T. 2013. Species delimitation in Cladonia (Ascomycota): A challenge to the DNA barcoding philosophy. Molecular Ecology Resources 13: 1058-1068.

Pramyothin, P., Janthasoot, W., Pongnimitprasert, N., Phrukudom, S. \& Ruangrungsi, N. 2004. Hepatotoxic effect of $(+)$ usnic acid from Usnea siamensis Wainio in rats, isolated rat hepatocytes and isolated rat liver mitochondria. Journal of Ethnopharmacology 90: 381-387.

Rafat, A. 2014. Ecological and Biological Studies of New Zealand Lichens in the Genus Usnea. Doctoral Dissertation, Lincoln University, Lincoln.

Rafat, A., Ridgway, H. J., Cruickshank, R. H. \& Buckley, H. L. 2015. Isolation and co-culturing of symbionts in the genus Usnea. Symbiosis 66: 123-132.

Ramos, D. 2014. Lista de especies de líquenes y hongos liquenícolas del Perú. Glalia 6: 1-49.

Ramzaev, V. P., Barkovsky, A. N., Gromov, A. V., Ivanov, S. A. \& Kaduka, M. V. 2016. Temporal variations of ${ }^{7} \mathrm{Be},{ }^{40} \mathrm{~K},{ }^{134} \mathrm{Cs}$ and ${ }^{137} \mathrm{Cs}$ in epiphytic lichens (genus Usnea) at the Sakhalin and Kunashir islands after the Fukushima accident. Radiation Hygiene 9: 14-27.

Randlane, T., Tõrra, T., Saag, A. \& Saag, L. 2009. Key to European Usnea species. Bibliotheca Lichenologica 100: 419-462.
Ranft, H., Moncada, B., De Lange, P. J., Lumbsch, H. T. \& Lücking, R. 2018. The Sticta filix morphodeme (Ascomycota: Lobariaceae) in New Zealand, with the newly recognized species $S$. dendroides and $S$. menziesii: indicators of forest health in a threatened island biota? The Lichenologist 50: 185-210.

Ranković, B., Kosanić, M., Stanojković, T., Vasiljević, P. \& Manojlović, N. 2012. Biological activities of Toninia candida and Usnea barbata together with their norstictic acid and usnic acid constituents. International Journal of Molecular Sciences 13: 14707-14722.

Räsänen, V. 1931. Die Flechten Estlands. Mit einer Bestimmungstabelle der wichtigsten Nord- und Mitteleuropäischen Flechtenarten und Varietäten. I. Annales Academiae Scientiarum Fennicae, Ser. A, 34: $3-163$

Räsänen,V. 1936. Collationes ad lichenologiam Chilensem pertinentes. Revista Universitaria Santiago 21: 137-148.

Rehner, S. A. \& Uecker, F. A. 1994. Nuclear ribosomal internal transcribed spacer phylogeny and host diversity in the coelomycete Phomopsis. Canadian Journal of Botany 72: 1666-1674.

Rodriguez, J. M., Estrabou, C., Truong, C. \& Clerc, P. 2011a. The saxicolous species of the genus Usnea subgenus Usnea (Parmeliaceae) in Argentina and Uruguay. The Bryologist 114: 504-525.

Rodriguez, J. M., Estrabou, C., García, C. \& Farías, M. G. 2011b. High linear growth rate of the pendulous lichen Usnea angulata Ach. in Sierra Chaco Forest of Central Argentina. Sydowia 63: 105-112.

Rodríguez-Flakus, P., Kukwa, M., Etayo, J., Lücking, R., Meneses, R. I., Rivas Plata, E., Stanton, D., Truong, C., Vargas, R. \& Flakus, A. 2016. Preliminary Catalogue of Lichens and Lichenicolous Fungi from Bolivia. Version 1.5. https://bio.botany.pl/lichens-bolivia/ en, strona,catalogue, $5 . \mathrm{html}$

Rodríguez-R., E. F., Ramírez-O., Á. M., Alvítez-I., E., Pollack-V., L., Leiva-G., S. \& Aguirre-T., R. 2017. Catálogo de la liquenobiota de la región La Libertad, Perú. Arnaldoa 24: 497-522.

Rogers, R. W. \& Stevens, G. N. 1988. The Usnea baileyi complex (Parmeliaceae, lichenised ascomycetes) in Australia. Australian Systematic Botany 1: 355-361.

Rojas, J. L., Díaz-Santos, M. \& Valencia-Islas, N. A. 2015. Metabolites with antioxidant and photo-protective properties from Usnea roccellina Motyka, a lichen from Colombian Andes. UK Journal of Pharmaceutical and Biosciences 3: 18-26.

Rolstad, J., Ekman, S., Andersen, H. L. \& Rolstad, E. 2013. Genetic variation and reproductive mode in two epiphytic lichens of conservation concern: a transatlantic study of Evernia divaricata and Usnea longissima. Botany 91: 69-81.

Saag, L., Tõrra, T., Saag, A., Del-Prado, R. \& Randlane, T. 2011. Phylogenetic relations of European shrubby taxa of the genus Usnea. The Lichenologist 43: 427-444.

Saag, L., Mark, K., Saag, A. \& Randlane, T. 2014. Species delimitation in the lichenized fungal genus Vulpicida (Parmeliaceae, Ascomycota) using gene concatenation and coalescent-based species tree approaches. American Journal of Botany 101: 2169-2182.

Salgado, F., Albornoz, L., Cortéz, C., Stashenko, E., Urrea-Vallejo, K., Nagles, E., Galicia-Virviescas, C., Cornejo, A., Ardiles, A., Simirgiotis, M. \& García-Beltrán, O. 2018. Secondary metabolite profiling of species of the genus Usnea by UHPLC-ESI-OT-MS-MS. Molecules 23: 54.

Sanders, W. B. \& de los Ríos, A. 2012. Development of thallus axes in Usnea longissima (Parmeliaceae, Ascomycota), a fruticose lichen showing diffuse growth. American Journal of Botany 99: 998-1009.

Santiago, K. A. A., Sangvichien, E., Boonpragob, K. \& dela Cruz, T. E. E. 2013. Secondary metabolic profiling and antibacterial activities of different species of Usnea collected in Northern Philippines. Mycosphere 4: 267-280.

Santos, L. A., Aptroot, A., Lücking, R. \& Cáceres, M. E. S. 2019. High diversification in the Neoprotoparmelia multifera complex (Ascomycota, Parmeliaceae) in northeast Brazil revealed by DNA barcoding and phenotypical characters. The Bryologist 122: 539-552. 
Sasidharan, N. K., Sreekala, S. R., Lakshmanan, R., Jacob, J., Saraswathy Amma, D. K. B. N. \& Nambisan, B. 2014. Protolichesterinic acid: a prominent broad spectrum antimicrobial compound from the lichen Usnea albopunctata. International Journal of Antibiotics 2014: 302182

Schoch, C. L., Seifert, K. A., Huhndorf, S., Robert, V., Spouge, J. L., Levesque, C. A., Chen, W. \& Fungal Barcoding Consortium. 2012. Nuclear ribosomal internal transcribed spacer (ITS) region as a universal DNA barcode marker for Fungi. Proceedings of the National Academy of Sciences of the United States of America 109: 6241-6246.

Schoch, C. L., Robbertse, B., Robert, V., Vu, D., Cardinali, G., Irinyi, L., Meyer, W., Nilsson, R. H., Hughes, K., Miller, A. N., Kirk, P. M., Abarenkov, K., Aime, M. C., Ariyawansa, H. A., Bidartondo, M., Boekhout, T., Buyck, B., Cai, Q., Chen, J., Crespo, A., Crous, P. W., Damm, U., De Beer, Z. W., Dentinger, B. T., Divakar, P. K., Dueñas, M., Feau, N., Fliegerova, K., García, M. A., Ge, Z. W., Griffith, G. W., Groenewald, J. Z., Groenewald, M., Grube, M., Gryzenhout, M., Gueidan, C., Guo, L., Hambleton, S., Hamelin, R., Hansen, K., Hofstetter, V., Hong, S. B., Houbraken, J., Hyde, K. D., Inderbitzin, P., Johnston, P. R., Karunarathna, S. C., Kõljalg, U., Kovács, G. M., Kraichak, E., Krizsan, K., Kurtzman, C. P., Larsson, K. H., Leavitt, S., Letcher, P. M., Liimatainen, K., Liu, J. K., Lodge, D. J., Luangsa-ard, J. J., Lumbsch, H. T., Maharachchikumbura, S. S., Manamgoda, D., Martín, M. P., Minnis, A. M., Moncalvo, J. M., Mulè, G., Nakasone, K. K., Niskanen, T., Olariaga, I., Papp, T., Petkovits, T., Pino-Bodas, R., Powell, M. J., Raja, H. A., Redecker, D., Sarmiento-Ramirez, J. M., Seifert, K. A., Shrestha, B., Stenroos, S., Stielow, B., Suh, S. O., Tanaka, K., Tedersoo, L., Telleria, M. T., Udayanga, D., Untereiner, W. A., Diéguez Uribeondo, J., Subbarao, K. V., Vágvölgyi, C., Visagie, C., Voigt, K., Walker, D. M., Weir, B. S., Weiß, M., Wijayawardene, N. N., Wingfield, M. J., Xu, J. P., Yang, Z. L., Zhang, N., Zhuang, W. Y. \& Federhen, S. 2014. Finding needles in haystacks: linking scientific names, reference specimens and molecular data for Fungi. Database 2014: bau061.

Schoch, C. L., Aime, M. C., de Beer, W., Crous, P. W., Hyde, K. D., Penev, L., Seifert, K. A., Stadler, M., Zhang, N. \& Miller, A. N. 2017. Using standard keywords in publications to facilitate updates of new fungal taxonomic names. IMA Fungus 8: 70-73.

Schmull, M., Miadlikowska, J., Pelzer, M., Stocker-Wörgötter, E., Hofstetter, V., Fraker, E., Hodkinson, B. P., Reeb, V., Kukwa, M., Lumbsch, H. T. \& Kauff, F. 2011. Phylogenetic affiliations of members of the heterogeneous lichen-forming fungi of the genus Lecidea sensu Zahlbruckner (Lecanoromycetes, Ascomycota). Mycologia 103: 983-1003.

Schumm, F. \& Aptroot, A. 2010. Seychelles Lichen Guide. Beck, Süssen.

Seaward, M. R. D. \& Aptroot, A. 2006. A preliminary checklist of lichens for the Seychelles Group. Journal of the Hattori Botanical Laboratory 100: 765-781.

Seaward, M. R. D., Sipman, H. J. M., Schultz, M., Maassoumi, A. A., Anbaran, M. H. M. \& Sohrabi, M. 2004. A preliminary lichen checklist for Iran. Willdenowia 34: 543-576.

Seifert, K. A., Wingfield, B. D. \& Wingfield, M. J. 1995. A critique of DNA sequence analysis in the taxonomy of filamentous Ascomycetes and ascomycetous anamorphs. Canadian Journal of Botany 73(S1): 760-767.

Senkardesler, A. \& Clerc, P. 2017. Usnea pacificana P. Halonen (lichenized Ascomycetes) new to Eurasia. The Lichenologist 49: 99-101.

Seymour, F. A., Crittenden, P. D., Wirtz, N., Øvstedal, D. O., Dyer, P. S. \& Lumbsch, H. T. 2007. Phylogenetic and morphological analysis of Antarctic lichen-forming Usnea species in the group Neuropogon. Antarctic Science 19: 71-82.

Shen, Y. M., Hsieh, H. J., Yeh, R. Y. \& Hung, T. H. 2012. Five apothecium-producing lichenized fungi of the genus Usnea in Taiwan. Fungal Science 27: 31-44.

Shrestha, G. \& St Clair, L. L. 2013. Antimicrobial activity of extracts from two lichens Ramalina menziesii and Usnea lapponica. Bulletin of the California Lichen Society 20: 5-10.
Shrestha, G., Petersen, S. L. \& St Clair, L. L. 2012. Predicting the distribution of the air pollution sensitive lichen species Usnea hirta. The Lichenologist 44: 511-521.

Shukla, P., Upreti, D. K. \& Tewari, L. M. 2014. Lichen genus Usnea (Parmeliaceae, Ascomycota) in Uttarakhand, India. Current Research in Environmental \& Applied Mycology 4: 188-201.

Shukla, P., Upreti, D. K. \& Tewari, L. M. 2015. Secondary metabolite variability in lichen genus Usnea in India: A potential source for bioprospection. G-Journal of Environmental Science and Technology 2: 44-55.

Simon, A., Goffinet, B., Magain, N. \& Sérusiaux, E. 2018. High diversity, high insular endemism and recent origin in the lichen genus Sticta (lichenized Ascomycota, Peltigerales) in Madagascar and the Mascarenes. Molecular Phylogenetics and Evolution 122: 15-28.

Sinha, R., Abu-Ali, G., Vogtmann, E., Fodor, A. A., Ren, B., Amir, A., Schwager, E., Crabtree, J., Ma, S., Abnet, C. C., Knight, R., White, O. \& Huttenhower, C. 2017. Assessment of variation in microbial community amplicon sequencing by the Microbiome Quality Control (MBQC) project consortium. Nature Biotechnology 35: 1077.

Singh, K. P. \& Sinha, G. P. 2010. Indian Lichens: An Annotated Checklist. Botanical Survey of India, Ministry of Environment and Forests, Salt Lake City, Kolkata, India.

Singh, S., Khatoon, S., Joshi, Y., Prgyadeep, S., Upreti, D. K. \& Rawat, A. K. S. 2016. A validated HPTLC densitometric method for simultaneous determination of evernic and usnic acids in four Usnea species and comparison of their antioxidant potential. Journal of Chromatographic Science 54: 1670-1677.

Sipman, H. J. M. \& Aguirre-C., J. 2016. Líquenes. In: Bernal, R., Gradstein, S. R. \& Celis, M. (ed.s), Catálogo de Plantas y Líquenes de Colombia. Volumen 1, pp. 159-281. Universidad Nacional de Colombia, Facultad de Ciencias, Instituto de Ciencias Naturales, Bogotá.

Sipman, H. J. M., Diederich, P. \& Aptroot, A. 2013. New lichen records and a catalogue of lichens from Palawan Island, The Philippines. Philippine Journal of Science 142: 199-210.

Smith, C. W., Aptroot, A., Coppins, B. J., Fletcher, A., Gilbert, O. L., James, P. W. \& Wolseley, P. A. (ed.). 2009. The Lichens of Great Britain and Ireland. Natural History Museum \& British Lichen Society, London.

Smith, B. E., Johnston, M. K. \& Lücking, R. 2016. From GenBank to GBIF: Phylogeny-based predictive niche modeling tests accuracy of taxonomic identifications in large occurrence data repositories. PLoS One 11(3): e0151232.

Soler, R., Martínez Pastur, G., Lencinas, M. V. \& Rosenfeld, M. 2014. Variable retention management influences biomass of Misodendrum and Usnea in Nothofagus pumilio southern Patagonian forests. New Zealand Journal of Botany 52: 224-235.

Šoun, J., Vondrák, J. \& Bouda, F. 2015. Vzácné a málo známé druhy lišejníků Třebíčska a okolí [Rare and little known species in the Třebíč region and surroundings]. Bryonora 56: 1-23.

Spatafora, J. W., Sung, G.-H., Johnson, D., O’Rourke, B., Serdani, M., Spotts, R., Lutzoni, F., Hofstetter, V., Miadlikowska, J., Reeb, V., Gueidan, C., Fraker, E., Lumbsch, H. T., Lücking, R., Schmitt, I., Hosaka, K., Aptroot, A., Roux, C., Miller, A., Geiser, D., Hafellner, J., Hestmark, G., Arnold, A. E., Büdel, B., Rauhut, A., Hewitt, D., Untereiner, W. A., Cole, M. S., Scheidegger, C., Schultz, M., Sipman, H. J. M. \& Schoch, C. 2006. A five-gene phylogeny of Pezizomycotina. Mycologia 98: 1018-1028.

Spielmann, A. A. 2006. Checklist of lichens and lichenicolous fungi of Rio Grande do Sul (Brazil). Caderno de Pesquisa Série Biologia 18: 7-125.

Spribille, T., Goffinet, B., Klug, B., Muggia, L., Obermayer, W. \& Mayrhofer, H. 2011. Molecular support for the recognition of the Mycoblastus fucatus group as the new genus Violella (Tephromelataceae, Lecanorales). The Lichenologist 43: 445-466. 
Stamatakis, A. 2014. RAxML version 8: a tool for phylogenetic analysis and post-analysis of large phylogenies. Bioinformatics 30: 1312-1313.

Steiner, J. 1897. Flechten aus Britisch-Ostafrika. Sitzungsberichte der kaiserlichen Akademie der Wissenschaften Wien, Mathematish-Naturwissenschaftliche Classe 106, Abt. I: 207-234.

Stevens, G. N. 1990. Usnea himantodes Stirton and its synonyms. The Lichenologist 22: 409-412.

Stevens, G. N. 1999. A revision of the lichen family Usneaceae in Australia. Bibliotheca Lichenologica 72: 1-128.

Stielow, J. B., Levesque, C. A., Seifert, K. A., Meyer, W., Iriny, L., Smits, D., Renfurm, R., Verkley, G. J. M., Groenewald, M., Chaduli, D., Lomascolo, A., Welti, S., Lesage-Meessen, L., Favel, A., Al-Hatmi, A. M. S., Damm, U., Yilmaz, N., Houbraken, J., Lombard, L., Quaedvlieg, W., Binder, M., Vaas, L. A. I., Vu, D., Yurkov, A. M., Begerow, D., Roehl, O., Guerreiro, M., Fonseca, A., Samerpitak, K., van Diepeningen, A. D., Dolatabadi, S., Moreno, L. F., Casaregola, S., Mallet, S., Jacques, N., Roscini, L., Egidi, E., Bizet, C., Garcia-Hermoso, D., Martín, M. P., Deng, S., Groenewald, J. Z., Boekhout, T., de Beer, Z. W., Barnes, I., Duong, T. A., Wingfield, M. J., de Hoog, G. S., Crous, P. W., Lewis, C. T., Hambleton, S., Moussa, T. A. A., Al-Zahrani, H. S., Almaghrabi, O. A., Louis-Seize, G., Assabgui, R., McCormick, W., Omer, G., Dukik, K., Cardinali, G., Eberhardt, U., de Vries, M. \& Robert, V. 2015. One fungus, which genes? Development and assessment of universal primers for potential secondary fungal DNA barcodes. Persoonia 35: 242-263.

Stirton, J. 1881. On the genus Usnea and a new genus allied to it. Scottish Naturalist 6: 100-107.

Stirton, J. 1883. Notes on Usnea. Scottish Naturalist 7: 74-79.

Storaunet, K. O., Rolstad, J. \& Rolstad, E. 2014. Effects of logging on the threatened epiphytic lichen Usnea longissima: an experimental approach. Silva Fennica 48: 949.

Strigina, L. I. \& Sviridov, V. N. 1978. $5 \alpha, 8 \alpha$-epidioxy-5 $\alpha$-ergosta-6, 22-diene-3 $\beta$-ol from Usnea annulata and Dactylina arctica. Phytochemistry 17: 327-327.

Suetina, Y. G. \& Glotov, N. V. 2010. Ontogeny and morphogenesis of the fruticose lichen Usnea florida (L.) Weber ex F.H. Wigg. Russian Journal of Developmental Biology 41: 24-31.

Sultana, N. \& Afolayan, A. J. 2011. A new depsidone and antibacterial activities of compounds from Usnea undulata Stirton. Journal of Asian Natural Products Research 13: 1158-1164.

Swinscow, T. D. V. \& Krog, H. 1974. Usnea subgenus Eumitria in East Africa. Norwegian Journal of Botany 21: 165-185.

Swinscow, T. D. V. \& Krog, H. 1975. The Usnea undulata aggregate in East Africa. The Lichenologist 7: 121-138.

Swinscow, T. D. V. \& Krog, H. 1976a. The Usnea articulata aggregate in East Africa. Norwegian Journal of Botany 23: 261-268.

Swinscow, T. D. V. \& Krog, H. 1976b. The Usnea bornmuelleri aggregate in East Africa. Norwegian Journal of Botany 23: 23-31.

Swinscow, T. D. V. \& Krog, H. 1978. Pendulous species of Usnea in East Africa. Norwegian Journal of Botany 25: 221-241.

Swinscow, T. D. V. \& Krog, H. 1979. The fruticose species of Usnea subgenus Usnea in East Africa. The Lichenologist 11: 207-252.

Swinscow, T. D. V. \& Krog, H. 1986. Usnea antiqua sp. nov. described from Tanzania. The Lichenologist 18: 293-295.

Swinscow, T. D. V. \& Krog, H. 1988. Macrolichens of East Africa. British Museum (Natural History), London.

Tavares, C. N. 1964. Lichenes Lusitaniae Selecti Exsiccati, Editi ab Instituto Botanico Universitatis Olisiponesis. Fasciculus VIII. Junii 1964. Faculdade de Ciencias, Instituto Botanico, Lisboa.

Tavares, I. I. 1987. The taxa of Usnea (Lichenes) described by Michaux from eastern North America. Mycotaxon 30: 39-68.

Taylor, T. 1847. New lichens, principally from the herbarium of Sir William J. Hooker. London Journal of Botany 4: 148-197.
Temu, S. G., Clerc, P., Tibell, L., Tibuhwa, D. D., Tibell, S. 2019 Phylogeny of the subgenus Eumitria in Tanzania. Mycology 10: 250-260.

Thell, A., Stenroos, S., Feuerer, T., Kärnefelt, I., Myllys, L. \& Hyvönen, J. 2002. Phylogeny of cetrarioid lichens (Parmeliaceae) inferred from ITS and b-tubulin sequences, morphology, anatomy and secondary chemistry. Mycological Progress 1: 335-354.

Thell, A., Feuerer, T., Kärnefelt, I., Myllys, L. \& Stenroos, S. 2004 Monophyletic groups within the Parmeliaceae identified by ITS rDNA, $\beta$-tubulin and GAPDH sequences. Mycological Progress 3: $297-314$.

Thell, A., Crespo, A., Divakar, P. K., Kärnefelt, I., Leavitt, S. D., Lumbsch, H. T. \& Seaward, M R D. 2012. A review of the lichen family Parmeliaceae - History, phylogeny and current taxonomy. Nordic Journal of Botany 30: 641-664.

Thippeswamy, B., Naveenkumar, K. J., Bodharthi, J. G. \& Shivaprasad, S. R. 2011. Antimicrobial activity of ethanolic extract of Usnea longissima. Journal of Experimental Sciences 2: 1-3.

Timbreza, L. P., De los Reyes, J. L., Flores, C. H., Perez, R. J., Stockel, M. A. \& Santiago, K. A. 2017. Antibacterial activities of the lichen Ramalina and Usnea collected from Mt. Banoi, Batangas and Dahilayan, Bukidnon, against multi-drug resistant (MDR) bacteria. Östereichische Zeitschrift für Pilzkunde 26: 27-42.

Tisserat, N. A., Hulbert, S. H. \& Sauer, K. M. 1994. Selective amplification of rDNA internal transcribed spacer regions to detect Ophiosphaerella korrae and O. herpotricha. Phytopathology 84: 478-482.

Tõrra, T., Cornejo, C., Cheenacharoen, S., Dal Grande, F., Marmor, L. \& Scheidegger, C. 2014. Characterization of fungus-specific microsatellite markers in the lichen fungus Usnea subfloridana (Parmeliaceae). Applications in Plant Sciences 2: 1400034

Tozatti, M. G., Ferreira, D. S., Flauzino, L. G. B., Silva Moraes, T., Martins, C. H., Groppo, M., Silva, M. L. A. E., Januario, A. H., Pauletti, P. M. \& Cunha, W. R. 2016. Activity of the lichen Usnea steineri and its major metabolites against gram-positive, multidrug-resistant bacteria. Natural Product Communications 11: 1934578X1601100419.

Truong, C. 2012. Systematics of the Lichen Genus Usnea in Tropical South America. Doctoral Dissertation, University of Geneva.

Truong, C. \& Clerc, P. 2012. The lichen genus Usnea (Parmeliaceae) in tropical South America: species with a pigmented medulla, reacting C+ yellow. The Lichenologist 44: 625-637.

Truong, C. \& Clerc, P. 2013. Eumitrioid Usnea species (Parmeliaceae, lichenized Ascomycota) in tropical South America and the Galapagos. The Lichenologist 45: 383-395.

Truong, C. \& Clerc, P. 2016. New species and new records in the genus Usnea (Parmeliaceae, lichenized Ascomycota) from tropical South America. The Lichenologist 48: 71-93.

Truong, C., Bungartz, F. \& Clerc, P. 2011. The lichen genus Usnea (Parmeliaceae) in the tropical Andes and the Galapagos: species with a red-orange cortical or subcortical pigmentation. The Bryologist 114: 477-503.

Truong, C., Divakar, P. K., Yahr, R., Crespo, A. \& Clerc, P. 2013a. Testing the use of ITS rDNA and protein-coding genes in the generic and species delimitation of the lichen genus Usnea (Parmeliaceae, Ascomycota). Molecular Phylogenetics and Evolution 68: 357-372.

Truong, C., Rodridguez, J. M. \& Clerc, P. 2013b. Pendulous Usnea species (Parmeliaceae, lichenized Ascomycota) in tropical South America and the Galapagos. The Lichenologist 45: 505-542.

Truong, T. L., Nga, V. T., Huy, D. T., Chi, H. B. \& Phung, N. K. 2014. A new depside from Usnea aciculifera growing in Vietnam. Natural Product Communications 9: 1934578X1400900831.

Tsurykau, A. 2018. A provisional checklist of the lichens of Belarus. Opuscula Philolichenum 17: 374-479.

Upreti, D. K., Tiwari, P., Shukla, P. \& Dwivedi, A. 2012. Natural thalli and cultured mycobiont of Usnea ghattensis G. Awasthi - A potential source of purple dye yielding lichen from India. Indian Journal of Natural Products and Resources 3: 489-492. 
Urbanavichus, G. 2010. A Checklist of the Lichen Flora of Russia. Nauka, St. Petersburg.

Vainio, E. A. 1890. Etude sur la classification et la morphologie des lichens du Brésil, I. Acta Societatis pro Fauna et Flora Fennica 7: V-XXIX, 1-247.

van den Boom, P. P. \& Ertz, D. 2012. Lichens and lichenicolous fungi from El Hierro (Canary Islands), a survey, including five new species. Cryptogamie, Mycologie 33: 59-97.

van den Boom, P. P., Clerc, P. \& Ertz, D. 2015. New records of lichens and lichenicolous fungi from La Gomera (Canary Islands, Spain), including the new species: Usnea boomiana P. Clerc. Candollea 70: $165-177$.

Van Nguyen, K. \& Duong, H. T. 2018. Extraction, isolation and characterization of depsidones from Usnea baileyi (Stirt.) Zahlbr collected from tree barks in Tam Bo mountain of Di Linh, Lam Dong province, Viet Nam. Science and Technology Development Journal 21: 24-31.

Van Nguyen, K., Duong, T. H., Nguyen, K. P. P., Sangvichien, E., Wonganan, P. \& Chavasiri, W. 2018. Chemical constituents of the lichen Usnea baileyi (Stirt.) Zahlbr. Tetrahedron Letters 59: 1348-1351.

Vareschi, V. 2001. El género Usnea en Venezuela. Boletín de la Academia de Ciencias Físicas Matemáticas Y Naturales de Venezuela 61: 9-63.

Vu, D., Groenewald, M., Szöke, S., Cardinali, G., Eberhardt, U., Stielow, B., de Vries, M., Verkleij, G. J. M., Crous, P. W., Boekhout, T. \& Robert, V. 2016. DNA barcoding analysis of more than 9000 yeast isolates contributes to quantitative thresholds for yeast species and genera delimitation. Studies in Mycology 85: 91-105.

Vu, D., Groenewald, M., de Vries, M., Gehrmann, T., Stielow, B., Eberhardt, U., Al-Hatmi, A., Groenewald, J. Z., Cardinali, G., Houbraken, J., Boekhout, T., Crous, P. W., Robert, V. \& Verkley, G. J. M. 2019. Large-scale generation and analysis of filamentous fungal DNA barcodes boosts coverage for kingdom fungi and reveals thresholds for fungal species and higher taxon delimitation. Studies in Mycology 92: 135-154.

Walker, F J. 1985. The lichen genus Usnea subgenus Neuropogon. Bulletin of the British Museum (Natural History), Botany Series 13: 1-130.

Wang, Y., Kim, J. A., Cheong, Y. H., Joshi, Y., Koh, Y. J. \& Hur, J. S. 2011. Isolation and characterization of a reducing polyketide synthase gene from the lichen-forming fungus Usnea longissima. The Journal of Microbiology 49: 473-480.

Wedin, M., Döring, H. \& Mattsson, J. E. 1999. A multi-gene study of the phylogenetic relationships of the Parmeliaceae. Mycological Research 103: 1185-1192.

White, T. J., Bruns, T., Lee, S. \& Taylor, J. 1990. Amplification and direct sequencing of ribosomal RNA genes and the internal transcribed spacer in fungi. In: Innis, M. A., Gelfand, D. H., Sninsky, J. J. \& White, T. H. (eds), PCR-Protocols and Applications - A Laboratory Manual, pp. 315-322. Academic Press, New York.

Widhelm, T. J., Grewe, F., Huang, J. P., Mercado-Díaz, J. A., Goffinet, B., Lücking, R., Moncada, B., Mason-Gamer, R. \& Lumbsch, H. T.
2019. Multiple historical processes obscure phylogenetic relationships in a taxonomically difficult group (Lobariaceae, Ascomycota). Scientific Reports 9: 1-16.

Wirtz, N., Printzen, C., Sancho, L. G. \& Lumbsch, T. H. 2006. The phylogeny and classification of Neuropogon and Usnea (Parmeliaceae, Ascomycota) revisited. Taxon 55: 367-376.

Wirtz, N., Printzen, C. \& Lumbsch, H. T. 2008. The delimitation of Antarctic and bipolar species of neuropogonoid Usnea (Ascomycota, Lecanorales): a cohesion approach of species recognition for the Usnea perpusilla complex. Mycological Research 112: 472-484.

Wirtz, N., Printzen, C. \& Lumbsch, H. T. 2012. Using haplotype networks, estimation of gene flow and phenotypic characters to understand species delimitation in fungi of a predominantly Antarctic Usnea group (Ascomycota, Parmeliaceae). Organisms Diversity \& Evolution 12: 17-37.

Woo, J.-J., Lücking, R., Oh, S.-Y., Jeun, Y.-C. \& Hur, J.-S. 2020. Two new foliicolous species of Strigula (Strigulaceae, Strigulales) in Korea offer insight in phorophyte-dependent variation of thallus morphology. Phytotaxa 443: 1-2.

Xu, H. B., Yang, T. H., Xie, P., Liu, S.J., Liang, Y. N., Zhang, Y., Song, Z. X. \& Tang, Z. S. 2018. Phaeophytin analogues from the medicinal lichen Usnea diffracta. Natural Product Research 32: 1088-1094.

Yildirim, E., Aslan, A., Emsen, B., Cakir, A. \& Ercisli, S. 2012. Insecticidal effect of Usnea longissima (Parmeliaceae) extract against Sitophilus granarius (Coleoptera: Curculionidae). International Journal of Agriculture and Biology 14: 303-306.

Yu, X., Guo, Q., Su, G., Yang, A., Hu, Z., Qu, C., Wan, Z., Li, R., Tu, P. \& Chai, X. 2016. Usnic acid derivatives with cytotoxic and antifungal activities from the lichen Usnea longissima. Journal of Natural Products 79: 1373-1380.

Zahlbruckner, A. 1905. Lichenes, a cl. Damazio in Brasilia lecti. II. Bulletin L'Herbier Boissier, Ser. 2, 5: 539-543.

Zahlbruckner, A. 1908. Beiträge zur Flechtenflora Brasiliens. Bulletin L'Herbier Boissier, Ser. 2, 8: 459-468.

Zahlbruckner, A. 1933. Flechten der Insel Formosa (Fortsetzung und Schluß). Feddes Repertorium 33: 22-68.

Zelada, B. R. \& Pastor de Abram, A. 2012. Estudio fitoquímico de Usnea durietzii Mot. (Usneaceae). Revista de la Sociedad Química del Perú 78: 264-276.

Zhao, Z., Liu, H., Luo, Y., Zhou, S., An, L., Wang, C., Jin, Q., Zhou, M. \& Xu, J. R. 2014. Molecular evolution and functional divergence of tubulin superfamily in the fungal tree of life. Scientific Reports 4: 6746.

Zoller, S., Lutzoni, F. \& Scheidegger, C. 1999. Genetic variation within and among populations of the threatened lichen Lobaria pulmonaria in Switzerland and implications for its conservation. Molecular Ecology 8: 2049-2059.

Zvěřina, O., Láska, K., Červenka, R., Kuta, J., Coufalík, P. \& Komárek, J. 2014. Analysis of mercury and other heavy metals accumulated in lichen Usnea antarctica from James Ross Island, Antarctica. Environmental Monitoring and Assessment 186: 9089-9100. 